\title{
Infrared Problem vs Gauge Choice: Scattering of Classical Dirac Field
}

\author{
Andrzej Herdegen 10
}

\begin{abstract}
We consider the Dirac equation for the classical spinor field placed in an external, time-dependent electromagnetic field of the form typical for scattering settings: $F=F^{\mathrm{ret}}+F^{\mathrm{in}}=F^{\mathrm{adv}}+F^{\mathrm{out}}$, where the current producing $F^{\text {ret/adv }}$ has past and future asymptotes homogeneous of degree -3 , and the free fields $F^{\text {in/out }}$ are radiation fields produced by currents with similar asymptotic behavior. We show the existence of the electromagnetic gauges in which the particle has 'in' and 'out' asymptotic states approaching free field states, with no long-time corrections of the free dynamics. Using a special Cauchy foliation of the spacetime, we show in this context the existence and asymptotic completeness of the wave operators. Moreover, we define a special 'evolution picture' in which the free evolution operator has well-defined limits for $t \rightarrow \pm \infty$; thus the scattering wave operators do not need the free evolution counteraction.
\end{abstract}

Mathematics Subject Classification. Primary 81U99; Secondary 81V10.

\section{Introduction}

This article is a further step in a program of investigation of the infrared problems in electrodynamics. Among them, the long-time asymptotic behavior of the charged matter fields is one of the key issues. Such questions as understanding what is a charged particle, or how to define scattering operator in quantum electrodynamics, are correlated to this problem.

It is well known that the long-time asymptotics poses problems in theories with long-range interactions. Standard way to deal with that is to modify the asymptotic dynamics of charged particles or fields, by Dollard or similar methods, augmented by some 'dressing' of charged particles. The cost is the 
loss of a clear interpretation of the asymptotic dynamics. Moreover, in physically realistic quantum field theory models this procedure has not resulted, up to now, in a non-perturbational understanding of the issue. ${ }^{1}$

In a series of articles I have put forward the idea, that the long-time asymptotics problem may be relieved by an appropriate choice of gauge of the electromagnetic potential. In a recent article [11] a Schrödinger (nonrelativistic) particle was considered in a time-dependent electromagnetic field, of the form typical for scattering situations. It was found that an appropriate choice of gauge allows the existence of the asymptotic dynamics, with no need for dressing. ${ }^{2}$ We also refer the reader to this article for more extensive description of the context and our motivation.

Here we implement the idea in the case of the classical Dirac field evolving in the external electromagnetic field. The system is not fully self-interacting, but the form of the external electromagnetic field mimics the expected properties of this field in fully interacting system. We consider the evolution of the Dirac field as a unitary evolution in a Hilbert space. However, similarly as in the previously considered nonrelativistic case, the evolution does not take place in standard time, over flat pure space sheets. It turns out, that for our purpose it is convenient to consider a Cauchy foliation supplied by constant $\tau$ surfaces, where $(\tau, \mathbf{z})$ form the coordinate system ${ }^{3}$ defined by:

$$
x^{0}=\tau\left(|\mathbf{z}|^{2}+1\right)^{\frac{1}{2}}, \quad \mathbf{x}=\left(\tau^{2}+1\right)^{\frac{1}{2}} \mathbf{z} .
$$

This poses technical complication even at the free Dirac equation level, as the unitary evolution is not a unitary one-parameter group (Hamiltonian depends

\footnotetext{
${ }^{1}$ For (generalized) Dollard methods, see the monograph [2]. Recent examples of the use of 'dressing' in construction of simplified quantum field models include $[5,13]$. A recent attempt at a precise implementation of the Dollard idea in general quantum field theory (in the form proposed much earlier in rather imprecise terms by Kulish and Faddeev) may be found in [4].

${ }^{2}$ What remains an open question for this system is the asymptotic completeness. In the article, we expressed the view that the clash between the symmetry groups of the two parts of the system: Lorentz for the Maxwell and Galilean for the Schrödinger equations, might make the issue more problematic. Our present discussion seems to confirm this.

${ }^{3}$ I have first proposed the use of these coordinates in the present context in 1999, as a natural extension of the hyperbolic foliation of the inside of the lightcone, to a Cauchy foliation of the whole spacetime. The evolution of the Dirac field over the hyperbolic foliation, and its large hyperbolic time asymptotics, was analyzed in [8] in terms of a Fourier-like transformation, which transformed this evolution to a unitary evolution in the Hilbert space of spinors on the hyperboloid of four velocities. The existence of the wave operators for the Dirac field in external electromagnetic field was established (in appropriate gauge, see below), but no results on asymptotic completeness were obtained. The idea now was to formulate the evolution over the foliation (1) in similar Fourier terms. Piotr Marecki, my student at that time, carried out in his MSc Thesis [12] calculations of this program for the free Dirac field (scattering was only briefly mentioned in this thesis). This method has its assets, but it is inconvenient for the analysis of many further questions like selfadjointness of the generators, the analysis of the domain of validity of the Dirac equation in the differential form, or asymptotic completeness of the interacting case, the questions that have not been considered. Here we use another method, which could be developed much further, and enabled the solution of all these questions.
} 
on $\tau) .{ }^{4}$ This is to be contrasted with the nonrelativistic case considered earlier, where the Niederer transformation introducing suitable coordinates leads from the free particle Hamiltonian to that of the harmonic oscillator (a kind of beautiful miracle). Nevertheless, the Schrödinger operator case becomes steeply difficult when the potential with space part is introduced, as then the term $\mathbf{A} \cdot \mathbf{p}$ prevents the application of the Dyson series method. To deal with that case, we have applied the Kato theorem. Here, for the free Dirac equation we proceed differently, and our method may be of independent interest. The addition of the interaction with the electromagnetic field may be then treated, in contrast to the nonrelativistic Schrödinger case, by a variation of the Dyson method combined with the relativistic causality. For this system we show that an appropriate choice of gauge removes the asymptotic problem. We show the existence and asymptotic completeness of the wave operators, with no need for any modification of the asymptotic free Dirac evolution. ${ }^{5}$

This result will show that the choice of a gauge $\mathcal{A}(x)$ in this classical field setting has a decisive importance for the asymptotic identification of the incoming/outgoing charged fields. The main qualitative feature of gauges in this appropriate class is that the product $x \cdot \mathcal{A}(x)$ vanishes in timelike directions, see remarks in Sect. 8 (a property already identified in [8]). We would like to stress that the theory is formulated from the outset in such chosen gauge. Whether such formulation may be carried over to the quantum field theory is a subject for future research. ${ }^{6}$ This prospective investigation should also find contact with the asymptotic algebra of fields in quantum electrodynamics postulated by the author [9] (see also [10]).

Here let us only mention that the use of a gauge in the class anticipated above could lead to some broadening of the scope of external classical, time-dependent electromagnetic fields for which the scattering operator for the classical Dirac field may be lifted to the case of the respective quantum field. As is well known, the mixing of electrons/positrons leads to rather severe

\footnotetext{
4 One should also mention that the use of more general, than the flat equal time hypersurfaces, places our problem as a special case of the problem of hyperbolic equations on Lorentzian manifolds, a question intensively studied in the past, see a recent monograph [1]. However, our more specific system allows us to apply more specific Hilbert space methods, and obtain stronger results.

${ }^{5}$ The Cauchy problem and the asymptotic completeness of the interacting Maxwell-Dirac system were considered by Flato et al. in [7]. However, their analysis needs strong smoothness and smallness assumptions, the latter not under well-determined control, and uses methods rather not well suited for an application in quantum case ('nonlinear representation of the Poincaré group'). Also, the authors modify the asymptotic dynamics by a variation of the Dollard method. Our aims are different, as explained above.

${ }^{6}$ We postpone to such prospective publication a comparison with the existing discussions of the relevance of gauge choice in QED. Here counts the idea, originated by Dirac [3], of an $a$ posteriori transformation to a 'gauge-independent gauge,' developed by many authors, most exhaustively by Steinmann, see his monograph [18], Chapter 12. Also, the expectation (not shared by everyone) that formulations of QED in differing gauges need not be equivalent received a recent support from a mathematical analysis of a simplified model [6] (where literature account may also be found).
} 
restrictions in this respect ${ }^{7}$ (see, e.g., the monograph [17], Sections 2.4 and 2.5). Precisely what gain would be possible is an open question.

The outline of the article is as follows. In Sect. 2 we formulate the Dirac evolution as a unitary evolution, with time-dependent self-adjoint generators, over a rather general family of Cauchy surfaces. Section 3 gives the formulation of the external field problem with the Dyson series method, but with the important use of the relativistic causality. In both free and interacting case the self-adjointness is achieved with the use of the commutator theorem (see Appendix B) and the harmonic oscillator Hamiltonian. In Sect. 4 we specify our choice of coordinates to those mentioned above and transform the evolution to a new 'picture.' In this picture the free Dirac evolution on our foliation has well-defined limits as unitary operators. Section 5 specifies the general external problem to the electromagnetic case and discusses the gauge transformation. Section 6 contains our central results formulated for a wide class of electromagnetic fields: the existence and asymptotic completeness of the wave operators. Section 7 gives a theorem showing that the electromagnetic fields typical for scattering contexts described above admit potentials in gauges satisfying the demands of the main theorems of Sect. 6. Section 8 offers some remarks on the implications of our results and on further physically motivated restriction in the class of the obtained electromagnetic gauges.

Large parts of the material are shifted to Appendix. In Appendix A we discuss a spinor transformation needed in Sect. 2. Appendix B describes our method to deal with a class of time-dependent Hamiltonians. A lemma needed for the application of this method to the system considered here is discussed in Appendix C. In Appendix D we gather geometrical facts and relations in our special coordinate system. Appendices E and F recapitulate some properties of the solutions of the free Dirac and wave equations, respectively. Appendix G contains some estimates of the decay of the advanced and retarded solutions of the inhomogeneous wave equation and their differences (radiation fields). The results of Appendices $\mathrm{E}-\mathrm{G}$ are applied next in Appendix $\mathrm{H}$ to the case of the electromagnetic fields typical for scattering contexts. Finally, the necessary decay properties of the special gauge introduced in Sect. 7 are obtained in Appendix I.

\section{Free Dirac Evolution}

Throughout the article we set $\hbar=1, c=1$. We choose a reference point, and then the flat spacetime is identified with the Minkowski vector space. Let $m$

\footnotetext{
${ }^{7}$ This is a standard example of the fact that classical external interaction problems for quantum fields create problems of their own, which are not expected to propagate into full closed quantum theory. An even more restrictive question in the case of the quantum Dirac field in an external classical field is whether a unitary evolution operator exists for this system. As shown in [16] (for standard evolution over the flat foliation of the spacetime), this may be possible only if the magnetic field vanishes. This brings to sharp light a rather restricted physical relevance of such questions and models (note that this condition is not even inertial observer-independent; anyway, this problem is not related to infrared questions).
} 
be the mass parameter in the free Dirac equation. To simplify notation, we rescale Minkowski vectors by multiplying them by $m$, and denote the resulting space by $M$, and its dimensionless vectors by $x^{a}$. The flat (covariant) derivative in the rescaled Minkowski space is denoted by $\nabla_{a}$. Also, the electromagnetic interaction to appear later will be introduced by the interaction term $\mathcal{A}_{a} \bar{\psi} \gamma^{a} \psi$, so to recover the physical units and quantities one should replace $\mathcal{A} \rightarrow(e / m) \mathcal{A}$, with $e$ the elementary charge.

Let $\tau: M \mapsto \mathbb{R}$ be a smooth surjective function such that the hypersurfaces $\Sigma_{\tau}$ of constant $\tau$ form a Cauchy foliation of the Minkowski spacetime, with $\tau$ increasing into the future. Consider the Dirac equation, which we write in the form

$$
\left(\frac{1}{2}\left[\gamma^{a}, i \nabla_{a}\right]_{+}-1\right) \psi=0
$$

in our dimensionless coordinates, where $\gamma^{a}$ are the Dirac matrices and $[., .]_{+}$ symbolizes the anticommutator. As is well known, the Cauchy problem for this equation with the initial data $\left.\psi\right|_{\Sigma_{\tau_{0}}}=f$ is explicitly solved by the formula

$$
\psi(x)=i^{-1} \int_{\Sigma_{\tau_{0}}} S(x-y) \gamma^{a} f(y) \mathrm{d} \sigma_{a}(y)
$$

where $\mathrm{d} \sigma_{a}$ is the dual integration element on $\Sigma_{\tau_{0}}$ and $S(x)$ is the standard Green function of the free Dirac field, in the dimensionless coordinates

$$
S(x)=(i \gamma \cdot \nabla+1) D_{1}(x), \quad D_{1}(x)=\frac{i}{(2 \pi)^{3}} \int \operatorname{sgn}\left(v^{0}\right) \delta\left(v^{2}-1\right) e^{-i v \cdot x} \mathrm{~d} v .
$$

If $f$ is a smooth bi-spinor function on $\Sigma_{\tau_{0}}$, with a compact support, then $\psi(x)$ is a smooth function in $M$, with compact support on each Cauchy surface $\Sigma_{\tau}$. Therefore, we obtain a bijective evolution mapping between the spaces of smooth, compactly supported bi-spinor functions on our family of Cauchy surfaces. Moreover, if on each $\Sigma_{\tau}$ one defines the scalar product

$$
\left(\psi_{1}, \psi_{2}\right)_{\tau}=\int_{\Sigma_{\tau}} \overline{\psi_{1}} \gamma^{a} \psi_{2} \mathrm{~d} \sigma_{a},
$$

then the evolution is isometric.

Let now $\left(\zeta^{\mu}\right)=\left(\zeta^{0}, \zeta^{i}\right)=\left(\tau, z^{i}\right) \equiv(\tau, \mathbf{z}) \in \mathbb{R}^{4}(i=1,2,3)$, with $\tau$ described above, be a smooth curvilinear coordinate system, mapping $M$ diffeomorphically onto $\mathbb{R}^{4}$. Denote by $\eta_{a b}$ and $C^{a \ldots}{ }_{b} \ldots$ the Minkowski spacetime metric tensor, and any other tensor, respectively. Then the geometrical components in the coordinate system $\left(\xi^{\mu}\right)$ will be denoted by

$$
\begin{aligned}
& \partial_{\mu}=\frac{\partial}{\partial \zeta^{\mu}}=\frac{\partial x^{a}}{\partial \zeta^{\mu}} \nabla_{a}, \quad \partial_{\tau}=\frac{\partial}{\partial \tau}, \quad \partial_{i}=\frac{\partial}{\partial z^{i}}, \\
& \hat{\gamma}^{\mu}=\left(\nabla_{a} \zeta^{\mu}\right) \gamma^{a}, \quad \hat{C}^{\mu \ldots \ldots}=\left(\nabla_{a} \zeta^{\mu}\right) \ldots C^{a \ldots}{ }_{b \ldots} \frac{\partial x^{b}}{\partial \zeta^{\nu}} \ldots, \\
& g_{\mu \nu}=\frac{\partial x^{a}}{\partial \zeta^{\mu}} \frac{\partial x^{b}}{\partial \zeta^{\nu}} \eta_{a b}, \quad g=\operatorname{det}\left(g_{\mu \nu}\right), \quad g_{(z)}=\operatorname{det}\left[\left(g_{i j}\right)_{i, j \leq 3}\right],
\end{aligned}
$$


Coordinates restricted to the indices $1,2,3$ will be written as $\hat{\gamma}^{i}, g_{i j}$ and $\hat{C}^{i \ldots}{ }_{j \ldots}$, and the zeroth coordinate will be indicated by $\tau$; thus, for instance: $\mathcal{A}_{a} \gamma^{a}=$ $\hat{\mathcal{A}}_{\mu} \hat{\gamma}^{\mu}=\hat{\mathcal{A}}_{\tau} \hat{\gamma}^{\tau}+\hat{\mathcal{A}}_{i} \hat{\gamma}^{i}$.

In these coordinates, the Dirac equation (2) and the product (3) take the form

$$
\begin{aligned}
& \left(\frac{i}{2}\left[|g|^{\frac{1}{2}} \hat{\gamma}^{\mu},|g|^{-\frac{1}{2}} \partial_{\mu}\right]_{+}-1\right) \psi=0, \\
& \left(\psi_{1}, \psi_{2}\right)_{\tau}=\int \overline{\psi_{1}} \hat{\gamma}^{\tau} \psi_{2}|g|^{\frac{1}{2}} d^{3} z .
\end{aligned}
$$

We now choose a Minkowski reference system $\left(e_{0}, \ldots, e_{3}\right)$. Let us denote

$$
n=\left[g^{\tau \tau}\right]^{-\frac{1}{2}} \nabla \tau
$$

and let $K$ be the Lorentz rotation of Dirac spinors in the hyperplane spanned by the pair of timelike unit vectors $\left(e_{0}, n\right)$, such that

$$
K^{-1} \gamma^{a} n_{a} K=\gamma^{0}, \quad K^{\dagger}=\gamma^{0} K^{-1} \gamma^{0},
$$

where dagger $\dagger$ denotes the Hermitian conjugation of matrices. The form and further properties of $K$ are discussed in Appendix A. We define the following transformation:

$$
\psi=T_{\tau} \chi, \quad T_{\tau}=\left(|g| g^{\tau \tau}\right)^{-\frac{1}{4}} K=\left|g_{(z)}\right|^{-\frac{1}{4}} K .
$$

The product (6) then takes the standard $\mathbb{C}^{4} \otimes L^{2}\left(\mathbb{R}^{3}\right)$ form

$$
\left(\psi_{1}, \psi_{2}\right)_{\tau}=\int \chi_{1}^{\dagger} \chi_{2} d^{3} z
$$

Setting $\psi$ into (5) and applying from the left the transformation $\left(g^{\tau \tau}\right)^{-\frac{1}{2}} T_{\tau}^{-1}$ we obtain an equivalent form of the Dirac equation

$$
\begin{aligned}
\left(\frac{i}{2}\right. & {\left.\left[\left(g^{\tau \tau}\right)^{-\frac{1}{2}} \hat{\gamma}_{K}^{\mu}, \partial_{\mu}\right]_{+}-\left(g^{\tau \tau}\right)^{-\frac{1}{2}}\right) \chi } \\
& +\frac{i}{2}\left(g^{\tau \tau}\right)^{-\frac{1}{2}}\left[\hat{\gamma}_{K}^{\mu},\left(K^{-1} \partial_{\mu} K\right)\right]+\chi=0,
\end{aligned}
$$

where we have introduced notation

$$
\gamma_{K}=K^{-1} \gamma K
$$

We now make an additional assumption: $\Sigma_{\tau}$ is rotationally symmetric in the chosen Minkowski system, that is $\tau=\tau\left(x^{0},|\mathbf{x}|\right)$. We show in Appendix A that in this case the anticommutator in the second line of (9) vanishes. We also note that $\left(g^{\tau \tau}\right)^{-\frac{1}{2}} \hat{\gamma}_{K}^{\tau}=\gamma^{0} \equiv \beta$. This allows us to write the Dirac equation in the form

$$
i \partial_{\tau} \chi=\left(-\frac{i}{2}\left[\left(g^{\tau \tau}\right)^{-\frac{1}{2}} \beta \hat{\gamma}_{K}^{i}, \partial_{i}\right]_{+}+\left(g^{\tau \tau}\right)^{-\frac{1}{2}} \beta\right) \chi .
$$

We summarize the above discussion.

Theorem 1. (i) Let the smooth function on the Minkowski space $\tau=\tau\left(x^{0},|\mathbf{x}|\right)$ determine its foliation by Cauchy surfaces $\Sigma_{\tau}$, with $\tau$ increasing into the future. Denote by $C_{0}^{\infty}\left(\Sigma_{\tau}, \mathbb{C}^{4}\right)$ the space of smooth, compactly supported functions on $\Sigma_{\tau}$ with values in $\mathbb{C}^{4}$. 
Then the free Dirac evolution of initial data in $C_{0}^{\infty}\left(\Sigma_{\sigma}, \mathbb{C}^{4}\right)$ is determined by a family of bijective linear evolution operators

$$
U_{0}^{\Sigma}(\tau, \sigma): C_{0}^{\infty}\left(\Sigma_{\sigma}, \mathbb{C}^{4}\right) \mapsto C_{0}^{\infty}\left(\Sigma_{\tau}, \mathbb{C}^{4}\right)
$$

isometric with respect to the products (3). The size of the support in $\Sigma_{\tau}$ is restricted by the size of the support in $\Sigma_{\sigma}$ and the relativistic causality. By continuity, $U_{0}^{\Sigma}(\tau, \sigma)$ extend to unitary operators

$$
U_{0}^{\Sigma}(\tau, \sigma): \mathcal{H}\left(\Sigma_{\sigma}\right) \mapsto \mathcal{H}\left(\Sigma_{\tau}\right),
$$

where $\mathcal{H}\left(\Sigma_{\tau}\right)$ is the Hilbert space of spinor functions on $\Sigma_{\tau}$ with the product (3).

(ii) Let $\left(\tau, z^{i}\right): M \mapsto \mathbb{R}^{4}$ be a smooth diffeomorphism, with notation (4), such that $\tau$ satisfies the assumptions of (i). Denote

$$
\begin{gathered}
\mathcal{H}=\mathbb{C}^{4} \otimes L^{2}\left(\mathbb{R}^{3}, d^{3} z\right), \\
H_{0}(\tau)=\frac{1}{2}\left[\lambda^{i}(\tau), p_{i}\right]_{+}+\mu(\tau), \quad h_{0}=\frac{1}{2}\left(\mathbf{p}^{2}+\mathbf{z}^{2}\right), \\
p_{i}=-i \partial / \partial z^{i}, \quad \mu(\tau)=\left(g^{\tau \tau}\right)^{-\frac{1}{2}} \beta, \quad \lambda^{i}(\tau)=\mu(\tau) \hat{\gamma}_{K}^{i} .
\end{gathered}
$$

Then the transformation

$$
T_{\tau}: \mathcal{H} \mapsto \mathcal{H}\left(\Sigma_{\tau}\right), \quad T_{\tau}=\left|g_{(z)}\right|^{-1 / 4} K,
$$

with operator $K$ discussed in Appendix A, is a unitary operator and the family

$$
U_{0}(\tau, \sigma)=T_{\tau}^{-1} U_{0}^{\Sigma}(\tau, \sigma) T_{\sigma}, \quad U_{0}(\tau, \sigma): \mathcal{H} \mapsto \mathcal{H}
$$

forms a unitary, strongly continuous evolution system, such that the following holds:

(A) $U_{0}(\tau, \sigma) C_{0}^{\infty}\left(\mathbb{R}^{3}, \mathbb{C}^{4}\right)=C_{0}^{\infty}\left(\mathbb{R}^{3}, \mathbb{C}^{4}\right)$ and the relativistic causality is respected.

(B) For $\varphi \in C_{0}^{\infty}\left(\mathbb{R}^{3}, \mathbb{C}^{4}\right)$ the maps $(\tau, \sigma) \mapsto H_{0}(\tau) U_{0}(\tau, \sigma) \varphi$ and $(\tau, \sigma) \mapsto$ $h_{0} U_{0}(\tau, \sigma) \varphi$ are strongly continuous, the map $(\tau, \sigma) \mapsto U_{0}(\tau, \sigma) \varphi$ is in the class $C^{1}$ in the strong sense, and the following equations are satisfied

$$
\begin{aligned}
& i \partial_{\tau} U_{0}(\tau, \sigma) \varphi=H_{0}(\tau) U_{0}(\tau, \sigma) \varphi, \\
& i \partial_{\sigma} U_{0}(\tau, \sigma) \varphi=-U_{0}(\tau, \sigma) H_{0}(\sigma) \varphi .
\end{aligned}
$$

(C) The operators $H_{0}(\tau)$ are symmetric on $C_{0}^{\infty}\left(\mathbb{R}^{3}, \mathbb{C}^{4}\right)$.

Proof. The symmetry of $H_{0}(\tau)$ follows from the symmetry of the operators $\lambda^{i}(\tau), \mu(\tau)$ and $p_{i}$, and invariance of $C_{0}^{\infty}\left(\mathbb{R}^{3}, \mathbb{C}^{4}\right)$ with respect to them. The other statements of the theorem follow easily from the discussion preceding the theorem.

We now add assumptions which will be satisfied in the context of our application, and which allow us to significantly extend the domains of validity of the results in the last theorem. We use the abbreviation

$$
\langle z\rangle=\left(|\mathbf{z}|^{2}+1\right)^{\frac{1}{2}} .
$$

Also, the usual multi-index notation will be used. 
Theorem 2. Let all the assumptions of Theorem 1 be satisfied. Suppose in addition that the following bounds of matrix norms hold:

$$
\begin{aligned}
& \left|\lambda^{i}(\tau, \mathbf{z})\right| \leq C(\tau)\langle z\rangle, \quad\left|\partial_{z}^{\alpha} \lambda^{i}(\tau, \mathbf{z})\right| \leq C(\tau), \quad 1 \leq|\alpha| \leq 3 \\
& \left|\partial_{z}^{\beta} \mu(\tau, \mathbf{z})\right| \leq C(\tau)\langle z\rangle^{2-|\beta|}, \quad|\beta| \leq 2,
\end{aligned}
$$

where $C(\tau)$ is a continuous function. Denote $h_{0}=\frac{1}{2}\left(\mathbf{p}^{2}+\mathbf{z}^{2}\right)$, the self-adjoint harmonic oscillator Hamiltonian.

Then the following holds:

(A) $H_{0}(\tau)$ are essentially self-adjoint on $C_{0}^{\infty}\left(\mathbb{R}^{3}, \mathbb{C}^{4}\right)$ and on each core of $h_{0}$, and $\mathcal{D}\left(h_{0}\right) \subseteq \mathcal{D}\left(H_{0}(\tau)\right)$. Moreover, $h_{0}^{-1} H_{0}(\tau)$ and $H(\tau) h_{0}^{-1}$ extend to bounded operators, and as functions of $\tau$ are strongly continuous.

(B) The operator $h_{0} U_{0}(\tau, \sigma) h_{0}^{-1}$ is bounded, with the norm

$$
\left\|h_{0} U_{0}(\tau, \sigma) h_{0}^{-1}\right\| \leq \exp \left[\text { const } \int_{\sigma}^{\tau} C(\rho) d \rho\right],
$$

so in particular

$$
U_{0}(\tau, \sigma) \mathcal{D}\left(h_{0}\right)=\mathcal{D}\left(h_{0}\right),
$$

and the map $(\tau, \sigma) \mapsto h_{0} U(\tau, \sigma) h_{0}^{-1}$ is strongly continuous.

(C) For $\varphi \in \mathcal{D}\left(h_{0}\right)$ the vector functions $h_{0} U_{0}(\tau, \sigma) \varphi, H_{0}(\tau) U_{0}(\tau, \sigma) \varphi$ and $U_{0}(\tau, \sigma) H_{0}(\sigma) \varphi$ are strongly continuous with respect to parameters, the vector function $U_{0}(\tau, \sigma) \varphi$ is of the class $C^{1}$ with respect to parameters in the strong sense and the following equations hold

$$
\begin{aligned}
& i \partial_{\tau} U_{0}(\tau, \sigma) \varphi=H_{0}(\tau) U_{0}(\tau, \sigma) \varphi, \\
& i \partial_{\sigma} U_{0}(\tau, \sigma) \varphi=-U_{0}(\tau, \sigma) H_{0}(\sigma) \varphi .
\end{aligned}
$$

(D) Relativistic causality is respected by $U_{0}(\tau, \sigma)$. Therefore, if we denote

$\mathcal{H} \supset \mathcal{H}_{c}$-the subspace of functions with compact essential support, $\mathcal{D}_{c}\left(\mathbf{p}^{2}\right)=\mathcal{H}_{c} \cap \mathcal{D}\left(\mathbf{p}^{2}\right)$,

then

$$
U_{0}(\tau, \sigma) \mathcal{H}_{c}=\mathcal{H}_{c}, \quad U_{0}(\tau, \sigma) \mathcal{D}_{c}\left(\mathbf{p}^{2}\right)=\mathcal{D}_{c}\left(\mathbf{p}^{2}\right)
$$

Proof. It follows from the assumptions on $\lambda^{i}$ and $\mu$ that $H_{0}(\tau)$ fulfill all the conditions imposed on the operator $h$ in Lemma 14 in Appendix C. In Theorems 12 and 13 in Appendix B, we now put

$$
\mathcal{D}=C_{0}^{\infty}\left(\mathbb{R}^{3}, \mathbb{C}^{4}\right), \quad h(\tau)=H_{0}(\tau), \quad u(\tau, \sigma)=U_{0}(\tau, \sigma)
$$

and $h_{0}$ as defined in the present assumptions. Then, by the results of Theorem 1, all the assumptions of these theorems are satisfied and the present thesis follows. 


\section{Evolution in External Field}

We now consider the Dirac equation in an external field, of the form

$$
\left(\frac{1}{2}\left[\gamma^{a}, i \nabla_{a}\right]_{+}-1-V\right) \psi=0,
$$

where $V(x)$ is a matrix function satisfying the condition

$$
V(x)^{\dagger}=\gamma^{0} V(x) \gamma^{0}
$$

which guarantees the reality of the interaction term $-\bar{\psi} V \psi$ in the Lagrangian and the conservation of the current $\bar{\psi} \gamma^{a} \psi$. The addition of this interaction term leads to the modification of the free curvilinear version to the equation of the form

$$
i \partial_{\tau} \varphi=\left(\frac{1}{2}\left[\lambda^{i}, p_{i}\right]_{+}+\mu+W\right) \varphi
$$

where

$$
W(\tau, \mathbf{z})=\mu K^{-1} V(x) K=W(\tau, \mathbf{z})^{\dagger},
$$

Hermicity being equivalent to the property (14) [see (8)].

Using the interaction picture technique, we shall obtain the evolution operators for which Eq. (15) is satisfied. However, thanks to the relativistic causality we can extend the applicability of the technique to the following setting, in which the operators $W(\tau)$ do not need to be bounded.

Theorem 3. Let all the assumptions of Theorems 1 and 2 be satisfied. Suppose in addition that $W(\tau, \mathbf{z})$ is a Hermitian matrix function such that the mappings

$$
\left\{\mathbb{R} \ni \tau \mapsto \partial_{z}^{\alpha} W(\tau, \mathbf{z})\right\} \in C^{0}\left(\mathbb{R}, L_{\mathrm{loc}}^{\infty}\left(\mathbb{R}^{3}\right)\right), \quad|\alpha| \leq 2,
$$

and

$$
\left\|\langle z\rangle^{-2+|\alpha|} \partial_{z}^{\alpha} W(\tau, \mathbf{z})\right\|_{\infty} \leq C(\tau), \quad|\alpha| \leq 1,
$$

where $C(\tau)$ is a continuous function. Denote

$$
H(\tau)=H_{0}(\tau)+W(\tau),
$$

with the initial domain $C_{0}^{\infty}\left(\mathbb{R}^{3}, \mathbb{C}^{4}\right)$, and define the formal series

$$
\begin{aligned}
& U(\tau, \sigma)=\sum_{n=0}^{\infty} U^{(n)}(\tau, \sigma), \quad U^{(0)}(\tau, \sigma)=\mathbf{1}, \\
& U^{(n)}(\tau, \sigma)=(-i)^{n} \int_{\tau \geq \tau_{n} \geq \tau_{1} \geq \sigma} U_{0}\left(\tau, \tau_{n}\right) W\left(\tau_{n}\right) U_{0}\left(\tau_{n}, \tau_{n-1}\right) \ldots \\
& \tau \geq \tau_{n} \geq \ldots \geq \tau_{1} \geq \sigma \\
& \ldots W\left(\tau_{1}\right) U_{0}\left(\tau_{1}, \sigma\right) \mathrm{d} \tau_{n} \ldots \mathrm{d} \tau_{1} \\
& =-i \int_{\sigma}^{\tau} U_{0}(\tau, \rho) W(\rho) U^{(n-1)}(\rho, \sigma) \mathrm{d} \rho \\
& =-i \int_{\sigma}^{\tau} U^{(n-1)}(\tau, \rho) W(\rho) U_{0}(\rho, \sigma) \mathrm{d} \rho, \quad n \geq 1 .
\end{aligned}
$$

Then the following is true: 
(A) $H(\tau)$ are essentially self-adjoint on $C_{0}^{\infty}\left(\mathbb{R}^{3}, \mathbb{C}^{4}\right)$ and on each core of $h_{0}$, and $\mathcal{D}\left(h_{0}\right) \subseteq \mathcal{D}(H(\tau))$.

(B) Series $U(\tau, \sigma)$ and its conjugation converge strongly on $\mathcal{H}_{c}$, and the limit operator extends to a unitary propagator on $\mathcal{H}$, strongly continuous in its parameters.

(C) $U(\tau, \sigma)$ respects relativistic causality and

$$
U(\tau, \sigma) \mathcal{D}_{c}\left(\mathbf{p}^{2}\right)=\mathcal{D}_{c}\left(\mathbf{p}^{2}\right) .
$$

(D) For $\varphi \in \mathcal{D}_{c}\left(\mathbf{p}^{2}\right)$ the vector functions $h_{0} U(\tau, \sigma) \varphi, H(\tau) U(\tau, \sigma) \varphi$ and $U(\tau, \sigma) H(\sigma) \varphi$ are strongly continuous with respect to parameters, the function $U(\tau, \sigma) \varphi$ is of the class $C^{1}$ with respect to parameters in the strong sense and the following equations hold

$$
\begin{aligned}
& i \partial_{\tau} U(\tau, \sigma) \varphi=H(\tau) U(\tau, \sigma) \varphi, \\
& i \partial_{\sigma} U(\tau, \sigma) \varphi=-U(\tau, \sigma) H(\sigma) \varphi .
\end{aligned}
$$

(E) Unitary operators satisfying $(D)$, with $U(\sigma, \sigma)=\mathbf{1}$, are unique.

Proof. The proof of (A) is similar as the proof in the free case, statement (A) in Theorem 2. One has to note that the assumption (17) ensures the validity of items (i) and (ii) in Lemma 14; the result of item (iii) is not needed.

Consider the series (18). As each of the operators $U_{0}\left(\tau, \tau_{n}\right), U_{0}\left(\tau_{k}, \tau_{k-1}\right)$ and $U_{0}\left(\tau_{1}, \sigma\right)$ in the definition of $U^{(n)}(\tau, \sigma)$ respects causality, and multiplication by $W\left(\tau_{k}, \mathbf{z}\right)$ does not enlarge the support of the function, each of the operators $U^{(n)}(\tau, \sigma)$ respects causality. Let, for chosen $T$ and $r$,

$$
\tau, \sigma \in[-T, T], \quad \varphi \in \mathcal{H}, \quad \operatorname{ess} \operatorname{supp} \varphi \subseteq\{\mathbf{z}|| \mathbf{z} \mid \leq r\},
$$

which is assumed for the rest of this proof. Then there exists $R(T, r)$ such that the essential support of all functions $U^{(n)}(\tau, \sigma) \varphi$ is contained in $|\mathbf{z}| \leq R(T, r)$ (uniformly with respect to $\tau, \sigma$ and $\varphi$ in the assumed range). Let $J(\mathbf{z})$ be a smooth function such that $J(\mathbf{z})=1$ for $|\mathbf{z}| \leq R$ and $J(\mathbf{z})=0$ for $|\mathbf{z}| \geq R+1$. Denote by $U_{J}(\tau, \sigma)$ and $U_{J}^{(n)}(\tau, \sigma)$ the series (18) and its terms in which $W(\rho, \mathbf{z})$ have been replaced by $W_{J}(\rho, \mathbf{z})=J(\mathbf{z}) W(\rho, \mathbf{z})$; note that by assumption (16) $W_{J}(\rho)$ is a bounded, strongly continuous operator, with $\left\|W_{J}(\rho)\right\| \leq d_{1}$ for some constant $d_{1}$, uniformly on $\rho \in[-T, T]$. Using the first of the recursive relations in (18), it is now easy to see that in the given setting

$$
U^{(n)}(\tau, \sigma) \varphi=U_{J}^{(n)}(\tau, \sigma) \varphi .
$$

Arguing as in the proof of the Dyson expansion (see Thm. X.69 in [15]), one shows that $U_{J}(\tau, \sigma)$ converges uniformly to a strongly continuous unitary propagator. As the space $\mathcal{H}_{c}$ is dense in $\mathcal{H}$, the statement (B) is proved. Also, the causality is respected.

To prove (C) (the invariance of the subspace) and (D), we write

$$
h_{0} W_{J}(\rho) h_{0}^{-1}=\left[h_{0}, W_{J}(\rho)\right] h_{0}^{-1}+W_{J}(\rho)
$$

and note that by Theorem 12 applied to $h(\tau)=W_{J}(\tau)$ (with Lemma 14) the first term on the rhs is a bounded operator, strongly continuous by the assumption (16). Therefore, the operator $h_{0} W_{J}(\rho) h_{0}^{-1}$ is also bounded and 
strongly continuous, and $\left\|h_{0} W_{J}(\rho) h_{0}^{-1}\right\| \leq d_{2}$ for some constant $d_{2}$, uniformly on $\rho \in[-T, T]$. Taking also into account statement (B) in Theorem 2, we conclude that $h_{0} U_{J}^{(n)}(\tau, \sigma) h_{0}^{-1}$ is bounded, strongly continuous, with the norm estimated by

$$
\left\|h_{0} U_{J}^{(n)}(\tau, \sigma) h_{0}^{-1}\right\| \leq(n !)^{-1}\left(d_{1}+d_{2}\right)^{n} \exp \left[\operatorname{const} \int_{\sigma}^{\tau} C(\rho) \mathrm{d} \rho\right],
$$

This implies that the series $\sum_{n} h_{0} U_{J}^{(n)}(\tau, \sigma) h_{0}^{-1}$ converges uniformly to the strongly continuous function $h_{0} U_{J}(\tau, \sigma) h_{0}^{-1}$. Let us now impose on $\varphi$ a stronger assumption that $\varphi \in \mathcal{D}_{c}\left(\mathbf{p}^{2}\right)$. Then

$$
h_{0} U(\tau, \sigma) \varphi=h_{0} U_{J}(\tau, \sigma) \varphi=h_{0} U_{J}(\tau, \sigma) h_{0}^{-1} h_{0} \varphi .
$$

This proves $(\mathrm{C})$, and also the strong continuity of $h_{0} U(\tau, \sigma) \varphi$ in (D). The strong continuity of $H(\tau) U(\tau, \sigma) \varphi$ and $U(\tau, \sigma) H(\sigma) \varphi$ follows now from the strong continuity of $H(\tau) h_{0}^{-1}$, similarly as in Theorem 2, basing on Theorem 12 and assumptions (16) and (17).

Next, we note that

$$
U_{0}(0, \tau) U^{(n)}(\tau, \sigma) \varphi=-i \int_{\sigma}^{\tau} U_{0}(0, \rho) W(\rho) U^{(n-1)}(\rho, \sigma) \varphi \mathrm{d} \rho,
$$

so this vector function is continuously differentiable in $\tau$ in the strong sense and

$$
i \partial_{\tau}\left[U_{0}(0, \tau) U^{(n)}(\tau, \sigma)\right] \varphi=U_{0}(0, \tau) W(\tau) U^{(n-1)}(\tau, \sigma) \varphi
$$

(remember that on the rhs $W$ may be replaced by $W_{J}$ ). Again, using the uniform convergence of $U_{J}(\tau, \sigma)$ we obtain

$$
i \partial_{\tau}\left[U_{0}(0, \tau) U(\tau, \sigma)\right] \varphi=U_{0}(0, \tau) W(\tau) U(\tau, \sigma) \varphi,
$$

and the rhs is strongly continuous in $(\tau, \sigma)$. Finally, differentiating

$$
U(\tau, \sigma) \varphi=U_{0}(\tau, 0)\left[U_{0}(0, \tau) U(\tau, \sigma)\right] \varphi
$$

by the Leibnitz rule and using $(\mathrm{C})$ from Theorem 2 we arrive at the first equation in (19). The uniqueness (E) follows easily from this equation. The proof of the second equation in (19) is similar, with the use of the second of the recursive relations in (18); we omit the details.

The most general matrix field $V(x)$ satisfying condition (14) may be concisely represented in the form

$$
V=\sum_{k=0}^{4} i^{\frac{1}{2} k(k-1)} C_{a_{1} \ldots a_{k}}^{k} \gamma^{a_{1}} \ldots \gamma^{a_{k}},
$$

where the tensor fields $C_{a_{1} \ldots a_{k}}^{k}$ are real and antisymmetric. The corresponding form of the Hermitian matrix function $W$ is

$$
W=\mu \sum_{k=0}^{4} i^{\frac{1}{2} k(k-1)} \hat{C}_{\mu_{1} \ldots \mu_{k}}^{k} \hat{\gamma}_{K}^{\mu_{1}} \cdots \hat{\gamma}_{K}^{\mu_{k}} .
$$

This form encompasses the scalar $(k=0)$ and pseudoscalar $(k=4)$ potentials, the electromagnetic vector potential $(k=1)$ and the pseudovector potential 
$(k=3)$, the interactions characteristic for anomalous magnetic and electric moments, as well as the linearized gravitation $(k=2)$.

\section{Special Foliation, New Picture and Free Asymptotics}

We now choose the foliation $\tau$ and the variables $\mathbf{z}$ by

$$
x^{0}=\tau\langle z\rangle \equiv \tau\left(|\mathbf{z}|^{2}+1\right)^{\frac{1}{2}}, \quad \mathbf{x}=\langle\tau\rangle \mathbf{z} \equiv\left(\tau^{2}+1\right)^{\frac{1}{2}} \mathbf{z},
$$

and we also denote

$$
\langle\tau \mid z\rangle=\left(\tau^{2}+|\mathbf{z}|^{2}+1\right)^{\frac{1}{2}} .
$$

The idea behind this choice is that for $\tau$ tending to $\pm \infty$ the Cauchy surfaces should tend, for timelike directions in the spacetime, to the hyperboloids $x^{2}=\tau^{2}$. All geometrical facts on these curvilinear coordinates needed for our purposes are gathered in Appendix D.

From now on, we adopt the coordinate system (20). Using the properties of this system, it is then easy to see that all the conditions of Theorems 1 and 2 are satisfied. Also, a convenient property of these coordinates is that $g_{\tau i}=0$. Therefore, we find

$$
\left[\hat{\gamma}_{K}^{i}, \beta\right]_{+}=\left(g^{\tau \tau}\right)^{-\frac{1}{2}} K^{-1}\left[\hat{\gamma}^{i}, \hat{\gamma}^{\tau}\right]_{+} K=2\left(g^{\tau \tau}\right)^{-\frac{1}{2}} g^{i \tau}=0,
$$

and then

$$
\begin{aligned}
& {\left[\lambda^{i}, \beta\right]_{+}=0=\left[\lambda^{i}, \mu\right]_{+},} \\
& \frac{1}{2}\left[\lambda^{i}, \lambda^{j}\right]_{+}=-g_{\tau \tau} g^{i j} \mathbf{1} \equiv \rho^{i j} \mathbf{1} ;
\end{aligned}
$$

the symmetric form $\rho$ is positive definite.

The free evolution $U_{0}(\tau, \sigma)$ may be easily expressed in the coordinate system (20). For this purpose, it is sufficient to consider $U_{0}(\tau, 0)$. Using the representation of the Dirac solution (103) in Appendix E and the definition (12), for $\varphi \in \mathcal{S}\left(\mathbb{R}^{3}, \mathbb{C}^{4}\right)$ we obtain

$$
\begin{aligned}
{\left[U_{0}(\tau, 0) \varphi\right](\mathbf{z})=} & \left(\frac{\langle\tau \mid z\rangle}{\langle\tau\rangle\langle z\rangle}\right)^{\frac{1}{2}} K(\tau, \mathbf{z})^{-1} \\
& \times\left(\frac{\langle\tau\rangle}{2 \pi}\right)^{\frac{3}{2}} \int\left[e^{-i x(\tau, \mathbf{z}) \cdot v} P_{+}(v)-e^{i x(\tau, \mathbf{z}) \cdot v} P_{-}(v)\right]\left[\mathcal{F}^{-1} \varphi\right](v) \mathrm{d} \mu(v),
\end{aligned}
$$

where $x(\tau, \mathbf{z})=(\tau\langle z\rangle,\langle\tau\rangle \mathbf{z})$. We consider the asymptotic form of this evolution for $\tau \rightarrow \pm \infty$. Let $\varphi \in \mathcal{F} C_{0}^{\infty}\left(\mathbb{R}^{3}, \mathbb{C}^{4}\right)$ and restrict $\mathbf{z}$ to a compact set. Then both $\mathbf{v}$ (the space part of $v$ ) and $\mathbf{z}$ are restricted to bounded sets and by the stationary phase method (see, e.g., [19]) the leading asymptotic behavior of the second line in (23) is given by

$$
\mp i\left[e^{-i\left(\tau \pm \frac{\pi}{4}\right)} P_{+}\left(z^{0}, \pm \mathbf{z}\right)+e^{i\left(\tau \pm \frac{\pi}{4}\right)} P_{-}\left(z^{0}, \pm \mathbf{z}\right)\right]\left[\mathcal{F}^{-1} \varphi\right]\left(z^{0}, \pm \mathbf{z}\right)+O\left(\langle\tau\rangle^{-1}\right),
$$

where $z^{0}=\langle z\rangle$, the upper/lower signs $\mp$ and \pm correspond to the limits $\tau \rightarrow \pm \infty$, respectively, and the rest is bounded uniformly for $\mathbf{z}$ in the given 
set. The limit of the term on the rhs of (23) in the first line is equal to $\langle z\rangle^{-\frac{1}{2}} K( \pm \infty, \mathbf{z})^{-1}$, with

$$
K( \pm \infty, \mathbf{z})=2^{-\frac{1}{2}}\left[(1+\langle z\rangle)^{\frac{1}{2}} \pm(1+\langle z\rangle)^{-\frac{1}{2}} \gamma^{0} \mathbf{z} \cdot \gamma\right]=K(\infty, \pm \mathbf{z})
$$

and again the rest is bounded by $\operatorname{const}\langle\tau\rangle^{-1}$, uniformly in the given set. We now note two facts:

$$
\begin{aligned}
& K(\infty, \mathbf{v})^{-1} v \cdot \gamma K(\infty, \mathbf{v})=\beta, \\
& e^{-i \sigma} \frac{1}{2}(\mathbf{1}+\beta)+e^{i \sigma} \frac{1}{2}(\mathbf{1}-\beta)=e^{-i \sigma \beta} .
\end{aligned}
$$

The first identity is the limit form of the first of relations in (8), but it may also be checked directly. The second identity is most easily evaluated on the two complementary eigenspaces of $\beta$ (with eigenvalues \pm 1 ).

Setting the asymptotic forms (24) and (25) into formula (23), and using the above identities (the second one with $\sigma=\tau \pm \frac{\pi}{4}$ ), we obtain

$$
\begin{aligned}
{\left[U_{0}(\tau, 0) \varphi\right](\mathbf{z})=} & \mp i e^{-i\left(\tau \pm \frac{\pi}{4}\right) \beta}\langle z\rangle^{-\frac{1}{2}} K(\infty, \pm \mathbf{z})^{-1}\left[\mathcal{F}^{-1} \varphi\right]\left(z^{0}, \pm \mathbf{z}\right) \\
& +O\left(\langle\tau\rangle^{-1}\right) .
\end{aligned}
$$

The last formula suggests the definition of the following unitary transformation:

$$
\Phi(\tau)=\exp [-i \tau \beta]
$$

and the associated change of the evolution 'picture' (also in the interacting case):

$$
\begin{aligned}
& U_{0 \Phi}(\tau, \sigma)=\Phi(\tau)^{*} U_{0}(\tau, \sigma) \Phi(\sigma), \quad U_{\Phi}(\tau, \sigma)=\Phi(\tau)^{*} U(\tau, \sigma) \Phi(\sigma), \\
& H_{0 \Phi}(\tau)=\Phi(\tau)^{*} H_{0}(\tau) \Phi(\tau)-\beta, \\
& H_{\Phi}(\tau)=H_{0 \phi}(\tau)+W_{\Phi}(\tau), \quad W_{\Phi}(\tau)=\Phi(\tau)^{*} W(\tau) \Phi(\tau) .
\end{aligned}
$$

Remark 4. Under the conditions of Theorem 3, all statements of its thesis remain valid with the replacements defined by Eqs. (27).

This follows quite trivially, as $\Phi(\tau)$ acts only on the factor $\mathbb{C}^{4}$ in the Hilbert space $\mathcal{H}=\mathbb{C}^{4} \otimes L^{2}\left(\mathbb{R}^{3}\right)$.

After that, we go back to the asymptotics of the free evolution, where we shall need the parity operator

$$
[\mathcal{P} \varphi](\mathbf{z})=\varphi(-\mathbf{z}) .
$$

Moreover, we observe that the map

$$
[\mathcal{K} \varphi](\mathbf{z})=\langle z\rangle^{\frac{1}{2}} K(\infty, \mathbf{z}) \varphi(\mathbf{z})
$$

is a unitary operator $\mathcal{H} \mapsto L_{\gamma}^{2}(H)$ (with the latter space defined in Appendix E).

Theorem 5. The following strong limits exist as unitary operators in $\mathcal{H}$ :

$$
\begin{aligned}
& U_{0 \Phi}(+\infty, 0)=\underset{\tau \rightarrow+\infty}{\mathrm{s}-\lim _{0 \Phi}} U_{0 \Phi}(\tau, 0)=-i e^{-i \frac{\pi}{4} \beta} \mathcal{K}^{-1} \mathcal{F}^{-1}, \\
& U_{0 \Phi}(-\infty, 0)=\underset{\tau \rightarrow-\infty}{\mathrm{s}-\lim _{0 \Phi}} U_{0 \Phi}(\tau, 0)=i e^{+i \frac{\pi}{4} \beta} \mathcal{P} \mathcal{K}^{-1} \mathcal{F}^{-1},
\end{aligned}
$$




$$
\begin{aligned}
& U_{0 \Phi}(0,+\infty)=\underset{\tau \rightarrow+\infty}{\mathrm{s}-\lim _{\tau}} U_{0 \Phi}(0, \tau)=i \mathcal{F} \mathcal{K} e^{+i \frac{\pi}{4} \beta}, \\
& U_{0 \Phi}(0,-\infty)=\underset{\tau \rightarrow-\infty}{\mathrm{s}-\lim } U_{0 \Phi}(0, \tau)=-i \mathcal{F} \mathcal{K} \mathcal{P} e^{-i \frac{\pi}{4} \beta}, \\
& U_{0 \Phi}(0, \pm \infty)=U_{0 \Phi}( \pm \infty, 0)^{*}
\end{aligned}
$$

Therefore, ${ }^{8}$

$$
U_{0 \Phi}(+\infty,-\infty)=i \beta \mathcal{P} .
$$

Proof. For $\psi \in C_{0}^{\infty}\left(\mathbb{R}^{3}, \mathbb{C}^{4}\right)$ and $\varphi \in \mathcal{F} C_{0}^{\infty}\left(\mathbb{R}^{3}, \mathbb{C}^{4}\right)$ formula (26) gives

$$
\begin{aligned}
\lim _{\tau \rightarrow+\infty}\left(\psi, U_{0 \Phi}(\tau, 0) \varphi\right) & =\left(\psi,(-i) e^{-i \frac{\pi}{4} \beta} \mathcal{K}^{-1} \mathcal{F}^{-1} \varphi\right), \\
\lim _{\tau \rightarrow-\infty}\left(\psi, U_{0 \Phi}(\tau, 0) \varphi\right) & =\left(\psi, i e^{+i \frac{\pi}{4} \beta} \mathcal{P} \mathcal{K}^{-1} \mathcal{F}^{-1} \varphi\right) .
\end{aligned}
$$

Both subspaces are dense in $\mathcal{H}$, so the weak operator limits result. But the limit operators are evidently unitary, so the weak limits imply the strong limits of $U_{0 \Phi}(\tau, 0)$, as well as its conjugate.

\section{Electromagnetic Interaction and Gauge Transformation}

In the rest of this article we are interested in the standard, minimal coupling electromagnetic interaction. For the electromagnetic field $F_{a b}$, we reserve notation $A_{a}$ for the Lorenz gauge potential (fully specified in what follows). We write $\mathcal{A}_{a}$ for the potential in a general gauge to be used in the Dirac equation. Also, we recall our conventions defined in formulas (4) and the following remarks, so $\hat{F}_{\mu \nu}, \hat{A}_{\mu}$ and $\hat{\mathcal{A}}_{\mu}$ are components of these fields in our coordinate system, and for $\mu, \nu=i, j$, etc. the range is restricted to values $1,2,3$.

Therefore, in the electromagnetic case the field $V$ and its transformed version $W$ are

$$
V(x)=\mathcal{A}_{a}(x) \gamma^{a}, \quad W(\tau, \mathbf{z})=\hat{\mathcal{A}}_{\tau}(\tau, \mathbf{z})+\hat{\mathcal{A}}_{i}(\tau, \mathbf{z}) \lambda^{i}(\tau, \mathbf{z}),
$$

and we write the Hamiltonian as

$$
H=\frac{1}{2}\left[\lambda^{i}, \pi_{i}\right]_{+}+\mu+\hat{\mathcal{A}}_{\tau}, \quad \pi_{i}=p_{i}+\hat{\mathcal{A}}_{i} .
$$

Theorem 6. The coordinate system $(\tau, \mathbf{z})$ given by $(20)$ is assumed.

(i) Let the electromagnetic potential $\mathcal{A}(x)$ have components $\hat{\mathcal{A}}_{\mu}(\tau, \mathbf{z})$ such that for all indices $\mu=\tau, 1,2,3$ the mappings

$$
\left\{\mathbb{R} \ni \tau \mapsto \partial_{z}^{\alpha} \hat{\mathcal{A}}_{\mu}(\tau, \mathbf{z})\right\} \in C^{0}\left(\mathbb{R}, L_{\mathrm{loc}}^{\infty}\left(\mathbb{R}^{3}\right)\right), \quad|\alpha| \leq 2,
$$

and

$$
\left.\begin{array}{l}
\left\|\langle z\rangle^{-2+|\alpha|} \partial_{z}^{\alpha} \hat{\mathcal{A}}_{\tau}(\tau, \mathbf{z})\right\|_{\infty} \\
\left\|\langle z\rangle^{-1+|\alpha|} \partial_{z}^{\alpha} \hat{\mathcal{A}}_{i}(\tau, \mathbf{z})\right\|_{\infty}
\end{array}\right\} \leq C(\tau), \quad|\alpha| \leq 1,
$$

where $C(\tau)$ is a continuous function. Then the conditions of Theorem 3 are fulfilled and the unitary propagator $U(\tau, \sigma)$ with the listed properties is obtained.

\footnotetext{
${ }^{8}$ Note an analogy with the nonrelativistic case, see Section 2 in [11].
} 
(ii) Let the potential $\mathcal{A}(x)$ and the corresponding propagator $U(\tau, \sigma)$ be as in (i). Define a new gauge

$$
\mathcal{A}^{\mathcal{G}}=\mathcal{A}-\nabla \mathcal{G}
$$

where $\mathcal{G}(x)$ is a gauge function such that for all indices $\mu=\tau, 1,2,3$ the mappings

$$
\left\{\mathbb{R} \ni \tau \mapsto \partial_{z}^{\alpha} \partial_{\mu} \mathcal{G}(\tau, \mathbf{z})\right\} \in C^{0}\left(\mathbb{R}, L_{\mathrm{loc}}^{\infty}\left(\mathbb{R}^{3}\right)\right), \quad|\alpha| \leq 1
$$

and

$$
\left.\begin{array}{l}
\left\|\langle z\rangle^{-2+|\alpha|} \partial_{z}^{\alpha} \partial_{\tau} \mathcal{G}(\tau, \mathbf{z})\right\|_{\infty} \\
\left\|\langle z\rangle^{-1+|\alpha|} \partial_{z}^{\alpha} \partial_{i} \mathcal{G}(\tau, \mathbf{z})\right\|_{\infty}
\end{array}\right\} \leq C(\tau), \quad|\alpha| \leq 1,
$$

where $C(\tau)$ is a continuous function. Denote by $H^{\mathcal{G}}(\tau)$ the interacting Hamiltonian with $\hat{\mathcal{A}}$ replaced by $\hat{\mathcal{A}}^{\mathcal{G}}$, and

$$
U^{\mathcal{G}}(\tau, \sigma)=e^{i \mathcal{G}(\tau)} U(\tau, \sigma) e^{-i \mathcal{G}(\sigma)} .
$$

Then for such modified operators the statements $(A)$ and $(C)-(E)$ of Theorem 3 are satisfied.

Proof. (i) The bounds (13) are satisfied in our coordinate system. Together with the assumed properties of $\hat{\mathcal{A}}_{\mu}$, this ensures that the interaction term (28) satisfies the assumptions of Theorem 3, so the thesis follows.

(ii) For (A) we note that the assumption (32) implies that the interaction term $W^{\mathcal{G}}=\hat{\mathcal{A}}_{\tau}^{\mathcal{G}}+\hat{\mathcal{A}}_{i}^{\mathcal{G}} \lambda^{i}$ satisfies assumptions imposed on $M$ in Lemma 14 (i) and (ii) (not necessarily (iii)). This is sufficient for the conclusion on self-adjointness of $H^{\mathcal{G}}$ obtained as in Theorem 3. Moreover, it follows from (31) that $e^{i \mathcal{G}(\tau)} \mathcal{D}_{c}\left(\mathbf{p}^{2}\right)=\mathcal{D}_{c}\left(\mathbf{p}^{2}\right)$, so (C) is satisfied. Finally, for $\varphi \in \mathcal{D}_{c}\left(\mathbf{p}^{2}\right)$ we have

$$
\begin{aligned}
& i \partial_{\tau} e^{i \mathcal{G}(\tau)} \varphi=-e^{i \mathcal{G}(\tau)} \partial_{\tau} \mathcal{G}(\tau) \varphi, \\
& e^{i \mathcal{G}(\tau)} H(\tau) e^{-i \mathcal{G}(\tau)} \varphi=\left[H(\tau)-\lambda^{i}(\tau) \partial_{i} \mathcal{G}(\tau)\right] \varphi,
\end{aligned}
$$

so the remaining statements easily follow with the use of (31).

\section{Scattering}

We come here to our main objective in this article, scattering of the Dirac field in an external time-dependent electromagnetic field. With the assumptions of Theorem 6, augmented by some decay conditions formulated in the two assumptions below, we shall obtain the complete description of scattering in terms of the Cauchy surfaces of constant $\tau$, as to be found in Theorem 7 . The existence of the wave operators needs only a rather simple additional Assumption I, but their unitarity and completeness are more demanding, and they follow from Assumption II. It is with regard to this latter question that we need to discuss some further notation and properties.

It is easy to see that the operators

$$
\tilde{H}=\frac{1}{2}\left[\lambda^{i}, \pi_{i}\right]_{+}=H-\mu-\hat{\mathcal{A}}_{\tau}, \quad \tilde{H}_{0}=\frac{1}{2}\left[\lambda^{i}, p_{i}\right]_{+}=H_{0}-\mu
$$


have similar properties as $H$ and $H_{0}$, in particular they are essentially selfadjoint on $C_{0}^{\infty}\left(\mathbb{R}^{3}, \mathbb{C}^{4}\right)$, so $\mathcal{D}_{c}(\mathbf{p})=\mathcal{H}_{c} \cap \mathcal{D}(\mathbf{p})$ is contained in their domains. Therefore, $\mathcal{D}_{c}\left(\mathbf{p}^{2}\right)$ is contained in the domains of $\tilde{H}^{2}$ and $\tilde{H}_{0}^{2}$ [as well as $H^{2}$ and $H_{0}^{2}$. Moreover, we note for later use that

$$
[\tilde{H}, \beta]_{+}=\left[\tilde{H}_{0}, \beta\right]_{+}=0,
$$

which is a consequence of (21).

We shall need a more explicit form of $\tilde{H}^{2}$ below. We calculate (with $\rho$ defined in (22))

$$
\begin{aligned}
\tilde{H}^{2} & =\left(\pi_{i} \lambda^{i}+\frac{i}{2} \partial \cdot \lambda\right)\left(\lambda^{j} \pi_{j}-\frac{i}{2} \partial \cdot \lambda\right) \\
& =\pi_{i} \rho^{i j} \pi_{j}+\frac{1}{2} \pi_{i}\left[\lambda^{i}, \lambda^{j}\right] \pi_{j}+\frac{i}{2}(\partial \cdot \lambda) \lambda^{j} \pi_{j}-\frac{i}{2} \pi_{j} \lambda^{j}(\partial \cdot \lambda)+\frac{1}{4}(\partial \cdot \lambda)^{2} .
\end{aligned}
$$

The second term on the rhs above may be written in two alternative ways:

$$
\begin{aligned}
\frac{1}{2} \pi_{i}\left[\lambda^{i}, \lambda^{j}\right] \pi_{j} & =-\frac{i}{2}\left(\partial_{i}\left[\lambda^{i}, \lambda^{j}\right]\right) \pi_{j}+\frac{1}{2}\left[\lambda^{i}, \lambda^{j}\right] \pi_{i} \pi_{j} \\
& =\frac{i}{2} \pi_{i}\left(\partial_{j}\left[\lambda^{i}, \lambda^{j}\right]\right)+\frac{1}{2} \pi_{i} \pi_{j}\left[\lambda^{i}, \lambda^{j}\right] .
\end{aligned}
$$

Taking into account that $\left[\lambda^{i}, \lambda^{j}\right]$ is antisymmetric in the indices, one can replace the product $\pi_{i} \pi_{j}$ by $\frac{1}{2}\left[\pi_{i}, \pi_{j}\right]=-\frac{i}{2} \hat{F}_{i j}$, and then replace $\left[\lambda^{i}, \lambda^{j}\right]$ multiplying this expression by $2 \lambda^{i} \lambda^{j}$. Taking now one half of the sum of the two expressions in (36), we find

$$
\frac{1}{2} \pi_{i}\left[\lambda^{i}, \lambda^{j}\right] \pi_{j}=\frac{i}{4}\left[\pi_{j}, \partial_{i}\left[\lambda^{j}, \lambda^{i}\right]\right]_{+}-\frac{i}{2} \lambda^{i} \lambda^{j} \hat{F}_{i j} .
$$

For the next two terms on the rhs of (35) we note

$$
\frac{i}{2}(\partial \cdot \lambda) \lambda^{j} \pi_{j}-\frac{i}{2} \pi_{j} \lambda^{j}(\partial \cdot \lambda)=-\frac{i}{4}\left[\pi_{j},\left[\lambda^{j}, \partial \cdot \lambda\right]\right]_{+}-\frac{1}{4} \partial_{j}\left[\partial \cdot \lambda, \lambda^{j}\right]_{+},
$$

which is shown in a somewhat similar way as the former identity. The sum of the first terms on the rhs of (37) and (38) gives $\frac{i}{4}\left[\pi_{j},\left[\partial_{i} \lambda^{j}, \lambda^{i}\right]\right]_{+}$. In this way we obtain

$$
\begin{aligned}
\tilde{H}^{2}= & \pi_{i} \rho^{i j} \pi_{j}+\frac{i}{4}\left[\partial_{i} \lambda^{j}, \lambda^{i}\right] \pi_{j}+\frac{i}{4} \pi_{j}\left[\partial_{i} \lambda^{j}, \lambda^{i}\right] \\
& -\frac{1}{4} \partial_{j}\left(\left[\lambda^{j}, \partial \cdot \lambda\right]_{+}\right)+\frac{1}{4}(\partial \cdot \lambda)^{2}-\frac{i}{2} \lambda^{i} \lambda^{j} \hat{F}_{i j} .
\end{aligned}
$$

With the use of further notation

$$
\begin{aligned}
& \Lambda_{j}=\frac{i}{4}\left(\rho^{-1}\right)_{j k}\left[\partial_{i} \lambda^{k}, \lambda^{i}\right], \quad \pi_{\Lambda i}=\pi_{i}+\Lambda_{i}, \\
& Q=\frac{1}{4} \partial_{j}\left(\left[\lambda^{j}, \partial \cdot \lambda\right]_{+}\right)-\frac{1}{4}(\partial \cdot \lambda)^{2}, \quad N=Q+\Lambda_{i} \rho^{i j} \Lambda_{j}, \\
& \mathcal{B}=\frac{i}{2} \lambda^{i} \lambda^{j} \hat{F}_{i j},
\end{aligned}
$$

we can write

$$
\tilde{H}^{2}=\pi_{\Lambda} \rho \pi_{\Lambda}-N-\mathcal{B},
$$

where in the first term on the rhs a symbolic notation for summation over indices is used. A straightforward calculation shows that both $Q$ and $N$ are positive numerical functions (times the unit matrix; see Appendix D, formula (96) for $Q$, and then for $N$ this is obvious). Therefore, if we further denote

$$
s=\sqrt{\rho},
$$


then for $\varphi \in \mathcal{D}_{c}\left(\mathbf{p}^{2}\right)$ we have

$$
\|\tilde{H} \varphi\| \leq\left(\left\|s \pi_{\Lambda} \varphi\right\|^{2}-(\varphi, \mathcal{B} \varphi)\right)^{\frac{1}{2}} \leq\left\|s \pi_{\Lambda} \varphi\right\|+|(\varphi, \mathcal{B} \varphi)|^{\frac{1}{2}}
$$

Next denote

$$
X=\frac{1}{2} \mu^{-1} \hat{\mathcal{A}}_{i} \lambda^{i}=\frac{1}{2} \beta a_{i} \lambda^{i}, \quad a_{i}=\left(g^{\tau \tau}\right)^{\frac{1}{2}} \hat{\mathcal{A}}_{i} .
$$

Below we shall need the following identity valid, with our assumptions on $\hat{\mathcal{A}}_{i}$, on $\mathcal{D}_{c}(\mathbf{p})$ :

$$
\beta[X, \tilde{H}]=a_{i} \rho^{i j} \pi_{\Lambda j}-\frac{i}{2} \lambda^{i} \lambda^{j} \partial_{i} a_{j}-\frac{i}{2}\left(\partial_{i} \rho^{i j}\right) a_{j} .
$$

To show this, we note that $[X, \tilde{H}]=\frac{1}{2} \beta\left[\lambda^{i} a_{i}, \tilde{H}\right]_{+}$, write $\tilde{H}=\lambda^{j} \pi_{j}-\frac{i}{2} \partial \cdot \lambda$, and then the lhs of (45) takes the form

$$
\begin{aligned}
& \frac{1}{2} a_{i}\left[\lambda^{i}, \lambda^{j}\right]_{+} \pi_{j}-\frac{i}{2} \lambda^{j} \partial_{j}\left(\lambda^{i} a_{i}\right)-\frac{i}{4} a_{i}\left[\lambda^{i}, \partial_{j} \lambda^{j}\right]_{+} \\
& \quad=a_{i} \rho^{i j} \pi_{\Lambda j}-\frac{i}{2} \lambda^{j} \lambda^{i} \partial_{j} a_{i}-\frac{i}{2} a_{i}\left(\lambda^{j} \partial_{j} \lambda^{i}+\frac{1}{2}\left[\lambda^{i}, \partial_{j} \lambda^{j}\right]_{+}+\frac{1}{2}\left[\partial_{j} \lambda^{i}, \lambda^{j}\right]\right),
\end{aligned}
$$

where after the equality sign we have added and subtracted the term $a_{i} \rho^{i j} \Lambda_{j}$. It is now easy to show that the terms in parentheses multiplying $a_{i}$ sum up to $\frac{1}{2} \partial_{j}\left[\lambda^{j}, \lambda^{i}\right]_{+}$, which ends the proof of $(45)$.

The spreading of the past and future is characterized in our coordinate system by Lemma 15 in Appendix D. Denote

$$
\mathcal{D}\left(r, \mathbf{p}^{2}\right)=\left\{\psi \in \mathcal{D}\left(\mathbf{p}^{2}\right) \mid \psi(\mathbf{z})=0 \text { for }|\mathbf{z}| \geq r\right\},
$$

so that

$$
\mathcal{D}_{c}\left(\mathbf{p}^{2}\right)=\bigcup_{r>0} \mathcal{D}\left(r, \mathbf{p}^{2}\right) .
$$

We set $\tau_{0}=0$ in this lemma, and replace $r_{0}$ and $r$ by $r$ and $r(\tau)$, respectively, so that

$$
r(\tau)=\langle r\rangle|\tau|+r\langle\tau\rangle .
$$

Then, according to this lemma, and statement (C) of Theorem 3, we have

$$
\psi \in \mathcal{D}\left(r, \mathbf{p}^{2}\right) \quad \Longrightarrow \quad U(\tau, 0) \psi \in \mathcal{D}\left(r(\tau), \mathbf{p}^{2}\right) .
$$

For a measurable function $f(\tau, \mathbf{z})$ we define a semi-norm function

$$
\tau \mapsto\|f\|_{r, \tau}=\underset{|\mathbf{z}| \leq r(\tau)}{\operatorname{ess} \sup }|f(\tau, \mathbf{z})|,
$$

and then for $\psi$ as in (46) we find

$$
\|f(\tau, .) U(\tau, 0) \psi\| \leq\|f\|_{r, \tau}\|\psi\| .
$$

Note that according to Lemma 15 we have

$$
|\mathbf{z}| \leq r(\tau) \Longrightarrow\langle z\rangle \leq\langle r\rangle\langle\tau\rangle+r|\tau|,
$$

so in that case

$$
|\mathbf{z}| \leq\langle z\rangle \leq 2\langle r\rangle\langle\tau\rangle
$$

For any measurable function $k(\mathbf{z})$ and $r>0$ we shall denote

$$
\|k\|_{r}=\operatorname{ess} \sup |k(\mathbf{z})| \text {. }
$$

For a function $f(\tau, \mathbf{z})$ we obviously have $\|f(\tau, .)\|_{r} \leq\|f(\tau, .)\|_{r, \tau}$. 
Our scattering theorem will apply to potentials satisfying the following conditions of increasing restrictiveness.

Assumption I. Potential $\hat{\mathcal{A}}_{\mu}(\tau, \mathbf{z})$ satisfies the assumptions of Theorem 6 , and in addition the mapping $\tau \mapsto \hat{\mathcal{A}}_{i}(\tau, \mathbf{z})$ is in $C^{1}\left(\mathbb{R}, L_{\mathrm{loc}}^{\infty}\left(\mathbb{R}^{3}\right)\right)$. For each $r>0$ the following expressions are integrable on $\mathbb{R}$ with respect to $\tau$ :

$$
\left\|\hat{\mathcal{A}}_{\tau}\right\|_{r}, \quad\langle\tau\rangle^{-2}\left\|\hat{\mathcal{A}}_{i}\right\|_{r}^{2}, \quad\langle\tau\rangle^{-2}\left\|\partial_{i} \hat{\mathcal{A}}_{j}\right\|_{r}, \quad\langle\tau\rangle^{-1}\left\|\partial_{\tau} \hat{\mathcal{A}}_{i}\right\|_{r} .
$$

Assumption II. Potential $\hat{\mathcal{A}}_{\mu}(\tau, \mathbf{z})$ satisfies the assumptions of Theorem 6 , and in addition the mapping $\tau \mapsto \hat{\mathcal{A}}_{i}(\tau, \mathbf{z})$ is in $C^{1}\left(\mathbb{R}, L_{\mathrm{loc}}^{\infty}\left(\mathbb{R}^{3}\right)\right)$. For each $r>0$ the following expressions are integrable on $\mathbb{R}$ with respect to $\tau$ :

$$
\begin{aligned}
& \left\|\hat{\mathcal{A}}_{\tau}\right\|_{r, \tau}, \\
& \langle\tau\rangle^{-2}\left(\left\|\hat{\mathcal{A}}_{i}\right\|_{r, \tau}^{2}+\left\|z^{i} \hat{\mathcal{A}}_{i}\right\|_{r, \tau}^{2}\right), \\
& \langle\tau\rangle^{-2}\left(\left\|\partial_{i} \hat{\mathcal{A}}_{j}\right\|_{r, \tau}+\left\|z^{i} \partial_{i} \hat{\mathcal{A}}_{j}\right\|_{r, \tau}+\left\|z^{j} \partial_{i} \hat{\mathcal{A}}_{j}\right\|_{r, \tau}+\left\|z^{i} z^{j} \partial_{i} \hat{\mathcal{A}}_{j}\right\|_{r, \tau}\right), \\
& \langle\tau\rangle^{-1}\left(\left\|\partial_{\tau} \hat{\mathcal{A}}_{i}\right\|_{r, \tau}+\left\|z^{i} \partial_{\tau} \hat{\mathcal{A}}_{i}\right\|_{r, \tau}\right) .
\end{aligned}
$$

Moreover, let $\xi:[1, \infty) \mapsto \mathbb{R}$ be an appropriately chosen smooth, positive, nondecreasing function, such that $\xi(1)=1$ and

$$
\xi^{\prime}(u) \leq \frac{\kappa}{u} \xi(u), \kappa \in\left(0, \frac{1}{2}\right), \quad\left|\xi^{\prime \prime}(u)\right| \leq \frac{\text { const }}{u} \xi(u),
$$

where in the second bound the constant is arbitrary. The following bounds are satisfied

$$
\begin{aligned}
& \left\|\hat{F}_{i j}\right\|_{r, \tau}+\left\|z^{i} \hat{F}_{i j}\right\|_{r, \tau} \leq \operatorname{const}(r) \frac{\langle\tau\rangle}{\xi(\langle\tau\rangle)}, \\
& \left\|\hat{F}_{i \tau}\right\|_{r, \tau}+\left\|z^{i} \hat{F}_{i \tau}\right\|_{r, \tau} \leq \frac{\operatorname{const}(r)}{\xi(\langle\tau\rangle)},
\end{aligned}
$$

and for each $r>0$ the following expressions are integrable on $\mathbb{R}$

$$
\frac{\left\|\xi(\langle z\rangle) \hat{\mathcal{A}}_{i}\right\|_{r, \tau}}{\langle\tau\rangle \xi(\langle\tau\rangle)}, \quad \frac{\left\|\xi(\langle z\rangle) z^{i} \hat{\mathcal{A}}_{i}\right\|_{r, \tau}}{\langle\tau\rangle \xi(\langle\tau\rangle)} .
$$

Before stating the theorem, we make a comment on the function $\xi$ and consider some additional consequences of Assumption II.

If one sets $\xi(u)=u^{\kappa} \xi_{0}(u)$, then the first bound in (48) is equivalent to $\xi_{0}^{\prime}(u) \leq 0$. It follows that

$$
\xi(u) \leq u^{\kappa}
$$

Therefore, $\xi$ is a slowly increasing, non-oscillating function. Examples include:

$$
\begin{aligned}
& \xi_{1}(u)=u^{\kappa}, \\
& \xi_{2}(u)=\left[1+\frac{\kappa}{m} \log (u)\right]^{m}, \quad m>0,
\end{aligned}
$$

where $\kappa$ is as assumed in (48). A particular choice of $\xi$ must guarantee the validity of the assumptions (if this is possible). 
By the Schwarz inequality, also the following integral is finite:

$$
\int_{\mathbb{R}} \frac{\left\|\hat{\mathcal{A}}_{i}\right\|_{r, \tau}}{\langle\tau\rangle^{2}} \mathrm{~d} \tau \leq \sqrt{\pi}\left(\int_{\mathbb{R}} \frac{\left\|\hat{\mathcal{A}}_{i}\right\|_{r, \tau}^{2}}{\langle\tau\rangle^{2}} d \tau\right)^{\frac{1}{2}}<\infty
$$

and similarly for $z^{i} \hat{\mathcal{A}}_{i}$. Moreover, for $\tau_{2} \geq \tau_{1}$ we have

$$
\frac{\hat{\mathcal{A}}_{i}\left(\tau_{2}, \mathbf{z}\right)}{\left\langle\tau_{2}\right\rangle}-\frac{\hat{\mathcal{A}}_{i}\left(\tau_{1}, \mathbf{z}\right)}{\left\langle\tau_{1}\right\rangle}=\int_{\tau_{1}}^{\tau_{2}}\left(\frac{\partial_{\sigma} \hat{\mathcal{A}}_{i}(\sigma, \mathbf{z})}{\langle\sigma\rangle}-\frac{\sigma \hat{\mathcal{A}}_{i}(\sigma, \mathbf{z})}{\langle\sigma\rangle^{3}}\right) \mathrm{d} \sigma,
$$

and similarly for $z^{i} \hat{\mathcal{A}}_{i}$. Therefore, by Assumption II the functions

$$
\langle\tau\rangle^{-1}\left\|\hat{\mathcal{A}}_{i}\right\|_{r, \tau} \quad \text { and } \quad\langle\tau\rangle^{-1}\left\|z^{i} \hat{\mathcal{A}}_{i}\right\|_{r, \tau}
$$

have limits for $\tau \rightarrow \pm \infty$. Limits different from zero would contradict other assumptions, so

$$
\lim _{\tau \rightarrow \pm \infty} \frac{\left\|\hat{\mathcal{A}}_{i}\right\|_{r, \tau}}{\langle\tau\rangle}=\lim _{\tau \rightarrow \pm \infty} \frac{\left\|z^{i} \hat{\mathcal{A}}_{i}\right\|_{r, \tau}}{\langle\tau\rangle}=0 .
$$

For the sake of the proof of the coming theorem, we note the following estimates easily obtained with the use of formula (89):

$$
\begin{aligned}
& \left|\lambda^{i} \hat{\mathcal{A}}_{i}\right| \leq \frac{\text { const }}{\langle\tau\rangle}\left(\frac{\langle\tau \mid z\rangle}{\langle\tau\rangle}\left|\hat{\mathcal{A}}_{i}\right|+\left|z^{i} \hat{\mathcal{A}}_{i}\right|\right) \\
& \left|\lambda^{i} \lambda^{j} \partial_{i} \hat{\mathcal{A}}_{j}\right| \leq \frac{\mathrm{const}}{\langle\tau\rangle^{2}}\left[\frac{\langle\tau \mid z\rangle^{2}}{\langle\tau\rangle^{2}}\left|\partial_{i} \hat{\mathcal{A}}_{j}\right|+\frac{\langle\tau \mid z\rangle}{\langle\tau\rangle}\left(\left|z^{i} \partial_{i} \hat{\mathcal{A}}_{j}\right|+\left|z^{j} \partial_{i} \hat{\mathcal{A}}_{j}\right|\right)+\left|z^{i} z^{j} \partial_{i} \hat{\mathcal{A}}_{j}\right|\right] .
\end{aligned}
$$

Theorem 7. (i) Let $\hat{\mathcal{A}}_{\mu}$ satisfy Assumption I. Then the following strong limits exist:

$$
\begin{aligned}
& \underset{\tau \rightarrow \pm \infty}{\mathrm{s}-\lim _{\tau \rightarrow \pm}} U(0, \tau) U_{0}(\tau, 0)=\Omega_{\mp}, \\
& \mathrm{s}-\lim _{\tau \rightarrow \pm \infty} U_{\Phi}(0, \tau)=U_{\Phi}(0, \pm \infty)=\Omega_{\mp} U_{0 \Phi}(0, \pm \infty) .
\end{aligned}
$$

(ii) Let $\hat{\mathcal{A}}_{\mu}$ satisfy Assumption II. Then the operators $\Omega_{\mp}$ are unitary and also the following strong limits exist:

$$
\begin{aligned}
& \underset{\tau \rightarrow \pm \infty}{\mathrm{s}-\lim _{\tau}} U_{0}(0, \tau) U(\tau, 0)=\Omega_{\mp}^{*}, \\
& \underset{\tau \rightarrow \pm \infty}{\mathrm{s}-\lim _{\tau}} U_{\Phi}(\tau, 0)=U_{\Phi}( \pm \infty, 0)=U_{0 \Phi}( \pm \infty, 0) \Omega_{\mp}^{*} .
\end{aligned}
$$

Proof. For the sake of the whole proof we assume that $\psi \in \mathcal{D}\left(r, \mathbf{p}^{2}\right)$ and $\|\psi\|=1$.

(i) For $\tau \rightarrow \infty$, we prove the existence of the limit (53), from which the limit (52) follows with the use of Theorem 5 . The case $\tau \rightarrow-\infty$ is analogous.

We note the identity

$$
\left(1-\frac{1}{2} \beta \tilde{H}\right) \beta-H\left(1-\frac{1}{2} \beta \tilde{H}\right)=\left(\mu-\beta+\hat{\mathcal{A}}_{\tau}\right)\left(\frac{1}{2} \beta \tilde{H}-1\right)-\frac{1}{2} \beta \tilde{H}^{2},
$$


where $\tilde{H}$ is as defined in (33) (to show this one eliminates $H$ with the use of (33) and takes into account the anticommutation relation (34)). Using it, we obtain the evolution equation

$$
\begin{aligned}
& i \partial_{\tau} {\left[U(0, \tau)\left(1-\frac{1}{2} \beta \tilde{H}\right) \Phi(\tau)\right] \psi } \\
& \quad=U(0, \tau)\left[-\frac{i}{2} \beta\left(\partial_{\tau} \tilde{H}\right)+\left(\mu-\beta+\hat{\mathcal{A}}_{\tau}\right)\left(\frac{1}{2} \beta \tilde{H}-1\right)-\frac{1}{2} \beta \tilde{H}^{2}\right] \Phi(\tau) \psi .
\end{aligned}
$$

Taking into account the anticommutation relation (34), we can write this in the form

$$
\begin{aligned}
i \partial_{\tau}[ & \left.U_{\Phi}(0, \tau)-\frac{1}{2} U(0, \tau) \Phi(\tau)^{*} \beta \tilde{H}\right] \psi \\
= & -U(0, \tau) \Phi(\tau)\left[\mu-\beta+\hat{\mathcal{A}}_{\tau}+\frac{1}{2} \beta \tilde{H}^{2}\right] \psi \\
& \quad+\frac{1}{2} U(0, \tau) \Phi(\tau)^{*} \beta\left[-i \dot{\tilde{H}}+\left(\mu-\beta+\hat{\mathcal{A}}_{\tau}\right) \tilde{H}\right] \psi .
\end{aligned}
$$

We estimate the terms in this equation, starting with the second term in brackets on the lhs, and then going to the successive terms on the rhs. As $|\mathbf{z}| \leq r$ on the support of $\psi$, each $\lambda^{i}, \partial_{i} \lambda^{j}$ and $\partial_{i} \partial_{j} \lambda^{k}$ give a bounding factor const $(r)\langle\tau\rangle^{-1}$, and each $\partial_{\tau} \lambda^{i}$ a factor const $(r)\langle\tau\rangle^{-2}$, which leads to an easy straightforward estimation:

$$
\begin{aligned}
& \|\tilde{H} \psi\| \leq \frac{\operatorname{const}(r)}{\langle\tau\rangle}\left(\left\|p_{i} \psi\right\|+\left\|\hat{\mathcal{A}}_{i}\right\|_{r}+1\right), \\
& \|(\mu-\beta) \psi\| \leq \frac{r^{2}}{2\langle\tau\rangle^{2}}, \quad\left\|\hat{\mathcal{A}}_{\tau} \psi\right\| \leq\left\|\hat{\mathcal{A}}_{\tau}\right\|_{r}, \\
& \left\|\tilde{H}^{2} \psi\right\| \leq \frac{\operatorname{const}(r)}{\langle\tau\rangle^{2}}\left[\left\|p_{i} p_{j} \psi\right\|+\left(\left\|\hat{\mathcal{A}}_{i}\right\|_{r}+1\right)\left(\left\|\hat{\mathcal{A}}_{i}\right\|_{r}+1+\left\|p_{i} \psi\right\|\right)\right], \\
& \|\dot{\tilde{H}} \psi\| \leq \frac{\operatorname{const}(r)}{\langle\tau\rangle^{2}}\left(\left\|p_{i} \psi\right\|+\left\|\hat{\mathcal{A}}_{i}\right\|_{r}+1\right)+\frac{\operatorname{const}(r)}{\langle\tau\rangle}\left\|\partial_{\tau} \hat{\mathcal{A}}_{i}\right\|_{r}, \\
& \left\|\left(\mu-\beta+\hat{\mathcal{A}}_{\tau}\right) \tilde{H} \psi\right\| \leq \frac{\operatorname{const}(r)}{\langle\tau\rangle}\left(\frac{r^{2}}{\langle\tau\rangle^{2}}+\left\|\hat{\mathcal{A}}_{\tau}\right\|_{r}\right)\left(\left\|p_{i} \psi\right\|+\left\|\hat{\mathcal{A}}_{i}\right\|_{r}+1\right) .
\end{aligned}
$$

Therefore, with the conditions of Assumption I all the terms on the rhs of (56) are integrable on $\mathbb{R}$, so the strong limit of $U_{\Phi}(0, \tau) \psi-\frac{1}{2} U(0, \tau) \Phi(\tau)^{*} \beta \tilde{H} \psi$ exists. But the second term vanishes in the limit, so the thesis follows for $\psi \in \mathcal{D}_{c}\left(\mathbf{p}^{2}\right)$, and then by isometry for all $\psi \in \mathcal{H}$. (For the integrability of $\langle\tau\rangle^{-2}\left\|\hat{\mathcal{A}}_{i}\right\|_{r}$ and for vanishing of $\langle\tau\rangle^{-1}\left\|\hat{\mathcal{A}}_{i}\right\|_{r}$ one argues similarly as in the remarks following Assumption II.)

(ii) We prove the existence of the limit (54) for $\tau \rightarrow \infty$, from which the limit (55) follows. Combined with the existence of the limit (52), this also leads to unitarity and the conjugation relation. The case $\tau \rightarrow-\infty$ is similar.

We note the identity

$$
(1+X) H-H_{0}(1+X)=\hat{\mathcal{A}}_{\tau}+W X+[X, \tilde{H}],
$$

where $W$ and $X$ are defined in (28) and (44), respectively, and we used the fact that

$$
[X, H-\tilde{H}]=[X, \mu]=-2 \mu X=-\hat{\mathcal{A}}_{i} \lambda^{i}
$$


Thus

$$
\begin{aligned}
& i \partial_{\tau} {\left[U_{0}(0, \tau)(1+X) U(\tau, 0)\right] \psi } \\
& \quad=U_{0}(0, \tau)\left(i \partial_{\tau} X+\hat{\mathcal{A}}_{\tau}+W X+[X, \tilde{H}]\right) U(\tau, 0) \psi .
\end{aligned}
$$

If we can show that the norm of the rhs of (57) is integrable over $[0,+\infty)$, then the strong limit of $U_{0}(0, \tau)(1+X) U(\tau, 0) \psi$ for $\tau \rightarrow \infty$ exists. But with the use of formula (47), and taking into account (50) and the value of $g^{\tau \tau}$ to be found in Appendix D, we obtain

$$
\|X(\tau) U(\tau, 0) \psi\| \leq\|X\|_{r, \tau} \leq \frac{\text { const }}{\langle\tau\rangle}\left(\left\|\hat{\mathcal{A}}_{i}\right\|_{r, \tau}+\left\|z^{i} \hat{\mathcal{A}}_{i}\right\|_{r, \tau}\right)
$$

so this norm vanishes in the limit, which then implies the desired result.

We estimate the norms of the successive terms on the rhs of (57), again with the use of (47). Differentiating formula (90) with respect to $\tau$, we find

$$
\begin{aligned}
\left\|\left(\partial_{\tau} X\right) U(\tau, 0) \psi\right\| \leq & \left\|\partial_{\tau} X\right\|_{r, \tau} \leq \frac{\mathrm{const}}{\langle\tau\rangle^{2}}\left(\left\|\hat{\mathcal{A}}_{i}\right\|_{r, \tau}+\left\|z^{i} \hat{\mathcal{A}}_{i}\right\|_{r, \tau}\right) \\
& +\frac{\mathrm{const}}{\langle\tau\rangle}\left(\left\|\partial_{\tau} \hat{\mathcal{A}}_{i}\right\|_{r, \tau}+\left\|z^{i} \partial_{\tau} \hat{\mathcal{A}}_{i}\right\|_{r, \tau}\right)
\end{aligned}
$$

which is integrable. The norm of the second term is bounded by $\left\|\hat{\mathcal{A}}_{\tau}(\tau)\right\|_{r, \tau}$, which is integrable by assumption. Next we note that

$$
\beta W X=\hat{\mathcal{A}}_{\tau} \beta X-\frac{1}{2}\left(g^{\tau \tau}\right)^{\frac{1}{2}} \hat{\mathcal{A}}_{i} \rho^{i j} \hat{\mathcal{A}}_{j},
$$

so using the explicit form of $\left(g^{\tau \tau}\right)^{\frac{1}{2}} \rho^{i j}$, see (91), we estimate the norm of the third term on the rhs of (57) by

$$
\|W X\|_{r, \tau} \leq \mathrm{const}\left\|\hat{\mathcal{A}}_{\tau}\right\|_{r, \tau}\|X\|_{r, \tau}+\frac{\operatorname{const}\langle r\rangle}{\langle\tau\rangle^{2}}\left(\left\|\hat{\mathcal{A}}_{i}\right\|_{r, \tau}^{2}+\left\|z^{i} \hat{\mathcal{A}}_{i}\right\|_{r, \tau}^{2}\right),
$$

which ensures integrability.

To estimate the norm of the fourth term, we use (45), (51) and (93) to find

$$
\begin{aligned}
& \left\|\lambda^{i} \lambda^{j} \partial_{i} a_{j}+\left(\partial_{i} \rho^{i j}\right) a_{j}\right\|_{r, \tau} \leq \mathrm{const} \frac{\langle r\rangle}{\langle\tau\rangle^{2}}\left\|\partial_{i} \hat{\mathcal{A}}_{j}\right\|_{r, \tau} \\
& \quad+\frac{\text { const }}{\langle\tau\rangle^{2}}\left(\left\|\hat{\mathcal{A}}_{i}\right\|_{r, \tau}+\left\|z^{i} \hat{\mathcal{A}}_{i}\right\|_{r, \tau}+\left\|z^{i} \partial_{i} \hat{\mathcal{A}}_{j}\right\|_{r, \tau}+\left\|z^{j} \partial_{i} \hat{\mathcal{A}}_{j}\right\|_{r, \tau}+\left\|z^{i} z^{j} \partial_{i} \hat{\mathcal{A}}_{j}\right\|_{r, \tau}\right),
\end{aligned}
$$

which again is integrable.

We are now left with the single term $a \rho \pi_{\Lambda} U(\tau, 0) \psi$. As it turns out, in this case the methods applied up to now are insufficient and it is here that we make use of the function $\xi$. We write $\psi_{\tau}=U(\tau, 0) \psi$ and note that

$$
\begin{aligned}
\left\|a \rho \pi_{\Lambda} \psi_{\tau}\right\| & \leq\|s a \xi(\langle z\rangle)\|_{r, \tau}\left\|\xi(\langle z\rangle)^{-1} s \pi_{\Lambda} \psi_{\tau}\right\| \\
& \leq\langle\tau\rangle^{-1}\left(\left\|\xi(\langle z\rangle) \hat{\mathcal{A}}_{i}\right\|_{r, \tau}+\left\|\xi(\langle z\rangle) z^{i} \hat{\mathcal{A}}_{i}\right\|_{r, \tau}\right)\left\|\xi(\langle z\rangle)^{-1} s \pi_{\Lambda} \psi_{\tau}\right\| .
\end{aligned}
$$

Now, the estimation of $\left\|\xi(\langle z\rangle)^{-1} s \pi_{\Lambda} \psi_{\tau}\right\|$ is the most difficult part of the proof and we shift it to the lemma below. Substituting its result in the above estimate 
and using Assumption II, one completes the proof of the existence of the limit $\tau \rightarrow+\infty$.

Lemma 8. Under the conditions of Assumption II, for $\psi \in \mathcal{D}_{c}\left(\mathbf{p}^{2}\right)$ the following estimate holds

$$
\left\|\xi(\langle z\rangle)^{-1} s \pi_{\Lambda} U(\tau, 0) \psi\right\| \leq \operatorname{const}(\psi) \xi(\langle\tau\rangle)^{-1} .
$$

Proof. We assume again that $\psi \in \mathcal{D}\left(r, \mathbf{p}^{2}\right)$ and $\|\psi\|=1$. We observe that the lhs of the inequality may be equivalently replaced by $\left\|s \pi_{\Lambda} \xi(\langle z\rangle)^{-1} U(\tau, 0) \psi\right\|$. Indeed, we have

$$
\left\|\left[s \pi_{\Lambda}, \xi(\langle z\rangle)^{-1}\right]\right\|_{\infty}=\left\|\frac{\mathbf{z} \xi^{\prime}(\langle z\rangle)}{\langle\tau\rangle \xi(\langle z\rangle)^{2}}\right\|_{\infty} \leq\left\|\frac{\kappa \mathbf{z}}{\langle\tau\rangle\langle z\rangle \xi(\langle z\rangle)}\right\|_{\infty} \leq \frac{\kappa}{\langle\tau\rangle} .
$$

Now we shall estimate the norm squared

$$
\left\|s \pi_{\Lambda} \xi(\langle z\rangle)^{-1} \psi_{\tau}\right\|^{2}=\left(\pi_{\Lambda} \xi(\langle z\rangle)^{-1} \psi_{\tau}, \rho \pi_{\Lambda} \xi(\langle z\rangle)^{-1} \psi_{\tau}\right)
$$

by first finding a differential inequality, and then integrating. Preparing for that, we denote

$$
\nu=i \xi(\langle z\rangle)^{-1}[H, \xi(\langle z\rangle)]=\frac{\lambda^{i} \partial_{i} \xi(\langle z\rangle)}{\xi(\langle z\rangle)}=\frac{\xi^{\prime}(\langle z\rangle)}{\langle\tau\rangle \xi(\langle z\rangle)} z^{i} \alpha^{i},
$$

with the standard notation $\alpha^{i}=\beta \gamma^{i}$, and observe that

$$
\xi(\langle z\rangle)^{-1} H=\tilde{H} \xi(\langle z\rangle)^{-1}+\left(\hat{\mathcal{A}}_{\tau}+\mu-i \nu\right) \xi(\langle z\rangle)^{-1} .
$$

To shorten notation, we shall write $\psi_{\tau}^{\xi}=\xi(\langle z\rangle)^{-1} \psi_{\tau}$. Looking at the explicit form of $\Lambda_{i}(94)$, we note that

$$
\left[\Lambda_{i}, \mu\right]=0, \quad\left[\Lambda_{i}, \nu\right]_{+}=0 .
$$

Now calculate

$$
\begin{aligned}
\partial_{\tau}\left[\pi_{\Lambda} \psi_{\tau}^{\xi}\right] & =-i \pi_{\Lambda} \xi(\langle z\rangle)^{-1} H \psi_{\tau}+\left(\partial_{\tau} \Lambda+\partial_{\tau} \hat{\mathcal{A}}\right) \psi_{\tau}^{\xi} \\
& =-i \pi_{\Lambda} \tilde{H} \psi_{\tau}^{\xi}+\left(\dot{\Lambda}+\hat{F}_{\tau .}+\partial(i \nu-\mu)+2 \nu \Lambda\right) \psi_{\tau}^{\xi}-\left(\nu+i \hat{\mathcal{A}}_{\tau}+i \mu\right) \pi_{\Lambda} \psi_{\tau}^{\xi}
\end{aligned}
$$

and

$$
\begin{aligned}
& \partial_{\tau}\left(\pi_{\Lambda} \psi_{\tau}^{\xi}, \rho \pi_{\Lambda} \psi_{\tau}^{\xi}\right)-\left(\pi_{\Lambda} \psi_{\tau}^{\xi}, \dot{\rho} \pi_{\Lambda} \psi_{\tau}^{\xi}\right)=-2\left(\pi_{\Lambda} \psi_{\tau}^{\xi}, \rho \nu \pi_{\Lambda} \psi_{\tau}^{\xi}\right) \\
& \quad-i\left(\pi_{\Lambda} \rho \pi_{\Lambda} \psi_{\tau}^{\xi}, \tilde{H} \psi_{\tau}^{\xi}\right)+i\left(\tilde{H} \psi_{\tau}^{\xi}, \pi_{\Lambda} \rho \pi_{\Lambda} \psi_{\tau}^{\xi}\right) \\
& \quad+2 \operatorname{Re}\left(\pi_{\Lambda} \psi_{\tau}^{\xi}, \rho\left[\dot{\Lambda}+\hat{F}_{\tau}+\partial(i \nu-\mu)+2 \nu \Lambda\right] \psi_{\tau}^{\xi}\right)
\end{aligned}
$$

where in both identities $\hat{F}_{\tau}$. denotes $\hat{F}_{\tau i}$ with the index $i$ suppressed (in the second identity summation over this index is implied). In the first identity we have used (60) and commuted $\pi_{\Lambda}$ with the term $\left(\hat{\mathcal{A}}_{\tau}+\mu-i \nu\right)$.

From now on we continue the proof for $\tau \geq 0$; for $\tau \leq 0$ the proof is analogous, but equation (61) has to be multiplied by -1 before continuing. The first term on the rhs of (61) is bounded in absolute value by

$$
2\|\nu\|_{\infty}\left(\pi_{\Lambda} \psi_{\tau}^{\xi}, \rho \pi_{\Lambda} \psi_{\tau}^{\xi}\right) \leq 2 \kappa\langle\tau\rangle^{-1}\left\|s \pi_{\Lambda} \psi_{\tau}^{\xi}\right\|^{2},
$$


where we used the estimate given in (99). With the use of formula (41), the second line of equation (61) takes the form $2 \operatorname{Re} i\left(\tilde{H} \psi_{\tau}^{\xi},(N+\mathcal{B}) \psi_{\tau}^{\xi}\right)$, and thanks to the estimate (43) is bounded in absolute value by

$$
2\left(\left\|s \pi_{\Lambda} \psi_{\tau}^{\xi}\right\|+\left|\left(\psi_{\tau}^{\xi}, \mathcal{B} \psi_{\tau}^{\xi}\right)\right|^{\frac{1}{2}}\right)\left\|(N+\mathcal{B}) \psi_{\tau}^{\xi}\right\|
$$

The third line in (61) is bounded by

$$
2\left\|s \pi_{\Lambda} \psi_{\tau}^{\xi}\right\|\left\|s\left(\dot{\Lambda}+\hat{F}_{\tau} .+\partial(i \nu-\mu)+2 \nu \Lambda\right) \psi_{\tau}^{\xi}\right\| .
$$

Finally, we observe that for $\tau>0$ we have [see (92)]

$$
\dot{\rho} \leq-\frac{2 \tau}{\langle\tau\rangle^{2}} \rho,
$$

which allows us to use (61) for the following estimate:

$$
\partial_{\tau}\left\|s \pi_{\Lambda} \psi_{\tau}^{\xi}\right\|^{2} \leq-b\left\|s \pi_{\Lambda} \psi_{\tau}^{\xi}\right\|^{2}+2 c\left\|s \pi_{\Lambda} \psi_{\tau}^{\xi}\right\|+d,
$$

where

$$
\begin{aligned}
& b=\frac{2 \tau}{\langle\tau\rangle^{2}}-\frac{2 \kappa}{\langle\tau\rangle}, \\
& c=\left\|(N+\mathcal{B}) \psi_{\tau}^{\xi}\right\|+\left\|s\left(\dot{\Lambda}+\hat{F}_{\tau} .+\partial(i \nu-\mu)+2 \nu \Lambda\right) \psi_{\tau}^{\xi}\right\|, \\
& d=2\left|\left(\psi_{\tau}^{\xi}, \mathcal{B} \psi_{\tau}^{\xi}\right)\right|^{\frac{1}{2}}\left\|(N+\mathcal{B}) \psi_{\tau}^{\xi}\right\| .
\end{aligned}
$$

The second term on the rhs of (62) may be estimated as follows

$$
\begin{aligned}
2 c\left\|s \pi_{\Lambda} \psi_{\tau}^{\xi}\right\| & =2\left[\frac{1-2 \kappa}{\langle\tau\rangle}\right]^{\frac{1}{2}}\left\|s \pi_{\Lambda} \psi_{\tau}^{\xi}\right\|\left[\frac{\langle\tau\rangle}{1-2 \kappa}\right]^{\frac{1}{2}} c \\
& \leq \frac{1-2 \kappa}{\langle\tau\rangle}\left\|s \pi_{\Lambda} \psi_{\tau}^{\xi}\right\|^{2}+\frac{\langle\tau\rangle c^{2}}{1-2 \kappa},
\end{aligned}
$$

which results in the inequality

$$
\partial_{\tau}\left\|s \pi_{\Lambda} \psi_{\tau}^{\xi}\right\|^{2} \leq-b_{0}\left\|s \pi_{\Lambda} \psi_{\tau}^{\xi}\right\|^{2}+d_{0},
$$

where

$$
b_{0}=\frac{2 \tau}{\langle\tau\rangle^{2}}-\frac{1}{\langle\tau\rangle}, \quad d_{0}=\frac{\langle\tau\rangle c^{2}}{1-2 \kappa}+d
$$

We set

$$
\left\|s \pi_{\Lambda} \psi_{\tau}^{\xi}\right\|^{2}=\exp \left(-\int_{0}^{\tau} b_{0}(\sigma) \mathrm{d} \sigma\right) f(\tau)=\frac{\tau+\langle\tau\rangle}{\langle\tau\rangle^{2}} f(\tau)
$$

and then (64) takes the form

$$
\partial_{\tau} f \leq \frac{\langle\tau\rangle^{2}}{\tau+\langle\tau\rangle} d_{0}(\tau) \leq\langle\tau\rangle d_{0}(\tau) .
$$

We note that $f(0)=\left\|\left[s \pi_{\Lambda} \psi_{\tau}^{\xi}\right]_{\tau=0}\right\|^{2}$ and find

$$
\left\|s \pi_{\Lambda} \psi_{\tau}^{\xi}\right\|^{2} \leq \frac{2}{\langle\tau\rangle}\left[\left\|\left[s \pi_{\Lambda} \psi_{\tau}^{\xi}\right]_{\tau=0}\right\|^{2}+\int_{0}^{\tau}\langle\sigma\rangle d_{0}(\sigma) \mathrm{d} \sigma\right] .
$$


We have to estimate $d_{0}$ defined in (65). We start by estimating $c$. For the terms depending on the electromagnetic field, we have (we use the form of $s$ and estimates of $\lambda^{i}$ given in Appendix D)

$$
\begin{gathered}
\|\mathcal{B}\|_{r, \tau} \leq \frac{\operatorname{const}\langle r\rangle^{2}}{\langle\tau\rangle^{2}}\left(\left\|\hat{F}_{i j}\right\|_{r, \tau}+\left\|z^{i} \hat{F}_{i j}\right\|_{r, \tau}\right) \leq \frac{\operatorname{const}(r)}{\langle\tau\rangle \xi(\langle\tau\rangle)}, \\
\left\|s \hat{F}_{\tau .}\right\|_{r, \tau} \leq \frac{\operatorname{const}\langle r\rangle}{\langle\tau\rangle}\left(\left\|\hat{F}_{\tau i}\right\|_{r, \tau}+\left\|z^{i} \hat{F}_{\tau i}\right\|_{r, \tau}\right) \leq \frac{\operatorname{const}(r)}{\langle\tau\rangle \xi(\langle\tau\rangle)} .
\end{gathered}
$$

For the term $s^{i j} \partial_{j}(i \nu-\mu)$, using the estimates (98) and (100) in Appendix D we find ${ }^{9}$

$$
\left\|s \partial(i \nu-\mu) \psi_{\tau}^{\xi}\right\| \leq\left\|\frac{s \partial(i \nu-\mu)}{\xi(\langle z\rangle)}\right\|_{r, \tau} \leq \frac{\mathrm{const}}{\langle\tau\rangle^{2}}\left\|\frac{\langle z\rangle}{\xi(\langle z\rangle)}\right\|_{r, \tau} \leq \frac{\operatorname{const}(r)}{\langle\tau\rangle \xi(\langle\tau\rangle)},
$$

where we used the fact, that both $u / \xi(u)$ as well as $\xi(u)$ are increasing, so

$$
\frac{\langle z\rangle}{\xi(\langle z\rangle)} \leq \frac{2\langle r\rangle\langle\tau\rangle}{\xi(2\langle r\rangle\langle\tau\rangle)} \leq 2\langle r\rangle \frac{\langle\tau\rangle}{\xi(\langle\tau\rangle)} .
$$

The estimation of the other terms in (63) uses the bounds (95), (97) and (99) and gives

$$
\left\|N \psi_{\tau}^{\xi}\right\|+\left\|s \dot{\Lambda} \psi_{\tau}^{\xi}\right\|+\left\|2 \nu \Lambda \psi_{\tau}^{\xi}\right\| \leq \frac{\mathrm{const}}{\langle\tau\rangle^{2}}
$$

so summing up, we have

$$
c \leq \frac{\operatorname{const}(r)}{\langle\tau\rangle \xi(\langle\tau\rangle)}, \quad\langle\tau\rangle c^{2} \leq \frac{\operatorname{const}(r)}{\langle\tau\rangle \xi(\langle\tau\rangle)^{2}} .
$$

The use of (67) and (69) shows that also

$$
d(\tau) \leq \frac{\operatorname{const}(r)}{[\langle\tau\rangle \xi(\langle\tau\rangle)]^{\frac{3}{2}}} \leq \frac{\operatorname{const}(r)}{\langle\tau\rangle \xi(\langle\tau\rangle)^{2}}
$$

where we used the bound $u^{\frac{1}{2}} \geq \xi(u) \geq 1$, see (49). Summing up, we obtain

$$
d_{0}(\tau) \leq \frac{\operatorname{const}(r)}{\langle\tau\rangle \xi(\langle\tau\rangle)^{2}}
$$

Now, it follows from (48) that $u^{\kappa} / \xi(u)$ is an increasing function. Therefore,

$$
\begin{aligned}
\int_{0}^{\tau}\langle\sigma\rangle d_{0}(\sigma) \mathrm{d} \sigma & \leq \operatorname{const}(r) \int_{0}^{\tau} \frac{\mathrm{d} \sigma}{\xi(\langle\sigma\rangle)^{2}} \leq \operatorname{const}(r) \frac{\langle\tau\rangle^{2 \kappa}}{\xi(\langle\tau\rangle)^{2}} \int_{0}^{\tau} \frac{\mathrm{d} \sigma}{\langle\sigma\rangle^{2 \kappa}} \\
& \leq \operatorname{const}(r) \frac{\langle\tau\rangle}{\xi(\langle\tau\rangle)^{2}} .
\end{aligned}
$$

This, when used in (66), gives

$$
\left\|s \pi_{\Lambda} \psi_{\tau}^{\xi}\right\|^{2} \leq \frac{2\left\|\left[s \pi_{\Lambda} \psi_{\tau}^{\xi}\right]_{\tau=0}\right\|^{2}}{\langle\tau\rangle}+\frac{\operatorname{const}(r)}{\xi(\langle\tau\rangle)^{2}} \leq \frac{\operatorname{const}(\psi)}{\xi(\langle\tau\rangle)^{2}},
$$

where for the second inequality we used (49).

\footnotetext{
${ }^{9}$ The problem of estimation of the term $s^{i j} \partial_{j} \mu$ is the ultimate reason for our introduction of the function $\xi$. Without it, the bound in (68) would have the form $\operatorname{const}(r)\langle\tau\rangle^{-1}$, which would be insufficient for our application.
} 


\section{Typical Electromagnetic Field and Its Special Gauges}

Assumption II, on which our main theorem on scattering 7 is based, is rather technical and not easy for interpretation. Here we formulate a rather typical situation met in scattering processes. ${ }^{10}$ We show that the electromagnetic field thus identified admits a gauge in which Assumption II is satisfied. Moreover, there is a class of gauges which need not satisfy this assumption, but still assure a similar asymptotic structure.

The retarded and advanced potentials are defined in terms of the source current $J$ in standard way (as $\varphi_{\text {ret/adv }}$ is defined in terms of $\rho$ in (118) in Appendix G). Also, the radiated field of the current $J$ is defined in standard way, $A_{\text {rad }}=A_{\text {ret }}-A_{\text {adv }}$. The Heaviside step function is denoted by $\theta$.

Assumption III. The Lorenz potential $A_{a}$ of the electromagnetic field $F_{a b}$ is given by

$$
A=A_{\text {ret }}+A_{\text {in }}=A_{\text {adv }}+A_{\text {out }},
$$

where $A_{\text {ret }}$ is the retarded potential of a current $J$ satisfying the assumptions listed below, and $A_{\text {in }}$ is the radiated potential of another current $J_{\text {in }}$ with similar properties as $J$. Then also $A_{\mathrm{adv}}$ is the advanced potential of the current $J$, and $A_{\text {out }}$ is the radiated potential of the current $J_{\text {out }}=J+J_{\text {in }}$, which has similar properties as $J$ and $J_{\text {in }}$.

The conserved current $J(x)$ is of class $C^{3}$ and for some $0<\varepsilon<\frac{1}{2}$ satisfies the following estimates:

$$
\begin{array}{ll}
\left|\nabla^{\alpha} J(x)\right| \leq \frac{1}{(|x|+1)^{3+|\alpha|}}\left[\theta\left(x^{2}\right)+\frac{1}{(|x|+1)^{\varepsilon}}\right], & \text { for } \quad|\alpha| \leq 3, \\
\left|\nabla^{\alpha}(x \cdot \nabla+3) J(x)\right| \leq \frac{\text { const }}{(|x|+1)^{3+|\alpha|+\varepsilon},} & \text { for } \quad|\alpha| \leq 2 .
\end{array}
$$

The same is assumed for $J_{\text {in }}$, and then the same follows for $J_{\text {out }}$. The potential $A$ is then of class $C^{3}$ on the Minkowski spacetime.

Note that $A$ is a linear combination of retarded and advanced potentials of currents satisfying (70). Therefore, the last statement of Assumption III is a consequence of Lemma 20 (i) in Appendix G.

Theorem 9. Let the electromagnetic field $F$ and its Lorenz potential A satisfy Assumption III. Define a new gauge by

$$
\mathcal{A}(x)=A(x)-\nabla S(x) .
$$

Then the following holds.

(i) For the choice

$$
S(x)=S_{\ell}(x) \equiv \log (\langle\tau\rangle\langle z\rangle) x \cdot A(x),
$$

the potential (72) fulfills Assumption II, so Theorems 6 and 7 are satisfied.

\footnotetext{
${ }^{10}$ Possible oscillating terms in the asymptotic behavior of charged currents are not taken into account. One can expect that fields produced by such terms decay more rapidly than those considered here; see also Discussion.
} 
(ii) Let $S(x)$ be another gauge function, such that the difference

$$
\mathcal{G}(x)=S(x)-S_{\ell}(x)
$$

satisfies the assumptions of Theorem 6 (ii), so that the thesis of this theorem is true. Suppose, in addition, that there exist point-wise limits

$$
\lim _{\tau \rightarrow \pm \infty} \mathcal{G}(\tau, \mathbf{z}) \equiv \mathcal{G}_{ \pm}(\mathbf{z}) .
$$

Then the potential (72) satisfies the thesis of Theorem 7 (but not necessarily the assumption of this theorem).

(iii) In particular, the gauges defined by:

$$
S_{\log }(x)=\log \langle\tau\rangle x \cdot A(x),
$$

$$
S_{\mathrm{tr}}(x)=\int_{0}^{\tau} \partial_{\sigma} x(\sigma, \mathbf{z}) \cdot A(\sigma, \mathbf{z}) \mathrm{d} \sigma,
$$

are in the class defined in (ii). In case (b) one has $\hat{\mathcal{A}}_{\tau}=0$ and

$$
\hat{\mathcal{A}}_{i}(\tau, \mathbf{z})=\hat{A}_{i}(0, \mathbf{z})-\int_{0}^{\tau} \hat{F}_{i \sigma}(\sigma, \mathbf{z}) \mathrm{d} \sigma .
$$

Remark 10. Assumption III, and consequently the validity of Theorem 9, is independent of the choice of the time axis for the definition of the foliation (20).

Proof of Theorem 9. (i) This potential obeys Theorem 23 in Appendix I. As $\mathcal{A}$ is of class $C^{2}$ on the Minkowski spacetime, so the assumption (29) of Theorem 6 and the assumption on continuous differentiability of the mapping $\tau \mapsto \hat{\mathcal{A}}_{i}(\tau, \mathbf{z})$ in Assumption II are clearly satisfied. Denote

$$
\xi(u)=u^{\kappa}, \quad \kappa<\varepsilon<\frac{1}{2} .
$$

The estimates of Theorem 23 imply then the following norm bounds:

$$
\begin{gathered}
\left\|\hat{F}_{i \tau}\right\|_{\infty},\left\|z^{i} \hat{F}_{i \tau}\right\|_{\infty} \leq \frac{\text { const }}{\langle\tau\rangle}, \\
\left\|\hat{F}_{i j}\right\|_{\infty},\left\|z^{i} \hat{F}_{i j}\right\|_{\infty} \leq \mathrm{const}, \\
\left\|\hat{\mathcal{A}}_{\tau}\right\|_{r, \tau} \leq \operatorname{const}\left(\frac{1+\log \langle\tau\rangle}{\langle\tau\rangle^{1+\varepsilon}}+\frac{\log \langle r\rangle}{\langle\tau\rangle^{3}}\right), \\
\left\|(1+\log \langle z\rangle)^{-1} \hat{\mathcal{A}}_{\tau}\right\|_{\infty}+\left\|\partial_{i} \hat{\mathcal{A}}_{\tau}\right\|_{\infty}+\left\|z^{i} \partial_{i} \hat{\mathcal{A}}_{\tau}\right\|_{\infty} \leq \operatorname{const} \frac{1+\log \langle\tau\rangle}{\langle\tau\rangle^{1+\varepsilon}}, \\
\left\|\partial_{i} \partial_{j} \hat{\mathcal{A}}_{\tau}\right\|_{\infty} \leq \operatorname{const}(1+\log \langle\tau\rangle), \\
\left\|\hat{\mathcal{A}}_{i}\right\|_{\infty}+\left\|z^{i} \hat{\mathcal{A}}_{i}\right\|_{\infty} \leq\left\|\xi(\langle z\rangle) \hat{\mathcal{A}}_{i}\right\|_{\infty}+\left\|\xi(\langle z\rangle) z^{i} \hat{\mathcal{A}}_{i}\right\|_{\infty} \leq \operatorname{const}(1+\log \langle\tau\rangle), \\
\left\|\partial_{\tau} \hat{\mathcal{A}}_{i}\right\|_{\infty}+\left\|z^{i} \partial_{\tau} \hat{\mathcal{A}}_{i}\right\|_{\infty} \leq \frac{\operatorname{const}}{\langle\tau\rangle}, \\
\left\|\partial_{i} \hat{\mathcal{A}}_{j}\right\|_{\infty}+\left\|z^{i} \partial_{i} \hat{\mathcal{A}}_{j}\right\|_{\infty}+\left\|z^{j} \partial_{i} \hat{\mathcal{A}}_{j}\right\|_{\infty}+\left\|z^{i} z^{j} \partial_{i} \hat{\mathcal{A}}_{j}\right\|_{\infty} \leq \operatorname{const}(1+\log \langle\tau\rangle) .
\end{gathered}
$$

It is now easily checked that the potential satisfies assumption (30) in Theorem 6 and all the remaining bounds in Assumption II, from which the thesis follows. (Note that in this case all expressions are bounded in $L^{\infty}$-norm, except for $\hat{\mathcal{A}}_{\tau}$.) 
(ii) Let the evolution operator $U(\tau, \sigma)$ refer to the potential defined in (i), and denote the new potential now considered by $\hat{\mathcal{A}}^{\mathcal{G}}$. Following further notation used in Theorem 6, we have

$$
\begin{aligned}
& \underset{\tau \rightarrow \pm \infty}{\mathrm{S}-\lim _{\tau}} U_{\Phi}^{\mathcal{G}}(\tau, 0)=\underset{\tau \pm \infty}{\mathrm{s}-\lim } \Phi^{*}(\tau) e^{i \mathcal{G}(\tau)} U(\tau, 0) e^{-i \mathcal{G}(0)}
\end{aligned}
$$

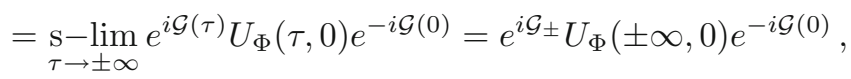

So

$$
\begin{gathered}
\underset{\tau \rightarrow \pm \infty}{\mathrm{s}-} \lim _{\tau} U_{0}(0, \tau) U^{\mathcal{G}}(\tau, 0)=U_{0 \Phi}(0, \pm \infty) U_{\Phi}^{\mathcal{G}}( \pm \infty, 0) \\
\quad=U_{0 \Phi}(0, \pm \infty) e^{i \mathcal{G}_{ \pm}} U_{\Phi}( \pm \infty, 0) \Omega_{\mp} e^{-i \mathcal{G}(0)} \equiv \Omega_{\mp}^{\mathcal{G}} .
\end{gathered}
$$

Similarly for the limits of the conjugated operators.

(iii) Both gauges are easily seen to satisfy condition (31) of Theorem 6; we turn to the estimates (32). In the case (a), with $C(x)=x \cdot A(x)$, we have

$$
\mathcal{G}(\tau, \mathbf{z})=-\log \langle z\rangle C(\tau, \mathbf{z}),
$$

and the estimates are easily checked with the use of the results of the proof of Theorem 23. Also, it follows from the estimate of $\left|\partial_{\tau} C\right|$ given there that $C(\tau, \mathbf{z})$ has limits for $\tau \rightarrow \pm \infty$.

In the case (b), it is now sufficient to investigate the difference of the gauge function $S_{\mathrm{tr}}(x)$ as compared to the gauge function of case (a):

$$
\mathcal{G}^{\prime}(x)=S_{\mathrm{tr}}(x)-S_{\log }(x) .
$$

Differentiating and using the form of $\partial_{\tau} x$ given in the proof of Theorem 23, we find

$$
\begin{aligned}
& \partial_{\tau} \mathcal{G}^{\prime}(\tau, \mathbf{z})=\langle\tau\rangle^{-2}\langle z\rangle A_{0}-\log \langle\tau\rangle \partial_{\tau} C \\
& \partial_{i} \partial_{\tau} \mathcal{G}^{\prime}(\tau, \mathbf{z})=\frac{1}{\langle\tau\rangle^{2}}\left[\frac{z^{i}}{\langle z\rangle} A_{0}+\langle z\rangle \partial_{i} A_{0}\right]-\log \langle\tau\rangle \partial_{i} \partial_{\tau} C,
\end{aligned}
$$

Now noting that $\partial_{i} \mathcal{G}(0, \mathbf{z})=0$, integrating (the last term by parts) and applying the derivative $\partial_{j}$, we obtain (fields in the integrand depend on $(\sigma, \mathbf{z})$ )

$$
\begin{aligned}
\partial_{i} \partial_{j} \mathcal{G}^{\prime}(\tau, \mathbf{z})= & \int_{0}^{\tau}\left[\frac{d^{i j}}{\langle z\rangle} A_{0}+\frac{1}{\langle z\rangle}\left(z^{i} \partial_{j} A_{0}+z^{j} \partial_{i} A_{0}\right)+\langle z\rangle \partial_{i} \partial_{j} A_{0}+\sigma \partial_{i} \partial_{j} C\right] \frac{\mathrm{d} \sigma}{\langle\sigma\rangle^{2}} \\
& -\log \langle\tau\rangle \partial_{i} \partial_{j} C,
\end{aligned}
$$

with $d^{i j}$ defined in (133). With the use of the estimates listed in the proof of Theorem 23, one finds

$$
\begin{array}{ll}
\left|\partial_{\tau} \mathcal{G}^{\prime}\right| \leq \operatorname{const} \frac{1+\log \langle\tau\rangle}{\langle\tau\rangle^{1+\varepsilon}}, \quad\left|\partial_{i} \partial_{\tau} \mathcal{G}^{\prime}\right| \leq \operatorname{const} \frac{1+\log \langle\tau\rangle}{\langle\tau\rangle^{1+\varepsilon}}, \\
\left|\partial_{i} \partial_{j} \mathcal{G}^{\prime}\right| \leq \operatorname{const}(1+\log \langle\tau\rangle), \quad\left|\partial_{i} \mathcal{G}^{\prime}\right| \leq \text { const }
\end{array}
$$

the fourth estimate by the integration of the second one. Thus, the estimates (32) are satisfied. Finally, $\partial_{\tau} \mathcal{G}^{\prime}$ is integrable on $\mathbb{R}$, so the thesis follows. 


\section{Discussion}

There are three questions we want to address in this section:

(i) How far is the present analysis from a complete treatment of the MaxwellDirac system?

(ii) Is there a further physical selection criterion to choose a gauge from the class of gauges obtained in Theorem 9?

(iii) Open problems.

With regard to the first of these questions, we note that the form of the charged currents producing electromagnetic fields in Assumption III mimics what one should expect in fully interacting theory. A possible shortage of this assumption rests in the estimates of derivatives of the currents, which would not be satisfied for oscillating terms in asymptotic behavior. The Dirac field current does have such asymptotic terms, but it is quite plausible to predict that oscillations dump the asymptotic behavior of fields produced by them. What would be needed is an appropriately fast vanishing of the leading oscillating asymptotic terms in the neighborhood of the lightcone (which is a quite reasonable prediction). This would presumably lead to similar behavior of electromagnetic potentials as that following from Assumption III. Moreover, we note that our Assumption II, on which our analysis is based, leaves much more room for the types of potentials than Assumption III.

Let us once more at this point recall the work by Flato et al. [7]. These authors do have a theorem on the evolution of the complete system, but in a rather restricted setting and with not much control over the range of validity. The theorem states that in the space of smooth (i.e. $C^{\infty}$ ) initial data there exists a neighborhood of zero, which gives rise to the Cauchy evolution and completeness. Our analysis is not fully developed to the full interacting case, but gives complete results for the Dirac part of the system, with the electromagnetic fields plausibly guessed.

Our main motivation for the present work was the expectation that the choice of gauge does matter for asymptotic behavior and its interpretation. We have shown that for a class of gauges the asymptotic behavior of the Dirac field approaches that of a free field, without the need for any dynamical corrections.

This brings us to the second question mentioned at the beginning. Theorem 9 identifies a class of gauges for which the situation described above takes place. The concrete gauge defined by (73) was used for technical reasons: this gauge satisfies Assumption II, and it is the only gauge with that property among the gauges explicitly mentioned in Theorem 9. However, we want to argue now, in less precise terms then those of the preceding sections, that a different choice of gauges in the given class has a definite physical interpretation when an extension, as mentioned above, to fully interacting system is considered. Such extension, partly based on conjectures, was considered in [8], and we have to briefly recall a few results of this analysis. It was found that if one uses inside the lightcone the gauge related to the Lorenz gauge $A$ (of 
similar properties as those of Assumption III) by

$$
\mathcal{A}_{\text {cone }}(x)=A(x)-\nabla S_{\text {cone }}(x), \quad S_{\text {cone }}(x)=\log \sqrt{x^{2}} x \cdot A(x),
$$

or another with similar timelike asymptotic behavior, then the asymptotic total four-momentum and angular momentum taken away into timelike infinity have the same functional form as those for the free Dirac field. Moreover, the energy-momentum radiated into null infinity is fully due to the free outgoing electromagnetic field. However, the angular momentum going out into the null infinity, in addition to the free radiated contribution, has mixed advout electromagnetic terms. These latter terms may be incorporated into the free Dirac field by a change of the asymptotic limit addition to the gauge function. As a result, both energy-momentum as well as angular momentum are clearly separated into electromagnetic and Dirac parts. ${ }^{11}$ Now we would like to identify in our class of global gauges those for which this separation may be expected. It is not difficult to show that for points inside the future lightcone, represented by $\lambda v$, with $\lambda>0$ and $v$ on the future unit hyperboloid, we have

$$
\lim _{\lambda \rightarrow \infty}\left[S_{\log }(\lambda v)-S_{\text {cone }}(\lambda v)\right]=0
$$

for the gauge function $S_{\log }$ defined in Theorem 9 (iii). Therefore, we put forward the selection criterion for the gauge functions $S$ to be used in (72) for the definition of $\mathcal{A}$ :

$$
S(\tau, \mathbf{z})=S_{\log }(\tau, \mathbf{z})+\Delta S(\tau, \mathbf{z}),
$$

where $\Delta S$ has the limit

$$
\Delta S(\infty, \mathbf{z})=\lim _{\tau \rightarrow \infty} \Delta S(\tau, \mathbf{z})
$$

which satisfies the condition of the separation of angular momentum as described above (it is easy to see that for $\Delta S$ sufficiently regular the rhs is equal to $\left.\lim _{\lambda \rightarrow \infty} \Delta S(\lambda(\langle z\rangle, \mathbf{z}))\right)$. Similar conditions should be applied for past infinity.

Gauges in the class thus selected have the property announced in the introduction: $x \cdot \mathcal{A}(x)$ vanishes asymptotically in timelike directions. This may be checked easily for $S_{\log }$, and if $\Delta S(\tau, \mathbf{z})$ is not oscillating in $\tau$, the same is true for this part.

The existence and completeness of the wave operators, as indicated in Remark 10, do not depend on the choice of the time axis for the definition of our spacetime foliation. On the other hand, and this is the first of open problems, the precise transformation law from one inertial observer to another needs further investigation. More generally, one can ask what is a general class of Cauchy foliations for which the results could be repeated, and how the results would depend on the choice in the class.

\footnotetext{
${ }^{11}$ For explicit expressions and more extensive discussion we refer the reader to [8], Section V. Here we would only like to reassure the reader that the problems met for angular momentum in electrodynamics are accounted for in that discussion.
} 
Let us end this discussion by expressing the belief that our scheme could be applied to analogous problems on at least some of the smooth curved spacetime backgrounds. Whether, as inquired by one of the Referees, further extension to black hole type spacetimes would be possible, is a more speculative question.

Open Access. This article is licensed under a Creative Commons Attribution 4.0 International License, which permits use, sharing, adaptation, distribution and reproduction in any medium or format, as long as you give appropriate credit to the original author(s) and the source, provide a link to the Creative Commons licence, and indicate if changes were made. The images or other third party material in this article are included in the article's Creative Commons licence, unless indicated otherwise in a credit line to the material. If material is not included in the article's Creative Commons licence and your intended use is not permitted by statutory regulation or exceeds the permitted use, you will need to obtain permission directly from the copyright holder. To view a copy of this licence, visit http://creativecommons.org/ licenses/by/4.0/.

Publisher's Note Springer Nature remains neutral with regard to jurisdictional claims in published maps and institutional affiliations.

\section{Appendix A. Transformation $K$}

The operator of the Lorentz rotation of Dirac spinors in the hyperplane spanned by the timelike, future-pointing unit vectors $t$ and $n$ is given by

$$
K(\kappa)=\exp \left(\frac{\kappa}{2}[\gamma \cdot n, \gamma \cdot t]\right)=\cosh (\kappa \sinh \zeta)+\frac{\sinh (\kappa \sinh \zeta)}{2 \sinh \zeta}[\gamma \cdot n, \gamma \cdot t],
$$

where $\kappa$ is the parameter of the rotation, $\zeta>0$ is such that $n \cdot t=\cosh \zeta$, and the rhs is obtained by a simple calculation with the use of the relation $([\gamma \cdot n, \gamma \cdot t])^{2}=4(\sinh \zeta)^{2}$. Rotation of $\gamma \cdot n$ gives

$$
K(-\kappa) \gamma \cdot n K(\kappa)=\frac{\sinh (\zeta-2 \kappa \sinh \zeta) \gamma \cdot n+\sinh (2 \kappa \sinh \zeta) \gamma \cdot t}{\sinh \zeta} .
$$

Demanding that the coefficient at $\gamma \cdot n$ vanishes, we find $\kappa_{0}=\zeta /(2 \sinh \zeta)$ and then for $K=K\left(\kappa_{0}\right)$ we have

$$
\begin{aligned}
& K=\frac{1+\gamma \cdot n \gamma \cdot t}{\sqrt{2(1+n \cdot t)}}=\gamma \cdot w \beta=K^{\dagger}, \quad w=\frac{t+n}{\sqrt{(t+n)^{2}}}, \\
& K^{-1} \gamma \cdot n K=\gamma \cdot t .
\end{aligned}
$$

We now put $t=e_{0}$, and $n$ as given in (7). With notation introduced in Sect. 2, we now have:

Proposition 11. If $\Sigma_{\tau}$ are rotationally symmetric, that is $\tau=\tau\left(x^{0},|\mathbf{x}|\right)$, then

$$
\left[\hat{\gamma}_{K}^{\mu},\left(K^{-1} \partial_{\mu} K\right)\right]_{+}=0
$$


Proof. The lhs of (75) is coordinate independent, so we can use Minkowski coordinates. If we use $K$ in the form (74), then we find

$$
\beta\left[\gamma_{K}^{a},\left(K^{-1} \nabla_{a} K\right)\right]_{+} \beta=\left(\gamma^{b} \gamma^{a} \gamma^{c}-\gamma^{c} \gamma^{a} \gamma^{b}\right) w_{b} \nabla_{a} w_{c}=2 \gamma^{b} \gamma^{a} \gamma^{c} w_{[b} \nabla_{a} w_{c]},
$$

where the second equality follows from the easily seen complete antisymmetry of the expression in gamma matrices following the first equality sign. Using the form of $w$ given in (74), we find

$$
w_{[b} \nabla_{a} w_{c]}=\left[(t+n)^{2}\right]^{-1}\left(t_{[b}+n_{[b}\right) \nabla_{a} n_{c]} .
$$

If the assumption of the proposition holds, then $n_{a}=\left(k_{1}, k_{2} x^{i}\right)$, where $k_{1}, k_{2}$ are functions of $x^{0}$ and $|\mathbf{x}|$. In consequence, the rhs of (76) vanishes and the thesis follows.

\section{Appendix B. An Open System}

We discuss here a class of open systems for which the (general) Schrödinger equation may be defined. The main tool for the discussion of this section is the commutator theorem, as formulated in Thm. X.37 in [15]. ${ }^{12}$

\section{B.1: Hamiltonians}

Theorem 12. Let $h_{0} \geq 1$ be a self-adjoint operator, for which the dense subspace $\mathcal{D} \subseteq \mathcal{D}\left(h_{0}\right)$ is a core. Suppose that for $\tau \in \mathbb{R}$ the following holds:

(a) $h(\tau)$ is a family of symmetric operators on $\mathcal{D}$,

(b) the commutator $\left[h(\tau), h_{0}\right]$, defined originally as a quadratic form on $\mathcal{D}$, extends to an operator on $\mathcal{D}$,

(c) for $\psi \in \mathcal{D}$ the maps $\tau \mapsto h(\tau) \psi, \tau \mapsto\left[h(\tau), h_{0}\right] \psi$ are strongly continuous and the following bounds are satisfied:

$$
\begin{aligned}
\|h(\tau) \psi\| & \leq c_{1}(\tau)\left\|h_{0} \psi\right\|, \\
\left|\left(\psi,\left[h(\tau), h_{0}\right] \psi\right)\right| & \leq c_{2}(\tau)\left(\psi, h_{0} \psi\right), \\
\left\|\left[h(\tau), h_{0}\right] \psi\right\| & \leq c_{3}(\tau)\left\|h_{0} \psi\right\|,
\end{aligned}
$$

where $c_{i}(\tau)$ are continuous functions.

Denote by the same symbols the closures of the operators $h(\tau)$ and $\left[h(\tau), h_{0}\right]$. Then the following holds:

(A) $\mathcal{D}\left(h_{0}\right) \subseteq \mathcal{D}(h(\tau)), \mathcal{D}\left(h_{0}\right) \subseteq \mathcal{D}\left(\left[h(\tau), h_{0}\right]\right)$, and $h(\tau)$ are essentially selfadjoint on $\mathcal{D}$ and on each core of $h_{0}$,

(B) operators $h(\tau) h_{0}^{-1}, h_{0}^{-1} h(\tau),\left[h(\tau), h_{0}\right] h_{0}^{-1}$ and $h_{0}^{-1}\left[h(\tau), h_{0}\right]$ extend to bounded operators, strongly continuous with respect to $\tau$, and

$$
\begin{aligned}
& \left\|h(\tau) h_{0}^{-1}\right\|=\left\|h_{0}^{-1} h(\tau)\right\| \leq c_{1}(\tau), \\
& \left\|\left[h(\tau), h_{0}\right] h_{0}^{-1}\right\|=\left\|h_{0}^{-1}\left[h(\tau), h_{0}\right]\right\| \leq c_{3}(\tau),
\end{aligned}
$$

(C) statement (c) remains true for all $\psi \in \mathcal{D}\left(h_{0}\right)$.

\footnotetext{
${ }^{12}$ Unlike in [11], here we make no use of the Kato theorem.
} 
Proof. As $\mathcal{D}$ is a core for $h_{0}$, the domains inclusions in (A) and the extension of inequalities $(77)-(79)$ to $\psi \in \mathcal{D}\left(h_{0}\right)$ follow immediately, if we denote the closures of the operators $h(\tau)$ and $\left[h(\tau), h_{0}\right]$ by the same symbols. In addition, the essential self-adjointness of $h(\tau)$ follows from (77) and (78) by the commutator theorem, Thm. X.37 in [15].

The bounds (80) for the operators $h(\tau) h_{0}^{-1}$ and $\left[h(\tau), h_{0}\right] h_{0}^{-1}$ follow now immediately. The operators $h_{0}^{-1} h(\tau)$ and $h_{0}^{-1} i\left[h(\tau), h_{0}\right]$, initially densely defined on $\mathcal{D}\left(h_{0}\right)$, extend to conjugates of $h(\tau) h_{0}^{-1}$ and $i\left[h(\tau), h_{0}\right] h_{0}^{-1}$, respectively, so all the bounds (80) are satisfied.

Strong continuity in $\tau$ of the operators $h_{0}^{-1} h(\tau)$ and $h_{0}^{-1}\left[h(\tau), h_{0}\right]$ follows immediately from the bounds (80) and the assumption (c). For the continuity of $h(\tau) h_{0}^{-1}$, we note what follows. For $\psi \in \mathcal{H}$ the vector $h_{0}^{-1} \psi$ is in $\mathcal{D}\left(h_{0}\right)$, so for each $\epsilon>0$ there exist $\varphi \in \mathcal{D}$ (a core for $h_{0}$ ) such that $\left\|\psi-h_{0} \varphi\right\| \leq \epsilon$. We estimate

$$
\left\|h(\tau) h_{0}^{-1} \psi-h(\sigma) h_{0}^{-1} \psi\right\| \leq \epsilon\left[c_{1}(\tau)+c_{1}(\sigma)\right]+\|h(\tau) \varphi-h(\sigma) \varphi\|,
$$

so the strong continuity follows. The case of $\left[h(\tau), h_{0}\right] h_{0}^{-1}$ is analogous. This completes the proof of $(\mathrm{B})$, and its consequence is the statement of strong continuity of maps in (C).

\section{B.2: Evolution}

Theorem 13. Let all the assumptions of Theorem 12 be satisfied. Moreover, let $(\tau, \sigma) \mapsto u(\tau, \sigma)$ be a strongly continuous unitary evolution system in $\mathcal{H}$ :

$$
u(\tau, \rho) u(\rho, \sigma)=u(\tau, \sigma), \quad u(\tau, \tau)=1,
$$

such that $u(\tau, \sigma) \mathcal{D}=\mathcal{D}$. Suppose that for $\psi \in \mathcal{D}$ :

(a) the maps $(\tau, \sigma) \mapsto h(\tau) u(\tau, \sigma) \psi$ and $(\tau, \sigma) \mapsto h_{0} u(\tau, \sigma) \psi$ are strongly continuous,

(b) the $\operatorname{map}(\tau, \sigma) \mapsto u(\tau, \sigma) \psi$ is of class $C^{1}$ in the strong sense and the following equations hold:

$$
i \partial_{\tau} u(\tau, \sigma) \psi=h(\tau) u(\tau, \sigma) \psi, \quad i \partial_{\sigma} u(\tau, \sigma) \psi=-u(\tau, \sigma) h(\sigma) \psi,
$$

Then, the following holds:

(A) the operator $h_{0} u(\tau, \sigma) h_{0}^{-1}$ is bounded, with the norm

$$
\left\|h_{0} u(\tau, \sigma) h_{0}^{-1}\right\| \leq \exp \left[\operatorname{const} \int_{\sigma}^{\tau} c_{3}(\rho) \mathrm{d} \rho\right],
$$

so in particular

$$
u(\tau, \sigma) \mathcal{D}\left(h_{0}\right)=\mathcal{D}\left(h_{0}\right) ;
$$

(B) the $\operatorname{map}(\tau, \sigma) \mapsto h_{0} u(\tau, \sigma) h_{0}^{-1}$ is strongly continuous;

(C) all statements of assumptions (a) and (b) remain true for all $\psi \in \mathcal{D}\left(h_{0}\right)$.

Proof. To show (A), we use a standard trick. ${ }^{13}$ It is easy to see that

$$
\left[\frac{h_{0}^{2}}{1+\epsilon h_{0}^{2}}, h(\tau)\right]=\frac{h_{0}}{1+\epsilon h_{0}^{2}}\left[\left[h(\tau), h_{0}\right], h_{0}^{-1}\right]+\frac{h_{0}}{1+\epsilon h_{0}^{2}},
$$

\footnotetext{
${ }^{13}$ See, e.g., Proposition B.3.3 in [2].
} 
so using the second bound in (80), we have for $\psi \in \mathcal{D}$

$$
\partial_{\tau}\left(u(\tau, \sigma) \psi, \frac{h_{0}^{2}}{1+\epsilon h_{0}^{2}} u(\tau, \sigma) \psi\right) \leq 2 c_{3}(\tau)\left(u(\tau, \sigma) \psi, \frac{h_{0}^{2}}{1+\epsilon h_{0}^{2}} u(\tau, \sigma) \psi\right),
$$

By Gronwall's inequality, we obtain

$$
\left\|\frac{h_{0}}{\left(1+\epsilon h_{0}^{2}\right)^{\frac{1}{2}}} u(\tau, \sigma) \psi\right\| \leq e^{\int_{\sigma}^{\tau} c_{3}(\rho) \mathrm{d} \rho}\left\|\frac{h_{0}}{\left(1+\epsilon h_{0}^{2}\right)^{\frac{1}{2}}} \psi\right\| .
$$

As $h_{0}$ is closed, letting $\epsilon \searrow 0$ we show that $u(\tau, \sigma) \psi \in \mathcal{D}\left(h_{0}\right)$ for $\psi \in \mathcal{D}$, and

$$
\left\|h_{0} u(\tau, \sigma) \psi\right\| \leq e^{\int_{\sigma}^{\tau} c_{3}(\rho) \mathrm{d} \rho}\left\|h_{0} \psi\right\| .
$$

But $\mathcal{D}$ is a core for $h_{0}$, so this inequality extends to $\mathcal{D}\left(h_{0}\right)$. Setting $\psi=h_{0}^{-1} \chi$, with arbitrary $\chi \in \mathcal{H}$, we obtain (A).

For statement (B) we note that for $\psi \in \mathcal{H}$ and $\varphi \in \mathcal{D}$ such that $\left\|\psi-h_{0} \varphi\right\| \leq \epsilon($ as in the proof of Theorem 12) we have

$$
\begin{aligned}
& \left\|h_{0}\left[u(\tau, \sigma)-u\left(\tau^{\prime}, \sigma^{\prime}\right)\right] h_{0}^{-1} \psi\right\| \\
& \quad \leq\left\|h_{0}\left[u(\tau, \sigma)-u\left(\tau^{\prime}, \sigma^{\prime}\right)\right] h_{0}^{-1}\right\| \epsilon+\left\|h_{0} u(\tau, \sigma) \varphi-h_{0} u\left(\tau^{\prime}, \sigma^{\prime}\right) \varphi\right\|,
\end{aligned}
$$

so the map $(\tau, \sigma) \mapsto h_{0} u(\tau, \sigma) h_{0}^{-1}$ is strongly continuous.

The extension of (a) to all $\psi \in \mathcal{D}\left(h_{0}\right)$ now follows from the present statement (B), and the statement (B) of Theorem 12. For the extension of (b), we write the integral forms of equations $(81)$ for $\psi \in \mathcal{D}$

$$
u(\tau, \sigma) \psi-\psi=-i \int_{\sigma}^{\tau} h(\rho) u(\rho, \sigma) \psi \mathrm{d} \rho=-i \int_{\sigma}^{\tau} u(\rho, \sigma) h(\rho) \psi \mathrm{d} \rho .
$$

But we already know that both integrands extend to continuous functions of $\rho$ for $\psi \in \mathcal{D}\left(h_{0}\right)$, so this extension followed by differentiation leads to the extended equations (81).

\section{Appendix C. A Lemma}

Here we state a result to be applied in Sects. 2 and 3. The lemma below enables the application of Theorem 12 to our system.

Lemma 14. Let $L^{i}(\mathbf{z}), i=1,2,3$, and $M(\mathbf{z})$ be four $n \times n$ Hermitian matrix functions on $\mathbb{R}^{3}$, and denote

$$
h=\frac{1}{2}\left(L^{i} p_{i}+p_{i} L^{i}\right)+M, \quad h_{0}=\frac{1}{2}\left(\mathbf{p}^{2}+\mathbf{z}^{2}\right) .
$$

Then for some constants $C_{i}(i=1,2,3)$ and all $\varphi \in C_{0}^{\infty}\left(\mathbb{R}^{3}, \mathbb{C}^{n}\right)$ the following inequalities are satisfied:

(i) if the functions $\langle z\rangle^{-1} L^{i}(\mathbf{z}), \partial_{i} L^{j}(\mathbf{z})$ and $\langle z\rangle^{-2} M(\mathbf{z})$ are in $L^{\infty}\left(\mathbb{R}^{3}\right)$, then

$$
\|h \varphi\| \leq C_{1}\left\|h_{0} \varphi\right\|
$$

(ii) if in addition $\partial_{i} \partial_{j} L^{k}(\mathbf{z})$ and $\langle z\rangle^{-1} \partial_{i} M(\mathbf{z})$ are in $L^{\infty}\left(\mathbb{R}^{3}\right)$, then

$$
\left|\left(h \psi, h_{0} \varphi\right)-\left(h_{0} \psi, h \varphi\right)\right| \leq C_{2}\left\|h_{0}^{\frac{1}{2}} \psi\right\|\left\|h_{0}^{\frac{1}{2}} \varphi\right\| ;
$$


(iii) if in addition to (i) and (ii) also $\partial_{i} \partial_{j} \partial_{k} L^{l}(\mathbf{z})$ and $\partial_{i} \partial_{j} M(\mathbf{z})$ are in $L^{\infty}\left(\mathbb{R}^{3}\right)$, then

$$
\left\|\left[h, h_{0}\right] \varphi\right\| \leq C_{3}\left\|h_{0} \varphi\right\| .
$$

Proof. ${ }^{14}$ Before starting the proof of (i)-(iii), we note a few simple facts. For $\varphi \in C_{0}^{\infty}\left(\mathbb{R}^{3}, \mathbb{C}^{n}\right)$ we note the identity

$$
\varphi^{\dagger} \mathbf{p}^{2} \varphi+\left(\mathbf{p}^{2} \varphi\right)^{\dagger} \varphi+\Delta|\varphi|^{2}=2|\mathbf{p} \varphi|^{2},
$$

which multiplied by $\mathbf{z}^{2}$ and integrated (transfer $\Delta$ by parts) gives

$$
\operatorname{Re}\left(\mathbf{p}^{2} \varphi, \mathbf{z}^{2} \varphi\right)+3\|\varphi\|^{2}=\|\mathbf{z} \mid \mathbf{p} \varphi\|^{2} .
$$

Also, it follows from $\mathbf{p}^{2}+\mathbf{z}^{2} \geq 3 \cdot \mathbf{1}$ that

$$
\|\varphi\| \leq \frac{1}{3}\left\|\left(\mathbf{p}^{2}+\mathbf{z}^{2}\right) \varphi\right\| .
$$

Using (85) and (86), one finds

$$
\begin{aligned}
& \left\|\left(\mathbf{p}^{2}+\mathbf{z}^{2}\right) \varphi\right\|^{2}-\left\|\mathbf{p}^{2} \varphi\right\|^{2}-\left\|\mathbf{z}^{2} \varphi\right\|^{2}=2 \operatorname{Re}\left(\mathbf{p}^{2} \varphi, \mathbf{z}^{2} \varphi\right) \\
& \quad \geq-6\|\varphi\|^{2} \geq-\frac{2}{3}\left\|\left(\mathbf{p}^{2}+\mathbf{z}^{2}\right) \varphi\right\|^{2},
\end{aligned}
$$

which gives the bound:

$$
\left\|\mathbf{p}^{2} \varphi\right\|^{2}+\left\|\mathbf{z}^{2} \varphi\right\|^{2} \leq \frac{5}{3}\left\|\left(\mathbf{p}^{2}+\mathbf{z}^{2}\right) \varphi\right\|^{2} .
$$

We note that the $L$ - and $M$-parts of the thesis may be proved separately. Consider the $L$-part first, and assume that $L^{i}$ satisfy the assumptions in (i). Then $N_{\alpha, i \beta}(\mathbf{z})=\langle z\rangle^{-1} L_{\alpha \beta}^{i}(\mathbf{z})$ may be treated as a bounded operator $\mathbb{C}^{3 n} \otimes L^{2}\left(\mathbb{R}^{3}\right) \mapsto \mathbb{C}^{n} \otimes L^{2}\left(\mathbb{R}^{3}\right)$, whose norm we denote $d_{1}$. Then,

$$
\begin{aligned}
\left\|L^{i} p_{i} \varphi\right\|^{2} & \leq d_{1}^{2} \sum_{i}\left\|\langle z\rangle p_{i} \varphi\right\|^{2}=d_{1}^{2}\left(\||\mathbf{z}| \mathbf{p} \varphi\|^{2}+\left(\varphi, \mathbf{p}^{2} \varphi\right)\right) \\
& \leq d_{1}^{2}\left(\left\|\mathbf{p}^{2} \varphi\right\|\left\|\mathbf{z}^{2} \varphi\right\|+3\|\varphi\|^{2}+\|\varphi\|\left\|\mathbf{p}^{2} \varphi\right\|\right) \\
& \leq \frac{1}{2} d_{1}^{2}\left(2\left\|\mathbf{p}^{2} \varphi\right\|^{2}+\left\|\mathbf{z}^{2} \varphi\right\|^{2}+7\|\varphi\|^{2}\right)
\end{aligned}
$$

where in the third step we used identity (85) and the Schwarz inequality. Taking into account that $\mathbf{L} \cdot \mathbf{p}+\mathbf{p} \cdot \mathbf{L}=2 \mathbf{L} \cdot \mathbf{p}-i(\boldsymbol{\partial} \cdot \mathbf{L})$ and $\|\boldsymbol{\partial} \cdot \mathbf{L}\|_{\infty} \leq$ const, with the use of (86) and (87) one easily arrives at the estimate (82) for the $L$-part.

Let now $L^{i}$ satisfy the assumptions in (ii) and let $\varphi \in C_{0}^{\infty}\left(\mathbb{R}^{3}, \mathbb{C}^{n}\right)$. Then, using the usual rules for calculation of commutators, one finds

$$
\left[\left[L^{i}, p_{i}\right]_{+}, h_{0}\right] \varphi=-2 i z^{i} L^{i} \varphi+\frac{i}{2}\left[p_{i}, p_{j}\left(\partial_{j} L^{i}\right)+\left(\partial_{j} L^{i}\right) p_{j}\right]_{+} \varphi,
$$

which makes sense as a distributional identity $\left(p_{i}, p_{j}\right.$ treated distributionally). As $L^{i}$ are twice differentiable, we can transform the second term on the rhs:

$$
\frac{i}{2}\left[p_{i}, p_{j}\left(\partial_{j} L^{i}\right)+\left(\partial_{j} L^{i}\right) p_{j}\right]_{+} \varphi=i p_{i}\left(\left(\partial_{j} L^{i}\right)+\left(\partial_{i} L^{j}\right)\right) p_{j} \varphi+\frac{i}{2}\left[p_{i}, \Delta L^{i}\right] \varphi .
$$

\footnotetext{
${ }^{14}$ It may be remarked that the proof admits some weakening of the assumptions. For instance, in (i) for the derivatives of $L^{i}$ it would be sufficient that $\langle z\rangle^{-2} \partial_{i} L^{i}$ be in $L^{\infty}$; in (ii) for the second derivatives of $L^{i}$ it suffices that $\langle z\rangle \Delta L^{i}$ is in $L^{\infty}$. We shall not need such more general results.
} 
Setting this into (88) and taking the product with $\psi \in C_{0}^{\infty}\left(\mathbb{R}^{3}, \mathbb{C}^{n}\right)$, we obtain

$$
\begin{aligned}
& \left(\left[L^{i}, p_{i}\right]_{+} \psi, h_{0} \varphi\right)-\left(h_{0} \psi,\left[L^{i}, p_{i}\right]_{+} \varphi\right)=i\left(p_{i} \psi,\left(\partial_{i} L^{j}+\partial_{j} L^{i}\right) p_{j} \varphi\right) \\
& -\frac{1}{2}\left(\psi,\left(\Delta L^{i}\right) p_{i} \varphi\right)+\frac{1}{2}\left(\left(\Delta L^{i}\right) p_{i} \psi, \varphi\right)-2 i\left(z^{i} \psi, L^{i} \varphi\right) .
\end{aligned}
$$

We treat $P_{i \alpha, j \beta}(\mathbf{z})=\partial_{i} L_{\alpha \beta}^{j}(\mathbf{z})+\partial_{j} L_{\alpha \beta}^{i}(\mathbf{z})$ as a bounded operator acting in $\mathbb{C}^{3 n} \otimes L^{2}\left(\mathbb{R}^{3}\right)$, whose norm we denote $d_{2}$. Then

$$
\left|\left(p_{i} \psi,\left(\partial_{i} L^{j}+\partial_{j} L^{i}\right) p_{j} \varphi\right)\right| \leq d_{2}\left(\sum_{i}\left\|p_{i} \psi\right\|^{2} \sum_{j}\left\|p_{j} \varphi\right\|^{2}\right)^{\frac{1}{2}} \leq 2 d_{2}\left\|h_{0}^{\frac{1}{2}} \psi\right\|\left\|h_{0}^{\frac{1}{2}} \varphi\right\| .
$$

Similarly, $Q_{\alpha, i \beta}(\mathbf{z})=\Delta L_{\alpha \beta}^{i}(\mathbf{z})$ is a bounded operator from $\mathbb{C}^{3 n} \otimes L^{2}\left(\mathbb{R}^{3}\right)$ into $\mathbb{C}^{n} \otimes L^{2}\left(\mathbb{R}^{3}\right)$ with the norm $d_{3}$, and $R_{i \alpha, \beta}(\mathbf{z})=\langle z\rangle^{-1} L_{\alpha \beta}^{i}(\mathbf{z})$ a bounded operator from $\mathbb{C}^{n} \otimes L^{2}\left(\mathbb{R}^{3}\right)$ into $\mathbb{C}^{3 n} \otimes L^{2}\left(\mathbb{R}^{3}\right)$ with the norm $d_{4}$. Therefore

$$
\begin{aligned}
& \left|\left(\psi,\left(\Delta L^{i}\right) p_{i} \varphi\right)\right| \leq d_{3}\|\psi\|\left(\sum_{i}\left\|p_{i} \varphi\right\|^{2}\right)^{\frac{1}{2}} \leq \mathrm{const}\left\|h_{0}^{\frac{1}{2}} \psi\right\|\left\|h_{0}^{\frac{1}{2}} \varphi\right\|, \\
& \left|\left(z^{i} \psi, L^{i} \varphi\right)\right| \leq d_{4}\|\langle z\rangle \varphi\|\left(\sum_{i}\left\|z^{i} \psi\right\|^{2}\right)^{\frac{1}{2}} \leq \mathrm{const}\left\|h_{0}^{\frac{1}{2}} \psi\right\|\left\|h_{0}^{\frac{1}{2}} \varphi\right\|,
\end{aligned}
$$

which ends the proof of (83) for the $L$-part.

If in addition the assumptions in (iii) are satisfied, then on the rhs of (88) all operators $p_{i}$ may be commuted to the right, and the commutator is a differential operator with functional coefficients:

$$
\left[\left[L^{i}, p_{i}\right]_{+}, h_{0}\right]=2 i\left(\partial_{i} L^{j}\right) p_{i} p_{j}+\left(\Delta L^{j}+\partial_{j} \boldsymbol{\partial} \cdot \mathbf{L}\right) p_{j}-\frac{i}{2}(\Delta \boldsymbol{\partial} \cdot \mathbf{L})-2 i \mathbf{z} \cdot \mathbf{L} .
$$

Using similar techniques as above, we find

$$
\begin{aligned}
& \left\|\left(\partial_{i} L^{j}\right) p_{i} p_{j} \varphi\right\| \leq d_{5}\left(\sum_{i, j}\left\|p_{i} p_{j} \varphi\right\|^{2}\right)^{\frac{1}{2}}=d_{5}\left\|\mathbf{p}^{2} \varphi\right\|, \\
& \left\|\left(\Delta L^{j}+\partial_{j} \boldsymbol{\partial} \cdot \mathbf{L}\right) p_{j} \varphi\right\| \leq d_{6}\left(\sum_{j}\left\|p_{j} \varphi\right\|^{2}\right)^{\frac{1}{2}} \leq \mathrm{const}\left\|h_{0}^{\frac{1}{2}} \varphi\right\| \leq \mathrm{const}\left\|h_{0} \varphi\right\|, \\
& \|(\Delta \boldsymbol{\partial} \cdot \mathbf{L}) \varphi\| \leq \mathrm{const}\|\varphi\|, \quad\|\mathbf{z} \cdot \mathbf{L} \varphi\| \leq \mathrm{const}\left\|\langle z\rangle^{2} \varphi\right\| .
\end{aligned}
$$

Again using (86) and (87), one obtains (84) for the $L$-part.

For the $M$-part, note that

$$
\|M \varphi\| \leq \mathrm{const}\left\|\langle z\rangle^{2} \varphi\right\| \leq \mathrm{const}\left\|\left(\mathbf{p}^{2}+\mathbf{z}^{2}\right) \varphi\right\|,
$$

which proves (82). Consider the commutator

$$
\left[M, h_{0}\right]=\frac{i}{2}[(\boldsymbol{\partial} M) \cdot \mathbf{p}+\mathbf{p} \cdot(\boldsymbol{\partial} M)] .
$$

If the assumptions in (ii) are satisfied, then

$$
\begin{aligned}
& 2\left|\left(M \psi, h_{0} \varphi\right)-\left(h_{0} \psi, M \varphi\right)\right| \leq\left|\left(\left(\partial_{i} M\right) \psi, p_{i} \varphi\right)\right|+\left|\left(p_{i} \psi,\left(\partial_{i} M\right) \varphi\right)\right| \\
& \quad \leq \mathrm{const}\left\|h_{0}^{\frac{1}{2}} \psi\right\|\left\|h_{0}^{\frac{1}{2}} \varphi\right\|,
\end{aligned}
$$

which proves (83). Finally, with the assumption in (iii), (84) for this part is obtained similarly as (82) for the $L$-part. 


\section{Appendix D. Special Variables}

Variables $(\tau, \mathbf{z})$ are defined by:

$$
x^{0}=\tau\langle z\rangle, \quad \mathbf{x}=\langle\tau\rangle \mathbf{z},
$$

see (20). Further notation:

$$
\langle\tau \mid z\rangle=\left(\tau^{2}+\mathbf{z}^{2}+1\right)^{\frac{1}{2}}, \quad\langle\tau \| z\rangle=\langle\tau\rangle\langle z\rangle+\langle\tau \mid z\rangle .
$$

At various points of the calculations in this section, the following identities are used

$$
\tau^{2}|\mathbf{z}|^{2}=\langle\tau\rangle^{2}\langle z\rangle^{2}-\langle\tau \mid z\rangle^{2}=\langle\tau \| z\rangle(\langle\tau\rangle\langle z\rangle-\langle\tau \mid z\rangle) .
$$

The derivative transformation between these coordinates and the Minkowski system is given by

$$
\frac{\partial\left(x^{0}, \mathbf{x}\right)}{\partial(\tau, \mathbf{z})}=\left(\begin{array}{cc}
\langle z\rangle & \frac{\tau \mathbf{z}^{\boldsymbol{\top}}}{\langle z\rangle} \\
\frac{\tau \mathbf{z}}{\langle\tau\rangle} & \langle\tau\rangle \mathbf{1}
\end{array}\right), \quad \frac{\partial(\tau, \mathbf{z})}{\partial\left(x^{0}, \mathbf{x}\right)}=\left(\begin{array}{cc}
\frac{\langle\tau\rangle^{2}\langle z\rangle}{\langle\tau \mid z\rangle^{2}} & -\frac{\tau\langle\tau\rangle \mathbf{z}^{\top}}{\langle\tau \mid z\rangle^{2}} \\
-\frac{\tau\langle z\rangle \mathbf{z}}{\langle\tau \mid z\rangle^{2}} & \frac{1}{\langle\tau\rangle}\left[\mathbf{1}+\frac{\tau^{2}}{\langle\tau \mid z\rangle^{2}} \mathbf{z z}^{\boldsymbol{\top}}\right]
\end{array}\right] .
$$

Using these transformations, one finds the new metric tensor matrices

$$
\begin{aligned}
& \left(g_{\mu \nu}\right)=\left(\begin{array}{cc}
\frac{\langle\tau \mid z\rangle^{2}}{\langle\tau\rangle^{2}} & 0 \\
0 & -\langle\tau\rangle^{2} \mathbf{1}+\frac{\tau^{2}}{\langle z\rangle^{2}} \mathbf{z z}^{\top}
\end{array}\right), \quad g_{(z)}=-\frac{\langle\tau\rangle^{4}\langle\tau \mid z\rangle^{2}}{\langle z\rangle^{2}} \\
& \left(g^{\mu \nu}\right)=\left(\begin{array}{cc}
\frac{\langle\tau\rangle^{2}}{\langle\tau \mid z\rangle^{2}} & 0 \\
0 & -\frac{1}{\langle\tau\rangle^{2}}\left[\mathbf{1}+\frac{\tau^{2}}{\langle\tau \mid z\rangle^{2}} \mathbf{z z}^{\top}\right]
\end{array}\right)
\end{aligned}
$$

and the new vector components of the gamma matrices

$$
\begin{aligned}
& \hat{\gamma}^{\tau}=\frac{\langle\tau\rangle}{\langle\tau \mid z\rangle^{2}}\left(\langle\tau\rangle\langle z\rangle \gamma^{0}-\tau \mathbf{z} \cdot \gamma\right), \\
& \hat{\gamma}^{i}=\frac{1}{\langle\tau\rangle} \gamma^{i}-\frac{\tau}{\langle\tau\rangle\langle\tau \mid z\rangle^{2}}\left(\langle\tau\rangle\langle z\rangle \gamma^{0}-\tau \mathbf{z} \cdot \gamma\right) z^{i} .
\end{aligned}
$$

The operator $K$ defined by (8) and discussed in Appendix A takes the form

$$
\begin{aligned}
& K=\frac{1}{\sqrt{2\langle\tau \mid z\rangle}}\left[\sqrt{\langle\tau \| z\rangle}+\frac{\tau}{\sqrt{\langle\tau \| z\rangle}} \gamma^{0} \mathbf{z} \cdot \gamma\right]=K^{\dagger}, \\
& K^{-1}=\gamma^{0} K \gamma^{0}
\end{aligned}
$$

which leads to the following form of the transformed gamma matrices (10) and $\lambda$ matrices introduced by (11): 


$$
\begin{aligned}
\hat{\gamma}_{K}^{i} & =\frac{1}{\langle\tau\rangle} \gamma_{\perp}^{i}+\frac{\langle z\rangle}{\langle\tau \mid z\rangle} \gamma_{z} \hat{z}^{i} \\
\lambda^{i} & =\frac{\langle\tau \mid z\rangle}{\langle\tau\rangle^{2}} \alpha_{\perp}^{i}+\frac{\langle z\rangle}{\langle\tau\rangle} \alpha_{z} \hat{z}^{i} \\
& =\frac{\langle\tau \mid z\rangle}{\langle\tau\rangle^{2}} \alpha_{\perp}^{i}+\frac{1}{(\langle z\rangle+|\mathbf{z}|)\langle\tau\rangle} \alpha_{z} \hat{z}^{i}+\frac{1}{\langle\tau\rangle} \alpha_{z} z^{i}
\end{aligned}
$$

where

$$
\begin{array}{lll}
\hat{z}^{i}=\frac{z^{i}}{|\mathbf{z}|}, & \gamma_{z}=\hat{\mathbf{z}} \cdot \boldsymbol{\gamma}, & \gamma_{\perp}^{i}=\gamma^{i}-\gamma_{z} \hat{z}^{i}, \\
\alpha^{i}=\gamma^{0} \gamma^{i}, & \alpha_{z}=\hat{\mathbf{z}} \cdot \boldsymbol{\alpha}, & \alpha_{\perp}^{i}=\alpha^{i}-\alpha_{z} \hat{z}^{i} .
\end{array}
$$

For the estimation of $X$ in (58) it is convenient to have

$$
\left(g^{\tau \tau}\right)^{\frac{1}{2}} \lambda^{i}=\frac{1}{\langle\tau\rangle} \alpha_{\perp}^{i}+\frac{1}{(\langle z\rangle+|\mathbf{z}|)\langle\tau \mid z\rangle} \alpha_{z} \hat{z}^{i}+\frac{1}{\langle\tau \mid z\rangle} \alpha_{z} z^{i} .
$$

The derivatives of $\lambda$ matrices are

$$
\begin{aligned}
& \partial_{j} \lambda^{i}=\frac{|\mathbf{z}|}{\langle\tau\rangle^{2}}\left[\frac{\hat{z}^{j} \alpha_{\perp}^{i}}{\langle\tau \mid z\rangle}+\frac{\tau^{2} \alpha_{\perp}^{j} \hat{z}^{i}}{\langle\tau \| z\rangle}+\left(\frac{\tau^{2} \delta_{\perp}^{j i}}{\langle\tau \| z\rangle}+\frac{\langle\tau\rangle \hat{z}^{j} \hat{z}^{i}}{\langle z\rangle}\right) \alpha_{z}\right], \\
& \partial_{i} \lambda^{i}=\frac{1}{\langle\tau\rangle^{2}}\left[\frac{2 \tau^{2}}{\langle\tau \| z\rangle}+\frac{\langle\tau\rangle}{\langle z\rangle}\right] \mathbf{z} \cdot \boldsymbol{\alpha},
\end{aligned}
$$

where

$$
\delta_{\perp}^{i j}=\delta^{i j}-\hat{z}^{i} \hat{z}^{j},
$$

and their estimates are easily obtained:

$$
\left|\lambda^{i}\right| \leq \text { const } \frac{\langle z\rangle}{\langle\tau\rangle}, \quad\left|\partial_{j} \lambda^{i}\right| \leq \text { const } \frac{|\mathbf{z}|}{\langle\tau\rangle\langle z\rangle}, \quad\left|\partial_{j} \partial_{i} \lambda^{k}\right| \leq \frac{\text { const }}{\langle\tau\rangle\langle z\rangle} .
$$

The $\mu$ matrix introduced in (11), and its derivative, take the form

$$
\mu=\frac{\langle\tau \mid z\rangle}{\langle\tau\rangle} \beta, \quad \partial_{i} \mu=\frac{z^{i}}{\langle\tau\rangle\langle\tau \mid z\rangle} \beta .
$$

The commutator, and the anti-commutator of matrices $\lambda^{i}$, the latter defining the form $\rho^{i j}$ introduced in (22), yield

$$
\begin{aligned}
{\left[\lambda^{i}, \lambda^{j}\right] } & =\frac{\langle\tau \mid z\rangle^{2}}{\langle\tau\rangle^{4}}\left[\alpha_{\perp}^{i}, \alpha_{\perp}^{j}\right]+2 \frac{\langle\tau \mid z\rangle\langle z\rangle}{\langle\tau\rangle^{3}} \alpha_{z}\left(\hat{z}^{i} \alpha_{\perp}^{j}-\hat{z}^{j} \alpha_{\perp}^{i}\right), \\
\rho^{i j} & =\frac{1}{2}\left[\lambda^{i}, \lambda^{j}\right]_{+}=-g_{\tau \tau} g^{i j}=\frac{\langle\tau \mid z\rangle^{2}}{\langle\tau\rangle^{4}} \delta_{\perp}^{i j}+\frac{\langle z\rangle^{2}}{\langle\tau\rangle^{2}} \hat{z}^{i} \hat{z}^{j} \\
& =\frac{1}{\langle\tau\rangle^{2}}\left[\left(1+\frac{|\mathbf{z}|^{2}}{\langle\tau\rangle^{2}}\right) \delta_{\perp}^{i j}+\langle z\rangle^{2} \hat{z}^{i} \hat{z}^{j}\right] .
\end{aligned}
$$

Using the latter form, we find for the $\tau$-derivative of $\rho$

$$
\dot{\rho}^{i j}=-\frac{2 \tau}{\langle\tau\rangle^{2}} \rho^{i j}-\frac{2 \tau|\mathbf{z}|^{2}}{\langle\tau\rangle^{6}} \delta_{\perp}^{i j},
$$


from which, in the sense of quadratic forms,

$$
\operatorname{sgn}(\tau) \dot{\rho}^{i j} \leq-\frac{2|\tau|}{\langle\tau\rangle^{2}} \rho^{i j},
$$

an inequality needed in Lemma 8. The expression first appearing in (45) and needed in the proof of Theorem 7 takes the form

$$
\partial_{i} \rho^{i j}=2 \frac{\tau^{2}+\langle\tau\rangle^{2}}{\langle\tau\rangle^{4}} z^{j} .
$$

The square root of the form $\rho$, introduced in (42), is explicitly

$$
s^{i j}=\frac{\langle\tau \mid z\rangle}{\langle\tau\rangle^{2}} \delta_{\perp}^{i j}+\frac{\langle z\rangle}{\langle\tau\rangle} \hat{z}^{i} \hat{z}^{j} .
$$

The quantity introduced in (39), and its properties, are:

$$
\begin{aligned}
\Lambda_{j} & =\frac{|\mathbf{z}|}{2\langle\tau \mid z\rangle}\left(\frac{\tau^{2}}{\langle\tau \| z\rangle}-\frac{\langle\tau\rangle\langle z\rangle}{\langle\tau \mid z\rangle^{2}}\right) i \alpha_{z} \alpha_{\perp}^{j}, \\
s^{i j} \Lambda_{j} & =\frac{|\mathbf{z}|}{2\langle\tau\rangle^{2}}\left(\frac{\tau^{2}}{\langle\tau \| z\rangle}-\frac{\langle\tau\rangle\langle z\rangle}{\langle\tau \mid z\rangle^{2}}\right) i \alpha_{z} \alpha_{\perp}^{i}, \\
\left|\Lambda_{j}\right| & \leq \frac{\langle\tau\rangle}{\langle\tau \mid z\rangle}, \quad\left|s^{i j} \Lambda_{j}\right| \leq \frac{1}{\langle\tau\rangle}, \quad\left|\dot{\Lambda}_{j}\right| \leq \frac{\text { const }}{\langle\tau \mid z\rangle}, \quad\left|s^{i j} \dot{\Lambda}_{j}\right| \leq \frac{\text { const }}{\langle\tau\rangle^{2}} .
\end{aligned}
$$

The calculation of $Q$ defined in (40) is more tedious, and we write down two of the intermediate steps:

$$
\begin{aligned}
\frac{1}{4} \partial_{j}\left(\left[\lambda^{j}, \partial \cdot \lambda\right]_{+}\right) & =\frac{\tau^{2}}{\langle\tau\rangle^{3}\langle\tau \| z\rangle}\left(2\langle z\rangle+\frac{|\mathbf{z}|^{2}}{\langle z\rangle}\right)+\frac{\tau^{2}}{\langle\tau\rangle^{2}\langle\tau \mid z\rangle\langle\tau \| z\rangle}+\frac{3}{2\langle\tau\rangle^{2}}, \\
\frac{1}{4}(\partial \cdot \lambda)^{2} & =\frac{\tau^{2}\langle z\rangle}{\langle\tau\rangle^{3}\langle\tau \| z\rangle}-\frac{\tau^{2}\langle\tau \mid z\rangle}{\langle\tau\rangle^{4}\langle\tau \| z\rangle}+\frac{\tau^{2}|\mathbf{z}|^{2}}{\langle\tau\rangle^{3}\langle z\rangle\langle\tau \| z\rangle}+\frac{|\mathbf{z}|^{2}}{4\langle\tau\rangle^{2}\langle z\rangle^{2}},
\end{aligned}
$$

which substituted in (40) gives

$$
\begin{aligned}
& 0<Q=\langle\tau\rangle^{-2}\left(\frac{\tau^{2}}{\langle\tau\rangle^{2}}+\frac{\tau^{2}}{\langle\tau \mid z\rangle\langle\tau \| z\rangle}+\frac{1}{4\langle z\rangle^{2}}+\frac{5}{4}\right) \leq \frac{\text { const }}{\langle\tau\rangle^{2}}, \\
& 0<N \leq \frac{\text { const }}{\langle\tau\rangle^{2}} .
\end{aligned}
$$

The following estimates are needed in the proof of Lemma 8

$$
s^{i j} \partial_{j} \mu=\frac{z^{i}\langle z\rangle}{\langle\tau\rangle^{2}\langle\tau \mid z\rangle} \beta, \quad\left|s^{i j} \partial_{j} \mu\right| \leq \frac{|\mathbf{z}|}{\langle\tau\rangle^{2}} .
$$

The matrix $\nu$ defined by (59), with the use of (48) is shown to satisfy the estimates

$$
|\nu| \leq \frac{\kappa}{\langle\tau\rangle}, \quad\left|s^{i j} \Lambda_{j} \nu\right| \leq \frac{\kappa}{\langle\tau\rangle^{2}},
$$

which are needed in the proof of Lemma 8. To simplify notation of its derivative, let us denote $\chi(u)=\xi(u)^{-1} \xi^{\prime}(u)$. Then

$$
\partial_{i} \nu=\frac{1}{\langle\tau\rangle}\left[\chi(\langle z\rangle) \alpha_{\perp}^{i}+\left(\chi(\langle z\rangle)+\frac{|\mathbf{z}|^{2}}{\langle z\rangle} \chi^{\prime}(\langle z\rangle)\right) \alpha_{z} \hat{z}^{i}\right],
$$




$$
s^{i j} \partial_{j} \nu=\frac{\langle\tau \mid z\rangle}{\langle\tau\rangle^{3}} \chi(\langle z\rangle) \alpha_{\perp}^{i}+\frac{1}{\langle\tau\rangle^{2}}\left(\langle z\rangle \chi(\langle z\rangle)+|\mathbf{z}|^{2} \chi^{\prime}(\langle z\rangle)\right) \alpha_{z} \hat{z}^{i} .
$$

It follows from $(48)$ that $\chi(u) \leq \kappa / u$ and $\left|\chi^{\prime}(u)\right| \leq$ const $/ u$, so

$$
\left|s^{i j} \partial_{j} \nu\right| \leq \operatorname{const} \frac{|\mathbf{z}|}{\langle\tau\rangle^{2}} .
$$

The spreading of the past and the future in Minkowski space, in the language of the coordinates $(\tau, \mathbf{z})$, is given by the following lemma. This result is needed for the description of scattering in Sect. 6 .

Lemma 15. The past (resp. future) of the set $\tau=\tau_{0},|\mathbf{z}| \leq r_{0}$ consists of the points $(\tau, \mathbf{z})$ such that $\tau<\tau_{0}$ (resp. $\tau>\tau_{0}$ ) and $|\mathbf{z}| \leq r$, where

$$
\begin{aligned}
r & =r_{0}\left[\left\langle\tau_{0}\right\rangle\langle\tau\rangle-\tau_{0} \tau\right]+\left\langle r_{0}\right\rangle\left|\tau\left\langle\tau_{0}\right\rangle-\langle\tau\rangle \tau_{0}\right|, \\
\langle r\rangle & =\left\langle r_{0}\right\rangle\left[\left\langle\tau_{0}\right\rangle\langle\tau\rangle-\tau_{0} \tau\right]+r_{0}\left|\tau\left\langle\tau_{0}\right\rangle-\langle\tau\rangle \tau_{0}\right| .
\end{aligned}
$$

It follows that

$$
\langle r\rangle \leq\left\langle r_{0}\right\rangle \begin{cases}\left(\left\langle\tau_{0}\right\rangle-\tau_{0}\right)(\langle\tau\rangle+\tau), & \tau \geq \tau_{0} \\ \left(\left\langle\tau_{0}\right\rangle+\tau_{0}\right)(\langle\tau\rangle-\tau), & \tau \leq \tau_{0}\end{cases}
$$

In particular, for $\tau_{0}=0$ and $\tau \in \mathbb{R}$ we have

$$
\langle r\rangle \leq 2\left\langle r_{0}\right\rangle\langle\tau\rangle \text {. }
$$

Proof. Simple geometrical analysis shows that the radius $r$ is given by the conditions that the vector $\left(\tau\langle r\rangle-\tau_{0}\left\langle r_{0}\right\rangle, 0,0,\langle\tau\rangle r-\left\langle\tau_{0}\right\rangle r_{0}\right)$ is lightlike and $\langle\tau\rangle r-\left\langle\tau_{0}\right\rangle r_{0}>0$, that is

$$
\epsilon\left(\tau\langle r\rangle-\tau_{0}\left\langle r_{0}\right\rangle\right)=\langle\tau\rangle r-\left\langle\tau_{0}\right\rangle r_{0}>0
$$

where $\epsilon=-1$ (resp. $\epsilon=+1$ ) for past (resp. future). We set $\tau_{0}=\sinh \left(T_{0}\right)$, $r_{0}=\sinh \left(R_{0}\right), \tau=\sinh (T), r=\sinh (R)$, where $R_{0}>0, R>0$. Then the equality above is easily transformed to $\sinh (R-\epsilon T)=\sinh \left(R_{0}-\epsilon T_{0}\right)$, so $R-R_{0}=\epsilon\left(T-T_{0}\right)$. Treating $R_{0}, T$ and $\left(T-T_{0}\right)$ as independent, we put $R=R_{0}+\epsilon\left(T-T_{0}\right)$ and $T_{0}=T-\left(T-T_{0}\right)$ in the inequality, which is then transformed to $\epsilon \sinh \left(T-T_{0}\right) \cosh \left(R_{0}+\epsilon T\right)>0$. Thus $\epsilon=\operatorname{sgn}\left(T-T_{0}\right)$ and $R=R_{0}+\left|T-T_{0}\right|$. Substituting this in $r=\sinh (R)$ and $\langle r\rangle=\cosh (R)$, one obtains (101). Moreover,

$$
\langle r\rangle \leq \cosh \left(R_{0}\right) \exp \left|T-T_{0}\right|= \begin{cases}\left\langle r_{0}\right\rangle \exp (T) \exp \left(-T_{0}\right), & T \geq T_{0}, \\ \left\langle r_{0}\right\rangle \exp \left(T_{0}\right) \exp (-T), & T \leq T_{0}\end{cases}
$$

which gives (102).

Finally, we state the following inequalities needed for the estimation of electromagnetic fields, discussed in Appendix $\mathrm{H}$.

Lemma 16. For $x=(\tau\langle z\rangle,\langle\tau\rangle \mathbf{z})$ the following holds:

$$
\begin{gathered}
\left|x^{0}\right|+|\mathbf{x}|+1 \geq\langle\tau\rangle\langle z\rangle, \\
|| x^{0}|-| \mathbf{x}||+1 \geq \frac{\langle\tau\rangle^{2}+\langle z\rangle^{2}}{2\langle\tau\rangle\langle z\rangle} .
\end{gathered}
$$


Proof. The first inequality follows from

$$
(|\tau|\langle z\rangle+\langle\tau\rangle|\mathbf{z}|+1)^{2} \geq|\tau|^{2}\langle z\rangle^{2}+\langle\tau\rangle^{2}|\mathbf{z}|^{2}+1 \geq\langle\tau\rangle^{2}\langle z\rangle^{2},
$$

and the second from

$$
\begin{aligned}
\| \tau|\langle z\rangle-\langle\tau\rangle| \mathbf{z}||+1 & =\frac{\left.\left|\tau^{2}\langle z\rangle^{2}-\langle\tau\rangle^{2}\right| \mathbf{z}\right|^{2} \mid}{|\tau|\langle z\rangle+\langle\tau\rangle|\mathbf{z}|}+1 \geq \frac{\left|\langle\tau\rangle^{2}-\langle z\rangle^{2}\right|+2\langle\tau\rangle\langle z\rangle}{2\langle\tau\rangle\langle z\rangle} \\
& \geq \frac{\left[\left(\langle\tau\rangle^{2}-\langle z\rangle^{2}\right)^{2}+4\langle\tau\rangle^{2}\langle z\rangle^{2}\right]^{\frac{1}{2}}}{2\langle\tau\rangle\langle z\rangle}=\frac{\langle\tau\rangle^{2}+\langle z\rangle^{2}}{2\langle\tau\rangle\langle z\rangle} .
\end{aligned}
$$

\section{Appendix E. Free Dirac Equation: The Fourier Representation}

Here, mainly to fix notation, we write down the integral Fourier form of the solution of the free Dirac equation for a given initial condition.

Let $H$ denote the unit hyperboloid $v^{2}=1, v^{0}>0$, with the Lorentzinvariant measure $\mathrm{d} \mu(v)=d^{3} v / v^{0}$. Moreover, introduce the projection operators in $\mathbb{C}^{4}$ defined by $P_{ \pm}(v)=\frac{1}{2}(\mathbf{1} \pm v \cdot \gamma)$. For $f \in \mathcal{S}\left(H, \mathbb{C}^{4}\right)$ the function

$$
\psi(x)=(2 \pi)^{-\frac{3}{2}} \int\left[e^{-i x \cdot v} P_{+}(v)-e^{i x \cdot v} P_{-}(v)\right] f(v) \mathrm{d} \mu(v)
$$

is a smooth solution of the Dirac equation with the initial condition

$$
\psi(0, \mathbf{x})=(2 \pi)^{-\frac{3}{2}} \int\left[e^{i \mathbf{x} \cdot \mathbf{v}} P_{+}(v)-e^{-i \mathbf{x} \cdot \mathbf{v}} P_{-}(v)\right] f(v) \mathrm{d} \mu(v)
$$

in the space $\mathcal{S}\left(\mathbb{R}^{3}, \mathbb{C}^{4}\right)$ (where $\mathbf{x} \cdot \mathbf{v}$ is the Euclidean product of the space parts of $x$ and $v$ ). The transformation

$$
\mathcal{S}\left(H, \mathbb{C}^{4}\right) \ni f \mapsto \mathcal{F} f=\psi(0, .) \in \mathcal{S}\left(\mathbb{R}^{3}, \mathbb{C}^{4}\right)
$$

extends to an isometric transformation from the Hilbert space $L_{\gamma}^{2}(H)$ of fourspinor functions with the scalar product $\left(f_{1}, f_{2}\right)=\int \overline{f_{1}(v)} \gamma \cdot v f_{2}(v) \mathrm{d} \mu(v)$ into $\mathcal{H}=\mathbb{C}^{4} \otimes L^{2}\left(\mathbb{R}^{3}\right)$. For $\psi(0, \mathbf{x}) \in \mathcal{S}\left(\mathbb{R}^{3}, \mathbb{C}^{4}\right)$ the inverse transformation is given by

$$
f(v)=(2 \pi)^{-\frac{3}{2}} \int\left[e^{-i \mathbf{v} \cdot \mathbf{x}} P_{+}(v)+e^{i \mathbf{v} \cdot \mathbf{x}} P_{-}(v)\right] \gamma^{0} \psi(0, \mathbf{x}) d^{3} x,
$$

which again extends to an isometric transformation on $\mathbb{C}^{4} \otimes L^{2}\left(\mathbb{R}^{3}\right)$. Therefore,

$$
\mathcal{F}: L_{\gamma}^{2}(H) \mapsto \mathcal{H}
$$

is a unitary transformation, and again $\mathcal{F}^{-1}: \mathcal{S}\left(\mathbb{R}^{3}, \mathbb{C}^{4}\right) \mapsto \mathcal{S}\left(H, \mathbb{C}^{4}\right)$. Thus, for $\varphi \in \mathcal{S}\left(\mathbb{R}^{3}, \mathbb{C}^{4}\right)$ the solution of the free Dirac equation with the initial condition $\psi(0, \mathbf{x})=\varphi(\mathbf{x})$ is represented by

$$
\psi(x)=(2 \pi)^{-\frac{3}{2}} \int\left[e^{-i x \cdot v} P_{+}(v)-e^{i x \cdot v} P_{-}(v)\right]\left[\mathcal{F}^{-1} \varphi\right](v) \mathrm{d} \mu(v) .
$$




\section{Appendix F. Solutions of the Wave Equation}

In this and the following two sections we shall use the language more extensively explained in [10], where also bibliographic sources are indicated. Moreover, we shall repeatedly make use of the following estimates, which we write down here for the convenience of the reader, and whose proof may be found in [8], Appendix B.

Lemma 17. Let $a>0, b \geq 0, c>0$ and $\alpha>0$. Then

$$
\int_{0}^{c}(a+b u)^{-\alpha} d u< \begin{cases}\frac{\alpha}{\alpha-1} \frac{c}{a^{\alpha-1}(a+b c)}, & \alpha>1, \\ \frac{1}{1-\alpha} \frac{c}{(a+b c)^{\alpha}}, & \alpha<1 .\end{cases}
$$

A large class of the solutions of the homogeneous wave equation may be represented in the following form

$$
\varphi(x)=-\frac{1}{2 \pi} \int \dot{F}(x \cdot l, l) d^{2} l,
$$

where $\dot{F}(s, l)=\partial_{s} F(s, l)$, with $F(s, l)$ a homogeneous of degree -1 function of a real variable $s$ and null (light-like), future-pointing vectors $l$. Consequently, $\dot{F}(s, l)$ is homogeneous of degree -2 , and at this stage this function is all one needs for the above representation, but later the function $F$ will play a role. For $\dot{F}$ in the class $C^{0}$ the field $\varphi$ is in $C^{0}$ and satisfies the wave equation in the distributional sense. For $F$ in the class $C^{3}$, the field is in the class $C^{2}$ and the wave equation is satisfied in the direct sense. The measure $d^{2} l$ is the Lorentz invariant measure on the set of null directions, applicable to functions of $l$, homogeneous of degree -2 . If $f(l)$ is such function, and $\left(e_{0}, \ldots, e_{3}\right)$ is any Minkowski basis, then

$$
\int f(l) d^{2} l=\int f(k) \mathrm{d} \Omega(\mathbf{k}),
$$

where $k=l / l^{0}, \mathrm{~d} \Omega(\mathbf{k})$ is the spherical angle measure in this coordinate system, and the value of this integral does not depend on the choice of basis. The operators of intrinsic differentiation in the cone are given by $L_{a b}=l_{a}\left(\partial / \partial l^{b}\right)-l_{b}\left(\partial / \partial l^{a}\right)$, and satisfy

$$
\int L_{a b} f(l) d^{2} l=0
$$

Let $Z^{a}(l)$ be a vector function, such that $l \cdot Z(l)=0$. If one extends $Z(l)$ to the neighborhood of the cone with the preservation of this property, then

$$
L_{0 a}\left[Z^{a}(l) / l^{0}\right]=\partial \cdot Z(l),
$$

independently of any particular extension, and where after differentiation the rhs is restricted to the cone. Therefore, the rhs is well defined and independent of the choice of basis. Moreover, let in addition $Z(l)$ be homogeneous of degree -1 , then

$$
\int \partial \cdot Z(l) d^{2} l=0
$$


We shall use the coordinates $(u, \phi)$ defined by

$$
l=l^{0} k, \quad k=e_{0}+e_{3} u+\sqrt{1-u^{2}} R(\phi), \quad R(\phi)=e_{1} \cos \phi+e_{2} \sin \phi .
$$

We note for later use that

$$
\ddot{R}(\phi) \equiv \partial_{\phi}^{2} R(\phi)=-R(\phi) .
$$

We choose $e_{3}=\mathbf{x} /|\mathbf{x}|$, and then

$$
\varphi(x)=-\frac{1}{2 \pi} \int F\left(x^{0}-|\mathbf{x}| u, k(u, \phi)\right) \mathrm{d} u \mathrm{~d} \phi .
$$

The following theorem gives the properties of decay in spacetime of the solutions (105). In the estimates (110), (112), (113) below, and other of similar type to appear later, one should understand that vectors $l$ are scaled to $l^{0}=1$. The form of such estimates does not depend on the choice of a basis, only the bounding constants change. Recall that $\theta$ is the Heaviside step function.

Theorem 18. Let $\varphi(x)$ be represented by formula (105), with $\dot{F}(s, l)$ and $F(s, l)$ as described there, and let $\varepsilon \in(0,1)$.

(i) If $\dot{F}(s, l)$ is of class $C^{0}$, and for some $n \in\{0,1, \ldots\}$ is bounded by

$$
|\dot{F}(s, l)| \leq \frac{\text { const }}{(|s|+1)^{n+\varepsilon}},
$$

then $\varphi(x)$ is a $C^{0}$ field and the following estimates are satisfied

$$
|\varphi(x)| \leq \mathrm{const} \begin{cases}\frac{1}{(|x|+1)^{\varepsilon}}, & n=0, \\ \frac{1}{|x|+1}\left[\frac{\theta\left(x^{2}\right)}{\left(\left|x^{0}\right|-|\mathbf{x}|+1\right)^{n-1+\varepsilon}}+\theta\left(-x^{2}\right)\right], & n \geq 1 .\end{cases}
$$

(ii) If $F(s, l)$ is of class $C^{1}$, and for some $n \in\{1,2, \ldots\}$ is bounded by

$$
\left|L^{\alpha} F(s, l)\right| \leq \frac{\text { const }}{(|s|+1)^{n-1+\varepsilon}}, \quad|\alpha| \leq 1,
$$

then $\varphi(x)$ is a $C^{0}$ field and the following stronger estimates hold

$$
\begin{aligned}
|\varphi(x)| & \leq \frac{\text { const }}{(|x|+1)\left(|| x^{0}|-| \mathbf{x}||+1\right)^{\varepsilon}}, \quad \varepsilon<\frac{1}{2}, \quad n=1, \\
|\varphi(x)| & \leq \frac{\text { const }}{|x|+1}\left[\frac{1}{\left(|| x^{0}|-| \mathbf{x}||+1\right)^{n-1+\varepsilon}}+\frac{\theta\left(-x^{2}\right)}{(|x|+1)^{\frac{1}{2}}\left(|\mathbf{x}|-\left|x^{0}\right|+1\right)^{\frac{1}{2}}}\right], \\
n & \geq 2 .
\end{aligned}
$$

(iii) If $F(s, l)$ is of class $C^{2}$, and for some $n \in\{1,2, \ldots\}$ is bounded by

$$
\left|L^{\alpha} F(s, l)\right| \leq \frac{\text { const }}{(|s|+1)^{n-1+\varepsilon}}, \quad|\alpha| \leq 2,
$$


then $\varphi(x)$ is a $C^{1}$ field and the following stronger estimates hold

$$
|\varphi(x)| \leq \begin{cases}\frac{\text { const }}{(|x|+1)\left(|| x^{0}|-| \mathbf{x}||+1\right)^{\varepsilon}}, & n=1, \\ \frac{\text { const }}{|x|+1}\left[\frac{1}{\left(|| x^{0}|-| \mathbf{x}||+1\right)^{n-1+\varepsilon}}+\frac{\theta\left(-x^{2}\right)}{|x|+1}\right], & n \geq 2 .\end{cases}
$$

Note that due to the properties of homogeneity of $\dot{F}$ and $F$, the bound (110) is satisfied in all three cases (with $n \geq 2$ in (ii) and $n \geq 1$ in (iii)). Case (ii) for $n=1$ could also be treated, but the result is more involved, and we do not need it anyway.

Proof. (i) Using the bound (110) and the integral (109), after the change of variables $\sigma=\operatorname{sgn}\left(x^{0}\right)\left(x^{0}-|\mathbf{x}| u\right)$, we obtain

$$
\begin{aligned}
|\varphi(x)| & \leq \frac{\text { const }}{|\mathbf{x}|} \int_{\left|x^{0}\right|-|\mathbf{x}|}^{\left|x^{0}\right|+|\mathbf{x}|} \frac{\mathrm{d} \sigma}{(|\sigma|+1)^{n+\varepsilon}} \\
& \leq \frac{\text { const }}{|\mathbf{x}|} \begin{cases}\int_{0}^{2|\mathbf{x}|} \frac{\mathrm{d} \xi}{\left(\xi+\left|x^{0}\right|-|\mathbf{x}|+1\right)^{n+\varepsilon}}, & x^{2} \geq 0, \\
\int_{0}^{\left|x^{0}\right|+|\mathbf{x}|} \frac{\mathrm{d} \sigma}{(\sigma+1)^{n+\varepsilon}}, & x^{2} \leq 0 .\end{cases}
\end{aligned}
$$

Lemma 17 gives now the bounds (111).

(ii) and (iii) These cases give more restrictive bounds only in the region $x^{2} \leq 0,|\mathbf{x}| \geq 1$, in which $|\mathbf{x}| \geq \operatorname{const}(|x|+1)$. We use the equalities (114) and (115), respectively, of Lemma 19 below. To shorten notation, we denote the exponent in (112) and (113) by $q=n-1+\varepsilon$. For $x^{2} \leq 0$ we then have

$$
|\varphi(x)| \leq \frac{\text { const }}{|\mathbf{x}|}\left[\frac{1}{\left(|\mathbf{x}|-\left|x^{0}\right|+1\right)^{q}}+\int_{-1}^{1}\left\{\begin{array}{c}
\left(1-u^{2}\right)^{-\frac{1}{2}} \\
1
\end{array}\right\} \frac{d u}{\left(|| x^{0}|+| \mathbf{x}|u|+1\right)^{q}}\right]
$$

where $\left(1-u^{2}\right)^{-\frac{1}{2}}$ applies in case (ii) and 1 in case (iii). Integration in case (iii) is done as in case (i) and the thesis of this case follows. The integral in case (ii), after the substitution $1-u=t$, is bounded by 2 times the integral

$$
\begin{aligned}
& \int_{0}^{1} \frac{d t}{f(a, b, t)}, \quad f(a, b, t)=t^{\frac{1}{2}}(|b t-a|+1)^{q}, \\
& 0 \leq a=|\mathbf{x}|-\left|x^{0}\right| \leq|\mathbf{x}|=b \geq \operatorname{const}(|x|+1) .
\end{aligned}
$$

For $t \in[0, a /(2 b)]$ we have $f(a, b, t) \geq$ const $t^{\frac{1}{2}}(a+1)^{q}$, so

$$
\int_{0}^{\frac{a}{2 b}} \frac{d t}{f(a, b, t)} \leq \text { const } b^{-\frac{1}{2}}(a+1)^{\frac{1}{2}-q}
$$

For $t \in[a /(2 b), 2 a / b]$ we have $f(a, b, t) \geq$ const $a^{\frac{1}{2}} b^{-\frac{1}{2}}(|b t-a|+1)^{q}$. The integral is now split into intervals $[a /(2 b), a / b]$ and $[a / b, 2 a / b]$ and evaluated 
with the use of Lemma 17. This gives

$$
\int_{\frac{a}{2 b}}^{\frac{2 a}{b}} \frac{d t}{f(a, b, t)} \leq \text { const } b^{-\frac{1}{2}} \times \begin{cases}(a+1)^{\frac{1}{2}-q}, & q<1 \\ (a+1)^{-\frac{1}{2}}, & q>1 .\end{cases}
$$

Finally, if $2 a / b \leq 1$, then for $t \in[2 a / b, 1]$ we have $f(a, b, 2 a / b+s) \geq$ const $s^{\frac{1}{2}}(a+1+b s)^{q}$, and by changing further the integration variable to $r=b(a+1)^{-1} s$ one finds

$$
\int_{\frac{2 a}{b}}^{1} \frac{d t}{f(a, b, t)} \leq \mathrm{const} \times \begin{cases}b^{-q}, & q<\frac{1}{2}, \\ b^{-\frac{1}{2}}(a+1)^{\frac{1}{2}-q}, & q>\frac{1}{2} .\end{cases}
$$

Substituting the values of $a$ and $b$ and noting that $b^{-\frac{1}{2}}(a+1)^{\frac{1}{2}-q} \leq$ const $b^{-q}$ for $q<\frac{1}{2}, b \geq 1$, one obtains the thesis.

Lemma 19. Let $F(s, l)$ be homogeneous of degree -1 and choose $e_{3}$ in (107) as $e_{3}=\mathbf{x} /|\mathbf{x}|$. Then the following identities hold

$$
\begin{aligned}
\frac{1}{2 \pi} & \int \dot{F}(x \cdot l, l) d^{2} l+\frac{1}{|\mathbf{x}|}\left[F\left(x^{0}-|\mathbf{x}|,(1, \mathbf{x} /|\mathbf{x}|)\right)-F\left(x^{0}+|\mathbf{x}|,(1,-\mathbf{x} /|\mathbf{x}|)\right)\right] \\
& =\frac{1}{2 \pi|\mathbf{x}|} \int\left(l^{0}\right)^{-1}\left[L_{03}^{\prime}-\frac{u}{\sqrt{1-u^{2}}} R^{i} L_{0 i}^{\prime}\right] F(x \cdot l, l) d^{2} l \\
& =\frac{1}{2 \pi|\mathbf{x}|} \int\left(l^{0}\right)^{-1}\left[L_{03}^{\prime}-u \dot{R}^{i} \dot{R}^{j} L_{0 i}^{\prime} L_{0 j}^{\prime}\right] F(x \cdot l, l) d^{2} l
\end{aligned}
$$

where prime at $L_{a b}^{\prime}$ indicates that these operators act only on the second argument in $F(x \cdot l, l)$. The equalities (114) and (115) hold for $F$ of class $C^{1}$ and $C^{2}$, respectively.

Proof. Using the form (107), we find

$$
\partial_{u}=\frac{1}{l^{0}} \frac{\partial l^{a}}{\partial u} L_{0 a}=L_{03}-\frac{u}{\sqrt{1-u^{2}}} R^{i} L_{0 i} .
$$

We apply this identity to $F(s, l)$, then set $s=x \cdot l$ and divide by $l^{0}$. As $l^{0}$ does not depend on $u$, the result may be written as

$$
\partial_{u}\left[\left(l^{0}\right)^{-1} F(x \cdot l, l)\right]+|\mathbf{x}| \dot{F}(x \cdot l, l)=\left(l^{0}\right)^{-1}\left[L_{03}^{\prime}-\frac{u}{\sqrt{1-u^{2}}} R^{i} L_{0 i}^{\prime}\right] F(x \cdot l, l),
$$

(primes at $L^{\prime}$ as in the thesis). We integrate this identity over l's. The first term on the lhs gives

$$
\begin{aligned}
& \int \partial_{u} F\left(x^{0}-|\mathbf{x}| u, k(u, \phi)\right) \mathrm{d} u \mathrm{~d} \phi \\
& \quad=2 \pi\left[F\left(x^{0}-|\mathbf{x}|,(1, \mathbf{x} /|\mathbf{x}|)\right)-F\left(x^{0}+|\mathbf{x}|,(1,-\mathbf{x} /|\mathbf{x}|)\right)\right] .
\end{aligned}
$$

Dividing by $2 \pi|\mathbf{x}|$ and rearranging the terms, we obtain equality (114). Next, using (108) we transform the second term of the integrand as

$$
\begin{aligned}
- & \frac{u}{l^{0} \sqrt{1-u^{2}}} R^{i} L_{0 i}^{\prime} F(x \cdot l, l)=\partial_{\phi}\left[\frac{u}{l^{0} \sqrt{1-u^{2}}} \dot{R}^{i} L_{0 i}^{\prime} F(x \cdot l, l)\right] \\
& -\frac{u}{l^{0} \sqrt{1-u^{2}}} \dot{R}^{i} \partial_{\phi}^{\prime}\left[L_{0 i}^{\prime} F(x \cdot l, l)\right]
\end{aligned}
$$


where we have taken into account that $l^{0}$ and $x \cdot l$ do not depend on $\phi$. The first term on the rhs vanishes when integrated, while in the second term we use another differential identity:

$$
\partial_{\phi}=\frac{1}{l^{0}} \frac{\partial l^{a}}{\partial \phi} L_{0 a}=\sqrt{1-u^{2}} \dot{R}^{a} L_{0 a} .
$$

Substituting the result into the integral, we obtain (115).

\section{Appendix G. Sources}

Let $\rho(x)$ be a continuous field on Minkowski space. We formulate two decay conditions, with some fixed numbers $\omega>0$ and $\varepsilon \in(0,1)$ :

$$
\begin{array}{ll}
\text { (A) } \quad|\rho(x)| \leq \frac{\text { const }}{(|x|+1)^{\omega}}, \\
\text { (B) }|\rho(x)| \leq \frac{\text { const }}{(|x|+1)^{3}}\left[\theta\left(x^{2}\right)+\frac{1}{(|x|+1)^{\varepsilon}}\right] .
\end{array}
$$

We want to consider the existence and decay properties of the advanced and retarded solutions

$$
\varphi_{\text {ret/adv }}(x)=4 \pi \int D_{\text {ret/adv }}(x-y) \rho(y) d y
$$

of the equation

$$
\square \varphi=4 \pi \rho,
$$

and of their difference, the radiation field

$$
\varphi_{\mathrm{rad}}(x)=\varphi_{\mathrm{ret}}(x)-\varphi_{\mathrm{adv}}(x)=4 \pi \int D(x-y) \rho(y) \mathrm{d} y=-\frac{1}{2 \pi} \int \mathrm{d} V(x \cdot l, l) d^{2} l,
$$

where (see [10])

$$
V(s, l)=\int \delta(s-x \cdot l) \rho(x) \mathrm{d} x .
$$

We use the standard notation

$$
\begin{aligned}
& D_{\text {ret } / \text { adv }}(x)=\frac{1}{2 \pi} \theta\left( \pm x^{0}\right) \delta\left(x^{2}\right), \\
& D(x)=D_{\text {ret }}(x)-D_{\text {adv }}(x)=\frac{1}{2 \pi} \operatorname{sgn}\left(x^{0}\right) \delta\left(x^{2}\right) .
\end{aligned}
$$

Lemma 20. (i) If $\rho(x)$ satisfies condition (A) for $\omega>2$, then $\varphi_{\mathrm{adv} / \mathrm{ret}}(x)$ are well defined and continuous, and the following bound is satisfied:

$$
\left|\varphi_{\text {adv }}(x)\right| \leq \frac{\text { const }}{(|x|+1)^{\omega-2}} \quad \text { for } \quad x^{0} \geq 0 ;
$$

similarly for the retarded solution in the half-space $x^{0} \leq 0$.

(ii) If in addition to (i) $\rho(x)$ is of class $C^{1}$, and $\nabla \rho(x)$ and $x \cdot \nabla \rho(x)$ satisfy condition (A) for $\omega>2$, then $\varphi_{\mathrm{adv} / \mathrm{ret}}(x)$ are of class $C^{1}$ and

$$
x \cdot \nabla \varphi_{\mathrm{adv} / \mathrm{ret}}(x)=\int D_{\mathrm{adv} / \mathrm{ret}}(x-y)(y \cdot \nabla+2) \rho(y) d y .
$$


(iii) If $\rho(x)$ satisfies condition (A) for $\omega>3$, or condition (B), then $V(s, l)$ is well defined and continuous and satisfies the bound

$$
|V(s, l)| \leq \frac{\text { const }}{(|s|+1)^{\omega-3}},
$$

where $\omega=3$ in case of condition $(B)$.

(iv) If in addition to (iii) $\rho(x)$ is of class $C^{1}$, and $\nabla \rho(x)$ and $x \cdot \nabla \rho(x)$ satisfy condition (A) for $\omega>3$ or condition $(B)$, then $V(s, l)$ is of class $C^{1}$ and

$$
s \partial_{s} \int \delta(s-x \cdot l) \rho(x) d x=\int \delta(s-x \cdot l)(x \cdot \nabla+3) \rho(x) d x .
$$

Proof. (i) The use of the integral formula (118) and the bound (116) gives

$$
\left|\varphi_{\mathrm{adv}}(x)\right|=\left|\int \frac{\rho\left(x^{0}+|\mathbf{z}|, \mathbf{x}+\mathbf{z}\right)}{|\mathbf{z}|} d^{3} z\right| \leq \operatorname{const} \int_{0}^{\infty} I\left(x^{0},|\mathbf{x}|, r\right) \mathrm{d} r,
$$

where we have denoted $r=|\mathbf{z}|$, and

$$
I\left(x^{0},|\mathbf{x}|, r\right)=\int_{0}^{2} \frac{r d u}{\left[1+\left(r+x^{0}\right)^{2}+(r-|\mathbf{x}|)^{2}+2|\mathbf{x}| r u\right]^{\frac{\omega}{2}}}
$$

with $u=\cos [\measuredangle(\mathbf{z}, \mathbf{x})]+1$. Using the estimate (104), we find

$$
I\left(x^{0},|\mathbf{x}|, r\right) \leq \frac{\text { const } r}{\left[1+\left(r+x^{0}\right)^{2}+(r+|\mathbf{x}|)^{2}\right]\left[1+\left(r+x^{0}\right)^{2}+(r-|\mathbf{x}|)^{2}\right]^{\frac{\omega}{2}-1}},
$$

so the integral on the rhs of (122) is well defined, and the continuity of $\varphi_{\text {adv }}(x)$ easily follows. Moreover, for $x^{0} \geq 0$ it follows

$$
I\left(x^{0},|\mathbf{x}|, r\right) \leq \frac{\text { const } r}{\left[1+x^{0}+|\mathbf{x}|+r\right]^{2}\left[1+r+x^{0}+|r-| \mathbf{x}||\right]^{\omega-2}},
$$

and then

$$
\int_{0}^{|\mathbf{x}|} I\left(x^{0},|\mathbf{x}|, r\right) \mathrm{d} r \leq \frac{\text { const }}{\left[1+x^{0}+|\mathbf{x}|\right]^{\omega}} \int_{0}^{|\mathbf{x}|} r \mathrm{~d} r \leq \frac{\text { const }}{(|x|+1)^{\omega-2}},
$$

and (with $\sigma=r-|\mathbf{x}|$ )

$$
\int_{|\mathbf{x}|}^{\infty} I\left(x^{0},|\mathbf{x}|, r\right) \mathrm{d} r \leq \mathrm{const} \int_{0}^{\infty} \frac{\mathrm{d} \sigma}{\left(\sigma+x^{0}+|\mathbf{x}|+1\right)^{\omega-1}} \leq \frac{\text { const }}{(|x|+1)^{\omega-2}}
$$

which ends the proof of (i).

(ii) We integrate the identity

$$
\begin{aligned}
\frac{x \cdot \nabla_{x} \rho\left(x^{0}+|\mathbf{z}|, \mathbf{x}+\mathbf{z}\right)}{|\mathbf{z}|}= & \frac{\left(y \cdot \nabla_{y}+2\right) \rho(y) \mid y=\left(x^{0}+|\mathbf{z}|, \mathbf{x}+\mathbf{z}\right)}{|\mathbf{z}|} \\
& -\frac{\partial}{\partial z^{i}}\left(\frac{z^{i} \rho\left(x^{0}+|\mathbf{z}|, \mathbf{x}+\mathbf{z}\right)}{|\mathbf{z}|}\right)
\end{aligned}
$$

over $|\mathbf{z}| \leq R$ and transform the integral of the second term on the rhs to a surface integral. This surface contribution is bounded by $R I\left(x^{0},|\mathbf{x}|, R\right)$, so it vanishes in the limit $R \rightarrow \infty$. Formula (119) follows. 
(iii) We choose the space basis in which $e_{3}=1$, introduce new variables $\alpha=x^{0}-x^{3}, \beta=x^{0}+x^{3}$, and denote $\mathbf{x}_{\perp}=x^{1} e_{1}+x^{2} e_{2}$. Conditions (116) and (117) may be then written as

$$
\begin{aligned}
& |\rho(x)| \leq \frac{\text { const }}{\left(1+\alpha^{2}+\beta^{2}+\left|\mathbf{x}_{\perp}\right|^{2}\right)^{\frac{\omega}{2}}}, \\
& |\rho(x)| \leq \frac{\text { const } \theta\left(\alpha \beta-\left|\mathbf{x}_{\perp}\right|^{2}\right)}{\left(1+\alpha^{2}+\beta^{2}+\left|\mathbf{x}_{\perp}\right|^{2}\right)^{\frac{3}{2}}}+\frac{\text { const }}{\left(1+\alpha^{2}+\beta^{2}+\left|\mathbf{x}_{\perp}\right|^{2}\right)^{\frac{3+\varepsilon}{2}}},
\end{aligned}
$$

and $V$ takes the form $V(s, l)=\frac{1}{2} \int \rho\left(\alpha=s, \beta, \mathbf{x}_{\perp}\right) \mathrm{d} \beta d^{2} x_{\perp}$. For the case (A) with $\omega>3$ we then have

$$
\begin{aligned}
|V(s, l)| & \leq \operatorname{const} \int \frac{\mathrm{d} \beta d^{2} x_{\perp}}{\left(1+s^{2}+\beta^{2}+\left|\mathbf{x}_{\perp}\right|^{2}\right)^{\frac{\omega}{2}}} \\
& \leq \operatorname{const} \int_{0}^{\infty} \frac{r^{2} \mathrm{~d} r}{(1+|s|+r)^{\omega}}=\frac{\text { const }}{(1+|s|)^{\omega-3}} .
\end{aligned}
$$

For the case (B) it is now sufficient to restrict integral to the region $x^{2} \geq 0$, which leads to the estimation of the integral $\int K(\beta) \mathrm{d} \beta$, with

$$
\begin{aligned}
K(\beta) & =\int \frac{\theta\left(s \beta-\left|\mathbf{x}_{\perp}\right|^{2}\right) d^{2} x_{\perp}}{\left(1+s^{2}+\beta^{2}+\left|\mathbf{x}_{\perp}\right|^{2}\right)^{\frac{3}{2}}}=\operatorname{const} \theta(s \beta) \int_{0}^{s \beta} \frac{\mathrm{d} \xi}{\left(1+s^{2}+\beta^{2}+\xi\right)^{\frac{3}{2}}} \\
& \leq \operatorname{const} \frac{\theta(s \beta) s \beta}{\left(1+s^{2}+\beta^{2}\right)^{\frac{3}{2}}} \leq \operatorname{const} \frac{\theta(s \beta)|s|}{(1+|s|+|\beta|)^{2}} .
\end{aligned}
$$

This leads to

$$
\int K(\beta) \mathrm{d} \beta \leq \text { const }|s| \int_{0}^{\infty} \frac{\mathrm{d} \sigma}{(1+|s|+\sigma)^{2}} \leq \text { const } .
$$

(iv) We integrate the identity

$$
\begin{aligned}
s \partial_{s} \rho\left(s, \beta, \mathbf{x}_{\perp}\right)= & (x \cdot \nabla+3) \rho(x)_{\mid \alpha=s} \\
& -\partial_{\beta}\left(\beta \rho\left(s, \beta, \mathbf{x}_{\perp}\right)\right)-\sum_{i=1}^{2} \partial_{x^{i}}\left(x^{i} \rho\left(s, \beta, \mathbf{x}_{\perp}\right)\right)
\end{aligned}
$$

over the region $\left|\mathbf{x}_{\perp}\right| \leq R,|\beta| \leq B$. The contribution of the surface terms is bounded by

$$
\sum_{\epsilon= \pm} B \int_{\mathbb{R}^{2}}\left|\rho\left(s, \pm B, \mathbf{x}_{\perp}\right)\right| d^{2} x_{\perp}+R^{2} \int_{\mathbb{R} \times S^{1}}|\rho(s, \beta, R \mathbf{n})| \mathrm{d} \beta \mathrm{d} \Omega(\mathbf{n}),
$$

where $\mathrm{d} \Omega(\mathbf{n})$ is the angle measure on the unit circle $S^{1}$. Condition (A) with $\omega>3$ is stronger than condition (B), so it is sufficient to consider the latter, in the form given in the proof of (iii) above. Then, the first contribution in (123) is bounded by

$$
\begin{aligned}
& \text { const }\left[\int_{0}^{|s| B} \frac{B \mathrm{~d} \zeta}{\left(1+s^{2}+B^{2}+\zeta\right)^{\frac{3}{2}}}+\int_{0}^{\infty} \frac{B \mathrm{~d} \zeta}{\left(1+s^{2}+B^{2}+\zeta\right)^{\frac{3+\epsilon}{2}}}\right] \\
& \quad \leq \frac{\operatorname{const}|s| B^{2}}{(1+|s|+B)^{3}}+\frac{\operatorname{const} B}{(1+|s|+B)^{1+\epsilon}},
\end{aligned}
$$


where we have substituted $\left|\mathbf{x}_{\perp}\right|^{2}=\zeta$. The second term in (123) is bounded by

$$
\begin{gathered}
\text { const }\left[\int_{R^{2} /|s|}^{\infty} \frac{R^{2} \mathrm{~d} \beta}{(1+|s|+R+\beta)^{3}}+\int_{0}^{\infty} \frac{R^{2} \mathrm{~d} \beta}{(1+|s|+R+\beta)^{3+\epsilon}}\right] \\
\leq \frac{\operatorname{const} R^{2}}{\left(1+|s|+R+R^{2} /|s|\right)^{2}}+\frac{\operatorname{const} R^{2}}{(1+|s|+R)^{2+\epsilon}} .
\end{gathered}
$$

All these bounds vanish in the limit $B, R \rightarrow \infty$, which leads to the identity (121).

\section{Appendix H. Electromagnetic Fields and Lorenz Potentials}

We apply now the results of the discussion of the last two sections to the case of electromagnetic fields. We start with the Lorenz potentials of free fields, which we assume to have been produced as radiation fields by some conserved currents.

Theorem 21. Let $J(x)$ satisfy Assumption III in Sect. 7. Then, the Lorenz potential $A_{\mathrm{rad}}(x)$ of the radiated field of this current and the scalar field $C_{\text {rad }}(x)=x \cdot A_{\text {rad }}(x)$ satisfy the following decay estimates:

$$
\begin{aligned}
& \left.\begin{array}{l}
\left|A_{\text {rad }}(x)\right| \\
\left|\nabla C_{\text {rad }}(x)\right|
\end{array}\right\} \leq \frac{\text { const }}{|x|+1}\left[\frac{1}{\left(|| x^{0}|-| \mathbf{x}||+1\right)^{\varepsilon}}+\theta\left(-x^{2}\right)\right] \\
& \left|\nabla A_{\text {rad }}(x)\right| \leq \frac{\text { const }}{|x|+1}\left[\frac{1}{\left(|| x^{0}|-| \mathbf{x}||+1\right)^{1+\varepsilon}}+\frac{\theta\left(-x^{2}\right)}{|x|+1}\right], \\
& \left|\nabla \nabla C_{\text {rad }}(x)\right| \leq \frac{\text { const }}{|x|+1}\left[\frac{1}{\left(|| x^{0}|-| \mathbf{x}||+1\right)^{1+\varepsilon}}+\frac{\theta\left(-x^{2}\right)}{(|x|+1)^{\frac{1}{2}}\left(|\mathbf{x}|-\left|x^{0}\right|+1\right)^{\frac{1}{2}}}\right], \\
& \left|\nabla \nabla A_{\text {rad }}(x)\right| \leq \frac{\text { const }}{|x|+1}, \\
& \left|(x \cdot \nabla+1) A_{\text {rad }}(x)\right| \\
& \left|\nabla\left(x \cdot \nabla C_{\text {rad }}\right)(x)\right| \leq \frac{\text { const }}{(|x|+1)\left(|| x^{0}|-| \mathbf{x}||+1\right)^{\varepsilon}}, \\
& \left|(x \cdot \nabla)^{k} C_{\text {rad }}(x)\right| \leq \frac{\text { const }}{(|x|+1)^{\varepsilon}}, \quad k=1,2 .
\end{aligned}
$$

The estimate (127) could be refined, but this is not needed for our purposes.

Proof. It follows from the assumption (70) and Lemma 20 (iii) that the function

$$
V(s, l)=\int \delta(s-x \cdot l) J(x) d x=\int J\left(\frac{s+\mathbf{x} \cdot \mathbf{l}}{l^{0}}, \mathbf{x}\right) \frac{d^{3} x}{l^{0}}
$$

is of class $C^{3}$. The use of estimate (71), relation (121) and the estimates of the form (120) shows that

$$
\left|\partial_{s}^{k} L^{\alpha} \dot{V}(s, l)\right| \leq \frac{\text { const }}{(|s|+1)^{k+1+\varepsilon}}, \quad k+|\alpha| \leq 2 .
$$


From the representation

$$
A_{\text {rad }}(x)=-\frac{1}{2 \pi} \int \dot{V}(x \cdot l, l) d^{2} l,
$$

by the use of the calculation

$$
\partial \cdot V(x \cdot l, l)=x \cdot \dot{V}(x \cdot l, l)+\partial^{\prime} \cdot V(x \cdot l, l)
$$

and the integral identity (106), we also find

$$
\begin{aligned}
C_{\mathrm{rad}}(x) & =\frac{1}{2 \pi} \int \partial^{\prime} \cdot V(x \cdot l, l) d^{2} l, \\
\nabla_{a} C_{\mathrm{rad}}(x) & =\frac{1}{2 \pi} \int l_{a} \partial^{\prime} \cdot \dot{V}(x \cdot l, l) d^{2} l .
\end{aligned}
$$

(Similarly as $L_{a b}^{\prime}$ in Lemma 19 in Appendix F, $\partial^{\prime}$ acts only on the second argument.) All estimates now follow from the appropriate statements of Theorem 18: estimates (124) from (i) with $n=1$, estimate (125) from (iii) for $n=2$, estimate (126) from (ii) for $n=2$, estimate (127) from (i) with $n=1$. Next, with $W(s, l)=s \dot{V}(s, l)$, we have

$$
\begin{aligned}
(x \cdot \nabla+1) A_{\mathrm{rad}}(x) & =-\frac{1}{2 \pi} \int \dot{W}(x \cdot l, l) d^{2} l, \\
\nabla_{a}\left(x \cdot \nabla C_{\mathrm{rad}}(x)\right) & =\frac{1}{2 \pi} \int l_{a} \partial^{\prime} \cdot \dot{W}(x \cdot l, l) d^{2} l,
\end{aligned}
$$

so the estimates (128) follow from Theorem 18 (ii) with $n=1$. Finally, with $U(s, l)=s \dot{W}(s, l)$, we have

$$
\begin{aligned}
x \cdot \nabla C_{\mathrm{rad}}(x) & =\frac{1}{2 \pi} \int \partial^{\prime} \cdot W(x \cdot l, l) d^{2} l, \\
(x \cdot \nabla)^{2} C_{\mathrm{rad}}(x) & =\frac{1}{2 \pi} \int \partial^{\prime} \cdot U(x \cdot l, l) d^{2} l,
\end{aligned}
$$

so the estimates (129) follow from Theorem 18 (i) for $n=0$.

Free in/out fields of the type discussed above are now augmented by ret/adv fields to produce the total electromagnetic field.

Theorem 22. Let the electromagnetic potential A satisfy Assumption III in Sect. 7. Then, the following estimates are satisfied:

$$
\begin{aligned}
\left|\nabla^{\alpha} A(x)\right| & \leq \frac{\text { const }}{|\nabla C(x)|} \leq \frac{\text { const }}{\langle\tau\rangle\langle z\rangle}, \quad|\alpha|=0,2 \\
|\nabla A(x)| & \leq \frac{\text { const }}{|x|+1}\left[\frac{1}{\left(|| x^{0}|-| \mathbf{x}||+1\right)^{1+\varepsilon}}+\frac{1}{|x|+1}\right] \\
& \leq \frac{\text { const }}{\langle\tau\rangle\langle z\rangle}\left[\frac{1}{\langle\tau\rangle\langle z\rangle}+\left(\frac{\langle\tau\rangle\langle z\rangle}{\langle\tau\rangle^{2}+\langle z\rangle^{2}}\right)^{1+\varepsilon}\right] \\
|\nabla \nabla C(x)| & \leq \frac{\text { const }}{|x|+1}\left[\frac{1}{\left(|| x^{0}|-| \mathbf{x}||+1\right)^{1+\varepsilon}}+\frac{1}{(|x|+1)^{\frac{1}{2}}\left(\left\|x^{0}|-| \mathbf{x}\right\|+1\right)^{\frac{1}{2}}}\right] \\
& \leq \frac{\text { const }}{\langle\tau\rangle\langle z\rangle}\left[\frac{1}{\langle\tau\rangle^{\frac{1}{2}}\langle z\rangle^{\frac{1}{2}}}\left(\frac{\langle\tau\rangle\langle z\rangle}{\langle\tau\rangle^{2}+\langle z\rangle^{2}}\right)^{\frac{1}{2}}+\left(\frac{\langle\tau\rangle\langle z\rangle}{\langle\tau\rangle^{2}+\langle z\rangle^{2}}\right)^{1+\varepsilon}\right]
\end{aligned}
$$




$$
\begin{aligned}
& \left.\begin{array}{l}
|(x \cdot \nabla+1) A(x)| \\
|\nabla(x \cdot \nabla C(x))|
\end{array}\right\} \leq \frac{\text { const }}{(|x|+1)\left(|| x^{0}|-| \mathbf{x}||+1\right)^{\varepsilon}} \\
& \leq \frac{\text { const }}{\langle\tau\rangle\langle z\rangle}\left(\frac{\langle\tau\rangle\langle z\rangle}{\langle\tau\rangle^{2}+\langle z\rangle^{2}}\right)^{\varepsilon}, \\
& \left|(x \cdot \nabla)^{k} C(x)\right| \leq \frac{\text { const }}{(|x|+1)^{\varepsilon}} \leq \frac{\text { const }}{\langle\tau\rangle^{\varepsilon}\langle z\rangle^{\varepsilon}}, \quad k=1,2, \\
& \left|x^{a} F_{a b}(x)\right| \leq \frac{\text { const }}{|x|+1} \leq \frac{\text { const }}{\langle\tau\rangle\langle z\rangle} .
\end{aligned}
$$

Proof. To prove the estimates on $A$ and $C$ in the half-space $x^{0} \geq 0$ it is sufficient to add the estimates of $A_{\text {out }}$ and $C_{\text {out }}$, as given by Theorem 21 , to the estimates of $A_{\mathrm{adv}}$ and $C_{\mathrm{adv}}$ following from the assumptions on $J$ and Lemma 20 (i) and (ii). For $x^{0} \geq 0$ one has

$$
\begin{aligned}
\left|\nabla^{\alpha} A_{\mathrm{adv}}(x)\right| & \leq \frac{\text { const }}{(|x|+1)^{|\alpha|+1}}, \quad|\alpha| \leq 3 \\
\left|\nabla^{\alpha}(x \cdot \nabla+1) A_{\mathrm{adv}}(x)\right| & =\left|\int D_{\mathrm{adv}}(x-y)(y \cdot \nabla+3+|\alpha|) \nabla^{\alpha} J(y) d y\right| \\
& \leq \frac{\mathrm{const}}{(|x|+1)^{1+|\alpha|+\varepsilon}}, \quad|\alpha| \leq 1 .
\end{aligned}
$$

Moreover,

$$
\begin{aligned}
& x \cdot \nabla C_{\mathrm{adv}}(x)=x^{c}(x \cdot \nabla+1) A_{\mathrm{adv} c}(x), \\
& (x \cdot \nabla)^{2} C_{\mathrm{adv}}(x)=x \cdot \nabla C_{\mathrm{adv}}(x)+x^{c} x^{d} \nabla_{d}(x \cdot \nabla+1) A_{\mathrm{adv} c}(x), \\
& x^{a} F_{a b}(x)=(x \cdot \nabla+1) A_{b}(x)-\nabla_{b} C(x) .
\end{aligned}
$$

and all the estimates in the $x$-form now easily follow. Similarly for $x^{0} \leq 0$ with the use of $A_{\text {in }}$ and $A_{\text {ret }}$. The $(\tau, \mathbf{z})$-form of the estimates is the consequence of Lemma 16.

\section{Appendix I. Special Gauge in Special Variables}

We consider now our field in the special gauge (73), which we write in the form

$$
\mathcal{A}=A-\nabla S_{\ell}, \quad S_{\ell}=\log (\langle\tau\rangle\langle z\rangle) C, \quad C(x)=x \cdot A .
$$

We need the components of this potential, and various expressions involving them, in our special coordinates system. For the sake of this section we introduce the abbreviation

$$
\ell_{\tau z}=\log (\langle\tau\rangle\langle z\rangle)
$$

Theorem 23. The special gauge potential $\hat{\mathcal{A}}_{\mu}(\tau, \mathbf{z})$ is a $C^{2}$ field, and the following estimates are satisfied:

$$
\begin{aligned}
& \left|\hat{F}_{i \tau}\right| \leq \frac{\text { const }}{\langle\tau\rangle\langle z\rangle}, \quad\left|z^{i} \hat{F}_{i \tau}\right| \leq \frac{\text { const }}{\langle\tau\rangle}, \quad\left|\hat{F}_{i j}\right| \leq \text { const }, \quad\left|z^{i} \hat{F}_{i j}\right| \leq \frac{\text { const }}{\langle z\rangle}, \\
& \left|\hat{\mathcal{A}}_{\tau}\right| \leq \text { const }\left[\frac{1+\log \langle\tau\rangle}{\langle\tau\rangle^{1+\varepsilon}}+\frac{\log \langle z\rangle}{\langle\tau\rangle^{3}}\right]
\end{aligned}
$$




$$
\begin{aligned}
& \left|\hat{\mathcal{A}}_{i}\right| \leq \operatorname{const} \frac{1+\log (\langle\tau\rangle\langle z\rangle)}{\langle z\rangle}, \quad\left|z^{i} \hat{\mathcal{A}}_{i}\right| \leq \operatorname{const} \frac{1+\log (\langle\tau\rangle\langle z\rangle)}{\langle z\rangle^{\varepsilon}} \\
& \left|\partial_{i} \hat{\mathcal{A}}_{\tau}\right|+\left|z^{i} \partial_{i} \hat{\mathcal{A}}_{\tau}\right| \leq \mathrm{const} \frac{1+\log \langle\tau\rangle}{\langle\tau\rangle^{1+\varepsilon}} \\
& \left|\partial_{\tau} \hat{\mathcal{A}}_{i}\right|+\left|z^{i} \partial_{\tau} \hat{\mathcal{A}}_{i}\right| \leq \frac{\mathrm{const}}{\langle\tau\rangle} \\
& \left|\partial_{i} \hat{\mathcal{A}}_{j}\right| \leq \operatorname{const}(1+\log \langle\tau\rangle) \\
& \left|z^{i} \partial_{i} \hat{\mathcal{A}}_{j}\right|+\left|z^{i} \partial_{j} \hat{\mathcal{A}}_{i}\right| \leq \operatorname{const} \frac{1+\log (\langle\tau\rangle\langle z\rangle)}{\langle z\rangle} \\
& \left|z^{i} z^{j} \partial_{i} \hat{\mathcal{A}}_{j}\right| \leq \operatorname{const} \frac{1+\log (\langle\tau\rangle\langle z\rangle)}{\langle z\rangle^{\varepsilon}}
\end{aligned}
$$

The proof below allows formulation of more restrictive, but at the same time more sophisticated and less transparent estimates. These given above are sufficient for our purposes.

Proof. According to Assumption III the potential $A$ is of class $C^{3}$, so $\mathcal{A}$ is of class $C^{2}$. For the calculation of the components and derivatives of $A$ and $C$, we use the following form of the coordinates change:

$$
\partial_{\tau} x=\frac{\tau}{\langle\tau\rangle^{2}} x+\frac{\langle z\rangle}{\langle\tau\rangle^{2}}(1,0), \quad \partial_{i} x=\left(\tau \frac{z^{i}}{\langle z\rangle},\langle\tau\rangle \delta^{i, .}\right),
$$

which leads further to additional identities

$$
\begin{aligned}
& z^{i} \partial_{i} x=x-\frac{\tau}{\langle z\rangle}(1,0), \\
& \partial_{i} \partial_{\tau} x=\frac{\tau}{\langle\tau\rangle^{2}} \partial_{i} x+\frac{z^{i}}{\langle\tau\rangle^{2}\langle z\rangle}(1,0), \quad \partial_{i} \partial_{j} x=\frac{\tau}{\langle z\rangle} d^{i j}(1,0),
\end{aligned}
$$

where $d^{i j}$ is given by

$$
d^{i j}=\delta^{i j}-\frac{z^{i} z^{j}}{\langle z\rangle^{2}}
$$

and some of its properties are

$$
\begin{aligned}
& \partial_{i}\left(\frac{z^{j}}{\langle z\rangle}\right)=\frac{d^{i j}}{\langle z\rangle}, \quad z^{i} d^{i j}=\frac{z^{j}}{\langle z\rangle^{2}}, \\
& \partial_{k} d^{i j}=\frac{-1}{\langle z\rangle^{2}}\left(z^{i} d^{j k}+z^{j} d^{i k}\right), \quad z^{i} \partial_{i} d^{j k}=-2 \frac{z^{j} z^{k}}{\langle z\rangle^{4}} .
\end{aligned}
$$

A straightforward calculation now gives

$$
\begin{aligned}
\hat{F}_{i \tau} & =\left(\partial_{i} x^{a}\right)\left(\partial_{\tau} x^{b}\right) F_{a b}=\left(\partial_{i} x^{a}\right)\left(\frac{\tau}{\langle\tau\rangle^{2}} F_{a b} x^{b}+\frac{\langle z\rangle}{\langle\tau\rangle^{2}} F_{a 0}\right), \\
z^{i} \hat{F}_{i \tau} & =\left(\frac{\langle z\rangle}{\langle\tau\rangle^{2}}+\frac{\tau^{2}}{\langle\tau\rangle^{2}\langle z\rangle}\right) x^{a} F_{a 0}, \\
\hat{F}_{i j} & =\left(\partial_{i} x^{a}\right)\left(\partial_{j} x^{b}\right) F_{a b}, \\
z^{i} \hat{F}_{i j} & =\left(x^{a} F_{a b}-\frac{\tau}{\langle z\rangle} F_{0 b}\right)\left(\partial_{j} x^{b}\right) .
\end{aligned}
$$




$$
\begin{aligned}
\hat{\mathcal{A}}_{\tau}= & \frac{\langle z\rangle}{\langle\tau\rangle^{2}} A_{0}-\ell_{\tau z} \partial_{\tau} C, \\
\hat{\mathcal{A}}_{i}= & \langle\tau\rangle d^{i j} A_{j}-\ell_{\tau z} \partial_{i} C, \\
z^{i} \hat{\mathcal{A}}_{i}= & \frac{\langle\tau\rangle}{\langle z\rangle^{2}} z^{i} A_{i}-\ell_{\tau z} z^{i} \partial_{i} C \\
\partial_{i} \hat{\mathcal{A}}_{\tau}= & \frac{z^{i}}{\langle\tau\rangle^{2}\langle z\rangle} A_{0}+\frac{\langle z\rangle}{\langle\tau\rangle^{2}} \partial_{i} A_{0}-\frac{z^{i}}{\langle z\rangle^{2}} \partial_{\tau} C-\ell_{\tau z} \partial_{i} \partial_{\tau} C, \\
z^{i} \partial_{i} \hat{\mathcal{A}}_{\tau}= & \frac{\mathbf{z}^{2}}{\langle\tau\rangle^{2}\langle z\rangle} A_{0}+\frac{\langle z\rangle}{\langle\tau\rangle^{2}} z^{i} \partial_{i} A_{0}-\frac{|\mathbf{z}|^{2}}{\langle z\rangle^{2}} \partial_{\tau} C-\ell_{\tau z} z^{i} \partial_{i} \partial_{\tau} C, \\
\partial_{i} \hat{\mathcal{A}}_{j}= & \langle\tau\rangle\left[\left(\partial_{i} d^{j k}\right) A_{k}+d^{j k} \partial_{i} A_{k}\right]-\frac{z^{i}}{\langle z\rangle^{2}} \partial_{j} C-\ell_{\tau z} \partial_{i} \partial_{j} C, \\
z^{i} \partial_{i} \hat{\mathcal{A}}_{j}= & \langle\tau\rangle\left[-2 \frac{z^{j} z^{k}}{\langle z\rangle^{4}} A_{k}+d^{j k} z^{i} \partial_{i} A_{k}\right]-\frac{|\mathbf{z}|^{2}}{\langle z\rangle^{2}} \partial_{j} C-\ell_{\tau z} z^{i} \partial_{i} \partial_{j} C, \\
z^{i} z^{j} \partial_{i} \hat{\mathcal{A}}_{j}= & \frac{\langle\tau\rangle}{\langle z\rangle^{2}}\left[-2 \frac{|\mathbf{z}|^{2}}{\langle z\rangle^{2}} z^{k} A_{k}+z^{i} z^{k} \partial_{i} A_{k}\right]-\frac{|\mathbf{z}|^{2}}{\langle z\rangle^{2}} z^{j} \partial_{j} C-\ell_{\tau z} z^{i} z^{j} \partial_{i} \partial_{j} C,
\end{aligned}
$$

For the estimation of all these field and potential expressions, we use Theorem 22. We note that

$$
\left|\partial_{i} x\right| \leq \operatorname{const}\langle\tau\rangle, \quad\left|\partial_{\tau} x\right| \leq \operatorname{const}\langle z\rangle, \quad\left|\partial_{z}^{\alpha} d^{i j}\right| \leq \frac{\text { const }}{\langle z\rangle^{|\alpha|}},|\alpha| \leq 2 .
$$

Moreover, when using the estimates (130), (131) and (132) we shall make use of the obvious inequalities

$$
\frac{\langle\tau\rangle\langle z\rangle}{\langle\tau\rangle^{2}+\langle z\rangle^{2}} \leq \min \left\{\frac{1}{2}, \frac{\langle\tau\rangle}{\langle z\rangle}, \frac{\langle z\rangle}{\langle\tau\rangle}\right\} \leq\left\{\begin{array}{l}
\langle\tau\rangle\langle z\rangle^{-1} \\
\langle\tau\rangle^{-1}\langle z\rangle \\
2^{-1}
\end{array}\right.
$$

(the choice of one of them will be dictated by a particular need). In this way we find

$$
\begin{aligned}
\left|\hat{F}_{i \tau}\right| & \leq \operatorname{const}\left(\left|F_{a b} x^{b}\right|+\frac{\langle z\rangle}{\langle\tau\rangle}\left|F_{a 0}\right|\right) \leq \frac{\text { const }}{\langle\tau\rangle\langle z\rangle} \\
\left|z^{i} \hat{F}_{i \tau}\right| & \leq \operatorname{const}\left(\frac{\langle z\rangle}{\langle\tau\rangle^{2}}+\frac{1}{\langle z\rangle}\right)\left|x^{a} F_{a 0}\right| \leq \frac{\text { const }}{\langle\tau\rangle}\left(\frac{1}{\langle\tau\rangle^{2}}+\frac{1}{\langle z\rangle^{2}}\right) \\
\left|\hat{F}_{i j}\right| & \leq \operatorname{const}\langle\tau\rangle^{2}\left|F_{a b}\right| \leq \text { const } \\
\left|z^{i} \hat{F}_{i j}\right| & \leq \operatorname{const}\left(\langle\tau\rangle\left|x^{a} F_{a b}\right|+\frac{\langle\tau\rangle^{2}}{\langle z\rangle}\left|F_{0 b}\right|\right) \leq \frac{\text { const }}{\langle z\rangle}
\end{aligned}
$$

which exhausts the claims on the field $F$.

The estimation of the potential expressions is more tedious, and we consider it in steps. In the first step we estimate the $\tau$ - and $\mathbf{z}$-dependent coefficients, which gives

$$
\left|\hat{\mathcal{A}}_{\tau}\right| \leq \frac{\langle z\rangle}{\langle\tau\rangle^{2}}\left|A_{0}\right|+\ell_{\tau z}\left|\partial_{\tau} C\right|
$$




$$
\begin{aligned}
& \left|\hat{\mathcal{A}}_{i}\right| \leq \operatorname{const}\langle\tau\rangle\left|A_{j}\right|+\ell_{\tau z}\left|\partial_{i} C\right|, \quad\left|z^{i} \hat{\mathcal{A}}_{i}\right| \leq \mathrm{const} \frac{\langle\tau\rangle}{\langle z\rangle}\left|A_{i}\right|+\ell_{\tau z}\left|z^{i} \partial_{i} C\right| \\
& \left|\partial_{i} \hat{\mathcal{A}}_{\tau}\right| \leq \frac{1}{\langle\tau\rangle^{2}}\left(\left|A_{0}\right|+\langle z\rangle\left|\partial_{i} A_{0}\right|\right)+\frac{1}{\langle z\rangle}\left|\partial_{\tau} C\right|+\ell_{\tau z}\left|\partial_{i} \partial_{\tau} C\right| \\
& \left|z^{i} \partial_{i} \hat{\mathcal{A}}_{\tau}\right| \leq \frac{\langle z\rangle}{\langle\tau\rangle^{2}}\left(\left|A_{0}\right|+\left|z^{i} \partial_{i} A_{0}\right|\right)+\left|\partial_{\tau} C\right|+\ell_{\tau z}\left|z^{i} \partial_{i} \partial_{\tau} C\right| \\
& \left|\partial_{i} \hat{\mathcal{A}}_{j}\right| \leq \operatorname{const}\langle\tau\rangle\left(\frac{1}{\langle z\rangle}\left|A_{k}\right|+\left|\partial_{i} A_{k}\right|\right)+\frac{1}{\langle z\rangle}\left|\partial_{j} C\right|+\ell_{\tau z}\left|\partial_{i} \partial_{j} C\right|, \\
& \left|z^{i} \partial_{i} \hat{\mathcal{A}}_{j}\right| \leq \operatorname{const}\langle\tau\rangle\left(\frac{1}{\langle z\rangle^{2}}\left|A_{k}\right|+\left|z^{i} \partial_{i} A_{k}\right|\right)+\left|\partial_{j} C\right|+\ell_{\tau z}\left|z^{i} \partial_{i} \partial_{j} C\right|, \\
& \left|z^{i} z^{j} \partial_{i} \hat{\mathcal{A}}_{j}\right| \leq \operatorname{const} \frac{\langle\tau\rangle}{\langle z\rangle}\left(\left|A_{k}\right|+\left|z^{i} \partial_{i} A_{k}\right|\right)+\left|z^{j} \partial_{j} C\right|+\ell_{\tau z}\left|z^{i} z^{j} \partial_{i} \partial_{j} C\right|,
\end{aligned}
$$

Next, we evaluate the $A$ - and $C$-terms:

$$
\begin{aligned}
& \partial_{i} A_{b}=\left(\partial_{i} x^{a}\right) \nabla_{a} A_{b}, \quad z^{i} \partial_{i} A_{b}=x \cdot \nabla A_{b}-\frac{\tau}{\langle z\rangle} \nabla_{0} A_{b}, \\
& \partial_{i} \partial_{j} A_{c}=\left(\partial_{i} x^{a}\right)\left(\partial_{j} x^{b}\right) \nabla_{a} \nabla_{b} A_{c}+\frac{\tau}{\langle z\rangle} d^{i j} \nabla_{0} A_{c}
\end{aligned}
$$

(the latter term is not needed for the present proof, but its estimate given below is used in the proof of Theorem 9 (iii) (b)),

$$
\begin{aligned}
& \partial_{\tau} C=\frac{\tau}{\langle\tau\rangle^{2}} x \cdot \nabla C+\frac{\langle z\rangle}{\langle\tau\rangle^{2}} \nabla_{0} C, \\
& \partial_{i} C=\left(\partial_{i} x^{a}\right) \nabla_{a} C, \quad z^{i} \partial_{i} C=x \cdot \nabla C-\frac{\tau}{\langle z\rangle} \nabla_{0} C, \\
& \partial_{i} \partial_{\tau} C=\left(\partial_{i} x^{a}\right)\left[\frac{\tau}{\langle\tau\rangle^{2}} \nabla_{a}(x \cdot \nabla C)+\frac{\langle z\rangle}{\langle\tau\rangle^{2}} \nabla_{a} \nabla_{0} C\right]+\frac{z^{i}}{\langle\tau\rangle^{2}\langle z\rangle} \nabla_{0} C, \\
& z^{i} \partial_{i} \partial_{\tau} C=\frac{\tau}{\langle\tau\rangle^{2}}\left[(x \cdot \nabla)^{2} C-\nabla_{0}^{2} C\right]+\left[\frac{\langle z\rangle}{\langle\tau\rangle^{2}}-\frac{\tau^{2}}{\langle\tau\rangle^{2}\langle z\rangle}\right] \nabla_{0}(x \cdot \nabla C) \\
& -\frac{1}{\langle\tau\rangle^{2}\langle z\rangle} \nabla_{0} C, \\
& \partial_{i} \partial_{j} C=\left(\partial_{i} x^{a}\right)\left(\partial_{j} x^{b}\right) \nabla_{a} \nabla_{b} C+\frac{\tau}{\langle z\rangle} d^{i j} \nabla_{0} C, \\
& z^{i} \partial_{i} \partial_{j} C=\left(\partial_{j} x^{a}\right)\left[\nabla_{a}(x \cdot \nabla-1) C-\frac{\tau}{\langle z\rangle} \nabla_{a} \nabla_{0} C\right]+\frac{\tau z^{j}}{\langle z\rangle^{3}} \nabla_{0} C, \\
& z^{i} z^{j} \partial_{i} \partial_{j} C=(x \cdot \nabla-1) x \cdot \nabla C-2 \frac{\tau}{\langle z\rangle} \nabla_{0}(x \cdot \nabla C)+\frac{\tau^{2}}{\langle z\rangle^{2}} \nabla_{0}^{2} C \\
& \quad+\frac{\tau}{\langle z\rangle}\left(2+\frac{|\mathbf{z}|^{2}}{\langle z\rangle^{2}}\right) \nabla_{0} C .
\end{aligned}
$$

Now we can estimate the $A$ - and $C$-terms:

$$
\left|A_{a}\right| \leq \frac{\text { const }}{\langle\tau\rangle\langle z\rangle}, \quad\left|\partial_{i} A_{b}\right| \leq \text { const } \min \left\{\frac{1}{\langle z\rangle}, \frac{1}{\langle\tau\rangle}\right\},
$$




$$
\begin{aligned}
& \left|z^{i} \partial_{i} A_{b}\right| \leq \frac{\text { const }}{\langle\tau\rangle\langle z\rangle}, \quad\left|\partial_{i} \partial_{j} A_{b}\right| \leq \text { const } \frac{\langle\tau\rangle}{\langle z\rangle}, \\
& \left|\partial_{\tau} C\right| \leq \operatorname{const}\left(\frac{1}{\langle\tau\rangle^{1+\varepsilon}\langle z\rangle^{\varepsilon}}+\frac{1}{\langle\tau\rangle^{3}}\right) \\
& \left|\partial_{i} C\right| \leq \frac{\text { const }}{\langle z\rangle}, \quad\left|z^{i} \partial_{i} C\right| \leq \frac{\text { const }}{\langle z\rangle^{\varepsilon}}, \\
& \left|\partial_{i} \partial_{\tau} C\right| \leq \frac{\text { const }}{\langle\tau\rangle^{1+\varepsilon}\langle z\rangle^{1-\varepsilon}}, \quad\left|z^{i} \partial_{i} \partial_{\tau} C\right| \leq \frac{\text { const }}{\langle\tau\rangle^{1+\varepsilon}\langle z\rangle^{\varepsilon}}, \\
& \left|\partial_{i} \partial_{j} C\right| \leq \text { const } \min \left\{1, \frac{\langle\tau\rangle}{\langle z\rangle}\right\}, \\
& \left|z^{i} \partial_{i} \partial_{j} C\right| \leq \frac{\text { const }}{\langle z\rangle}, \quad\left|z^{i} z^{j} \partial_{i} \partial_{j} C\right| \leq \frac{\text { const }}{\langle z\rangle^{\varepsilon}} .
\end{aligned}
$$

Substituting these estimates in the bounds on the potential expressions obtained earlier in the proof, one arrives at the estimates given for them in the thesis. The only less obvious estimation on the way is that of the term

$$
\log (\langle\tau\rangle\langle z\rangle) \min \left\{1, \frac{\langle\tau\rangle}{\langle z\rangle}\right\}
$$

appearing in $\left|\partial_{i} \hat{\mathcal{A}}_{j}\right|$. For $\langle z\rangle \leq\langle\tau\rangle$ this is bounded by $2 \log \langle\tau\rangle$, while for $\langle z\rangle \geq$ $\langle\tau\rangle$ we write $\log (\langle\tau\rangle\langle z\rangle)=2 \log \langle\tau\rangle+\log (\langle z\rangle /\langle\tau\rangle)$ and estimate the term by

$$
2 \log \langle\tau\rangle+\left(\frac{\langle z\rangle}{\langle\tau\rangle}\right)^{-1} \log \left(\frac{\langle z\rangle}{\langle\tau\rangle}\right) \leq \operatorname{const}(1+\log \langle\tau\rangle) \text {. }
$$

Finally, we write

$$
\begin{aligned}
& \left|\partial_{\tau} \hat{\mathcal{A}}_{i}\right| \leq\left|\partial_{i} \hat{\mathcal{A}}_{\tau}\right|+\left|\hat{F}_{i \tau}\right|, \quad\left|z^{i} \partial_{\tau} \hat{\mathcal{A}}_{i}\right| \leq\left|z^{i} \partial_{i} \hat{\mathcal{A}}_{\tau}\right|+\left|z^{i} \hat{F}_{i \tau}\right| \\
& \left|z^{j} \partial_{i} \hat{\mathcal{A}}_{j}\right| \leq\left|z^{j} \partial_{j} \hat{\mathcal{A}}_{i}\right|+\left|z^{j} \hat{F}_{i j}\right|
\end{aligned}
$$

to obtain the last missing estimates.

\section{References}

[1] Bär, C., Ginoux, N., Pfäffle, F.: Wave Equations on Lorentzian Manifolds and Quantization, ESI Lectures in Mathematics and Physics. EMS, Zurich (2007). arxiv:0806.1036 [math.DG]

[2] Dereziński, J., Gérard, Ch.: Scattering Theory of Classical and Quantum NParticle Systems. Springer, Berlin (1997)

[3] Dirac, P.A.M.: Gauge-invariant formulation of quantum electrodynamics. Can. J. Phys. 33, 650-660 (1955)

[4] Duch, P.: Infrared problem in perturbative quantum field theory. arXiv:1906.00940. [math-ph]

[5] Dybalski, W.: From Faddeev-Kulish to LSZ. Towards a non-perturbative description of colliding electrons. Nucl. Phys. B 925, 455-469 (2017)

[6] Dybalski, W., Wegener, B.: Asymptotic charges, large gauge transformations and inequivalence of different gauges in external current QED. JHEP 11, 126 (2019). $12 \mathrm{pp}$ 
[7] Flato, M., Simon, J., Taflin, E.: Asymptotic completeness, global existence and the infrared problem for the Maxwell-Dirac equations. arXiv:hep-th/9502061v1 (1995). Asymptotic Completeness, Global Existence and the Infrared Problem for the Maxwell-Dirac Equations, Memoirs of the American Mathematical Society (Book 606), AMS, 1997

[8] Herdegen, A.: Long-range effects in asymptotic fields and angular momentum of classical field electrodynamics. J. Math. Phys. 36, 4044-4086 (1995)

[9] Herdegen, A.: Semidirect product of CCR and CAR algebras and asymptotic states in quantum electrodynamics. J. Math. Phys. 39, 1788-1817 (1998)

[10] Herdegen, A.: Asymptotic structure of electrodynamics revisited. Lett. Math. Phys. 107, 1439-1470 (2017)

[11] Herdegen, A.: Electromagnetic gauge choice for scattering of Schrödinger particle. Ann. H. Poincaré 20, 3633-3650 (2019)

[12] Marecki, P.: Electron scattering in long-range electromagnetic field, MSc Thesis, Jagiellonian University, Kraków (2000). (unpublished)

[13] Morchio, G. and Strocchi, F.: The infrared problem in QED: A lesson from a model with Coulomb interaction and realistic photon emission, Ann. H. Poincaré 17, 2699-2739 (2016). arXiv: 1410.7289v3 [hep-th]

[14] Reed, M., Simon, B.: Methods of Modern Mathematical Physics, vol. I. Academic Press, San Diego (1975)

[15] Reed, M., Simon, B.: Methods of Modern Mathematical Physics, vol. II. Academic Press, San Diego (1979)

[16] Ruijsenaars, S.N.M.: Charged particles in external fields. I. Classical theory. J. Math. Phys. 18, 720-737 (1977)

[17] Scharf, G.: Finite Quantum Electrodynamics. The Causal Approach, 2nd edn. Springer, Berlin (1995)

[18] Steinmann, O.: Perturbative Quantum Electrodynamics and Axiomatic Field Theory. Springer, Berlin (2000)

[19] Vainberg, B.R.: Asymptotic Methods in Equations of Mathematical Physics. Gordon and Breach, New York (1989)

Andrzej Herdegen

Institute of Theoretical Physics

Jagiellonian University

ul. S. Łojasiewicza 11

30-348 Kraków

Poland

e-mail: herdegen@th.if.uj.edu.pl

Communicated by Jan Derezinski.

Received: July 6, 2020.

Accepted: December 30, 2020. 